\title{
Space Weather: The Solar Perspective
}

\author{
Rainer Schwenn \\ Max-Planck-Institut für Sonnenystemforschung, \\ D-37191 Katlenburg-Lindau, \\ Germany \\ email: schwenn@mps.mpg.de
}

Accepted on 10 July 2006

Published on 9 August 2006

(Revised on 16 June 2010)

\begin{abstract}
The term space weather refers to conditions on the Sun and in the solar wind, magnetosphere, ionosphere, and thermosphere that can influence the performance and reliability of space-borne and ground-based technological systems and that can affect human life and health. Our modern hi-tech society has become increasingly vulnerable to disturbances from outside the Earth system, in particular to those initiated by explosive events on the Sun: Flares release flashes of radiation that can heat up the terrestrial atmosphere such that satellites are slowed down and drop into lower orbits, solar energetic particles accelerated to near-relativistic energies may endanger astronauts traveling through interplanetary space, and coronal mass ejections are gigantic clouds of ionized gas ejected into interplanetary space that after a few hours or days may hit the Earth and cause geomagnetic storms. In this review, I describe the several chains of actions originating in our parent star, the Sun, that affect Earth, with particular attention to the solar phenomena and the subsequent effects in interplanetary space.
\end{abstract}

This review is licensed under a Creative Commons Attribution-Non-Commercial-NoDerivs 3.0 Germany License. http://creativecommons.org/licenses/by-nc-nd/3.0/de/ 


\section{Imprint / Terms of Use}

Living Reviews in Solar Physics is a peer reviewed open access journal published by the Max Planck Institute for Solar System Research, Max-Planck-Str. 2, 37191 Katlenburg-Lindau, Germany. ISSN $1614-4961$.

This review is licensed under a Creative Commons Attribution-Non-Commercial-NoDerivs 3.0 Germany License: http://creativecommons.org/licenses/by-nc-nd/3.0/de/

Because a Living Reviews article can evolve over time, we recommend to cite the article as follows:

Rainer Schwenn,

"Space Weather: The Solar Perspective",

Living Rev. Solar Phys., 3, (2006), 2. [Online Article]: cited [<date>], http://www.livingreviews.org/lrsp-2006-2

The date given as $<$ date $>$ then uniquely identifies the version of the article you are referring to. 


\section{Article Revisions}

Living Reviews supports two ways of keeping its articles up-to-date:

Fast-track revision A fast-track revision provides the author with the opportunity to add short notices of current research results, trends and developments, or important publications to the article. A fast-track revision is refereed by the responsible subject editor. If an article has undergone a fast-track revision, a summary of changes will be listed here.

Major update A major update will include substantial changes and additions and is subject to full external refereeing. It is published with a new publication number.

For detailed documentation of an article's evolution, please refer to the history document of the article's online version at http://www. livingreviews.org/lrsp-2006-2.

16 June 2010: Updated this review on status of planned and launched missions. Added references to three related Living Reviews articles and to the LASCO CME catalog (Robbrecht et al., 2009).

Page 5: Updated reference to Haigh (2007).

Page 5: Added Hinode and SDO.

Page 9: Updated reference to Pulkkinen (2007).

Page 21: Added reference to Benz (2008).

Page 22: Added links to RHESSI, Hinode, and Wikipedia. Removed original link to YOHKOH (http://solar.physics.montana.edu/sxt/).

Page 22: Updated link to HASTA.

Page 24: Corrected year from 1959 to 1859.

Page 40: Added new reference to the LASCO CME catalog (Robbrecht et al., 2009.)

Page 53: Updated status of missions. 


\section{Contents}

1 Introduction $\quad 5$

2 The Solar Wind as Shaper of the Earth's Environment 10

2.1 The solar wind as a two-state phenomenon . . . . . . . . . . . . . . . . 10

2.2 Solar wind in three dimensions . . . . . . . . . . . . . . . . . . . . 13

2.3 Solar wind and space weather . . . . . . . . . . . . . . . . 17

3 Radiation from Solar Flares $\quad 21$

3.1 Some historical remarks . . . . . . . . . . . . . . . . . . . . . . 21

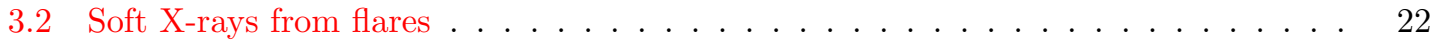

3.3 EUV and visible light . . . . . . . . . . . . . . . . . . . . 22

3.4 Hard X-rays . . . . . . . . . . . . . . . . . . . . . . . . . . . . . . . . . . . . . . . . . . . .

3.5 Impulsive microwave bursts . . . . . . . . . . . . . . . . . . . . . . . . . 23

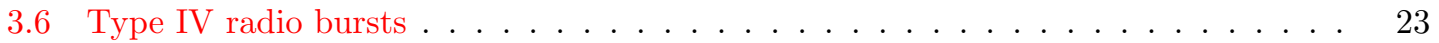

3.7 Gamma-rays (and white light) . . . . . . . . . . . . . . . . . 24

3.8 Type III radio bursts . . . . . . . . . . . . . . . . . . . . . . . . . . . . 24

3.9 Metric type II radio bursts . . . . . . . . . . . . . . . . . . . . . . . . . . . . . . . . . . . . . . . . . . . .

3.10 Kilometric type II radio bursts . . . . . . . . . . . . . . . . . . . . . . . . . . 29

3.11 Solar flares and space weather . . . . . . . . . . . . . . . . . . 30

4 Solar Energetic Particles (SEPs) $\quad 32$

4.1 SEPs: protons and other ions . . . . . . . . . . . . . . . . . . . . . . . . . . . . . . . .

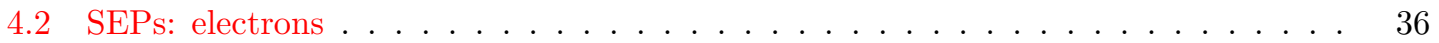

4.3 SEPs: neutrons . . . . . . . . . . . . . . . . . . . . 37

4.4 The acceleration of SEPs . . . . . . . . . . . . . . . . . . . . . . . . . . . . . . . . . . . . . . . . . . .

4.5 SEPs and space weather . . . . . . . . . . . . . . . . . . . 38

5 Coronal Mass Ejections (CMEs) 39

5.1 CME properties . . . . . . . . . . . . . . . . . . . . . . 39

5.2 Interplanetary counterparts of CMEs: ICMEs . . . . . . . . . . . . . . . . 47

5.3 CMEs, ICMEs, and space weather . . . . . . . . . . . . . . . 48

6 Concluding Remarks $\quad 53$

$\begin{array}{ll}\text { References } & 54\end{array}$

\section{List of Tables}

1 Average solar wind parameters at 1 AU, for the time around solar activity minimum. 13 


\section{Introduction}

The term space weather refers to conditions on the Sun and in the solar wind, magnetosphere, ionosphere, and thermosphere that can influence the performance and reliability of space-borne and ground-based technological systems and that can affect human life and health (definition used by the U.S. National Space Weather Plan). Of course, this definition also encompasses the generous energy supply from the Sun through its radiation that allows the existence of life on the Earth. However, this article is not meant to address this particular topic, except for the variability of radiation effects on very short time scales, e.g., in flares. Longer time scales such as decades or even centuries are covered in Living Reviews in Solar Physics by the article "The Sun and the Earth's Climate" by Haigh (2007).

Our modern hi-tech society has become increasingly vulnerable to disturbances from outside the Earth system, in particular to those initiated by explosive events on the Sun:

1. Flares release flashes of radiation covering an immense wavelength range (from radio waves to Gamma-rays) that can, e.g., heat up the terrestrial atmosphere within minutes such that satellites drop into lower orbits.

2. Solar energetic particles (SEPs), accelerated to near-relativistic energies during major solar storms arrive at the Earth's orbit within minutes and may, among other things, severely endanger astronauts traveling through interplanetary space, i.e., outside the Earth's protective magnetosphere.

3. Coronal mass ejections (CMEs), ejected into interplanetary space as gigantic clouds of ionized gas, that after a few hours or days may eventually hit the Earth and cause, among other effects, geomagnetic storms.

The economic consequences of these effects are enormous (see, e.g., Siscoe, 2000; Lanzerotti, 2001; Baker, 2004, see also further articles in the books by Song et al., 2001, and Daglis et al., 2004). That's one reason why space weather and its predictability have recently attained major attention, not only with the involved scientists but also with the general public. Another reason is the new quality of observational data that have been obtained over the last decade from a new generation of space-based instruments. A huge fleet of spacecraft (ULYSSES, SOHO, YOKHOH, WIND, ACE, TRACE, RHESSI, Hinode, SDO) has allowed us to advance our understanding of the processes involved near the Sun, in interplanetary space, and in the near-Earth environment, and thus to renew our picture of the Sun, the heliosphere, and the solar-terrestrial relationships (see, e.g., the review by Crooker, 2000).

For setting the stage for this article, I present a series of observations of the famous "Halloween events" that occurred during several days in late October/early November 2003. All important aspects of the space weather issue are addressed here in a very impressive way. The animation in Figure 1 shows a sequence of images taken with the EIT telescope on SOHO. A few very active regions moved across the Earth-facing side of the Sun and produced several bright flares and massive eruptions. Some of them resulted in powerful CMEs (see Figure 2 and 3 which are series of coronagraph images taken by the LASCO C2 and C3 instruments on SOHO), that were pointed towards the Earth and caused major geomagnetic storms. This type of CME where the brightening occurs simultaneously all around the coronagraphs occulting disk are called halo CMEs (Howard et al., 1982).

Intense fluxes of SEPs with relativistic energies were also generated, capable enough to penetrate the skins of spacecraft and instruments and even damage some. The "snow showers" in the images of Figures 1, 2 and 3 were in fact caused by such particles. Fortunately, the CCD cameras in these telescopes recovered after some hours. When it was finally realized how high that the radiation dose from such giant events can actually be, this issue became a primary concern in 


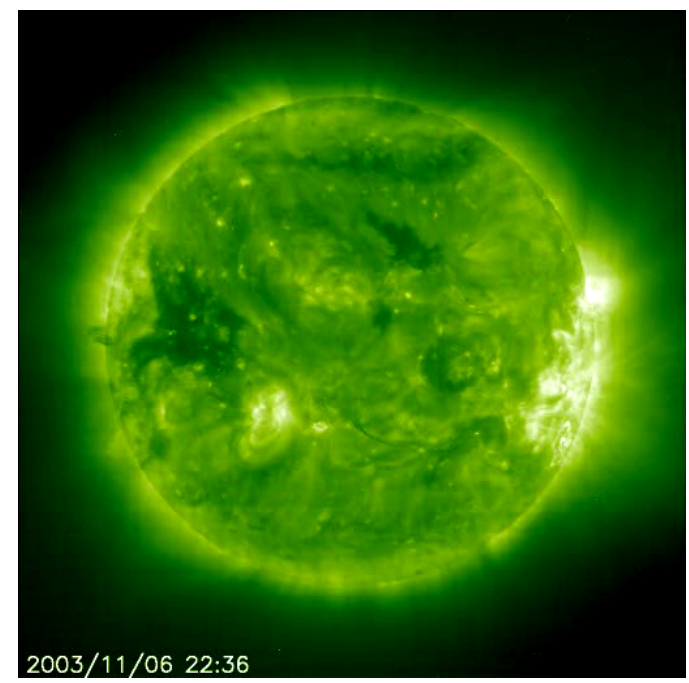

Figure 1: Still from a movie showing A sequence of images taken with EIT on SOHO in the light of the $19.5 \mathrm{~nm}$ line, between October 27 and November 7, 2003. Several active regions associated with big sunspots released a series of major flares and CMEs: the "Halloween events". The "snowstorms" following the major eruptions were caused by relativistic protons from the flare, that reached the Earth within minutes and were able to penetrate the spacecraft and instrument housings. (To watch the movie, please go to the online version of this review article at http://www.livingreviews.org/lrsp-2006-2.)

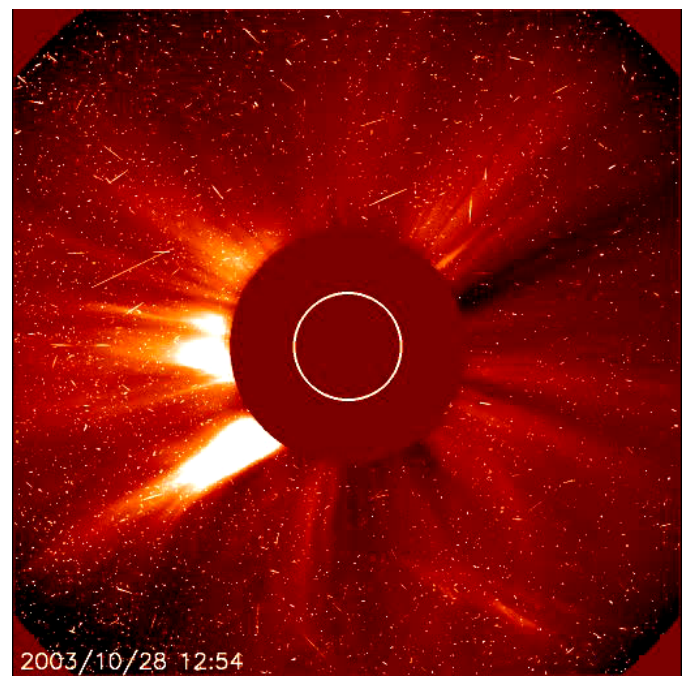

Figure 2: Still from a movie showing A sequence of white-light images taken with the coronagraph LASCO C2 on SOHO, between October 27 and 31, 2003. Several halo CMEs occurred, but they are hard to recognize because of the violent "snowstorms" from relativistic SEPs. Note the little Kreutz comet plunging into the Sun on October 28, just hours before the first major eruption. (To watch the movie, please go to the online version of this review article at http://www.livingreviews.org/lrsp-2006-2.)

Living Reviews in Solar Physics

http://www. livingreviews.org/lrsp-2006-2 


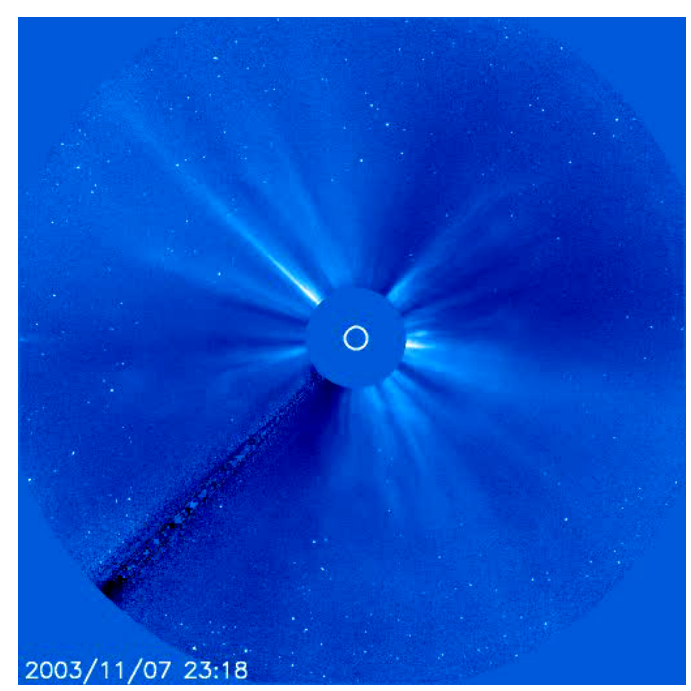

Figure 3: Still from a movie showing A similar sequence, taken with the coronagraph LASCO C3 on SOHO, between October 18 and November 2003. (To watch the movie, please go to the online version of this review article at http://www.livingreviews.org/lrsp-2006-2.)

manned space exploration. Adequate protective measures must be found to ensure the astronauts' safety on their future journeys to Moon and Mars (see, e.g., Wilson et al., 2004, and references therein).

The Halloween series of X-ray flares, SEP fluxes, interplanetary magnetic field (IMF) and geomagnetic index variations is illustrated in Figure 4. The X28 flare on November 4 was in fact the strongest solar X-ray flare since the beginning of regular recordings in 1968. The X-ray sensors on the GOES satellites even went into saturation. After some proper reconstruction using calibrated proxy data the real magnitude of this flare was determined X40 which means a peak flux of $4 \mathrm{~mW} / \mathrm{m}^{2}$ at Earth (Woods et al., 2004; Brodrick et al., 2005). Further, on October 30, two of the 12 strongest geomagnetic storms (compare Cliver and Svalgaard, 2004) since the beginning of $D_{\text {st }}$ recording in 1932 occurred $(-363 \mathrm{nT}$ and $-401 \mathrm{nT})$, with most dramatic consequences all over the globe. These storms were set loose right at those moments when the IMF turned southward (strong $B_{\mathrm{z}}$ south components).

Detailed analyses of these extraordinary Halloween events and their effects were assembled in special editions of Geophysical Research Letters and Journal of Geophysical Research (see http: //www.agu.org/journals/ss/VIOLCONN1/ and were reviewed by Veselovsky et al. (2004), (see also Gopalswamy et al., 2005b). These events demonstrate most impressively what space weather is about, with respect to both: its origin at the Sun, and its various effects on the Earth system.

Forecasting space weather effects is still a major challenge (Singer et al., 2001; Schwenn et al., 2005). The trustworthiness and accuracy in forecasting even the big solar events, i.e., flares and CMEs, and their impacts are still poor. They occur rather spontaneously, and we have not yet identified unique signatures that would indicate an imminent explosion and its probable onset time, location, strength, and significance for the Earth. The underlying physics is not sufficiently well understood, and thus we do not have appropriate warning tools at hands.

In this review, I will describe the several chains of actions originating in our parent star, the Sun, that affect Earth, with particular attention to the solar phenomena and the subsequent effects in interplanetary space. At first, we will inspect the solar wind itself: it is the medium in which the Earth system is imbedded and which determines the "ground state" of space weather. The solar wind interacts with the Earth's intrinsic magnetic field and thus shapes the magnetosphere. 


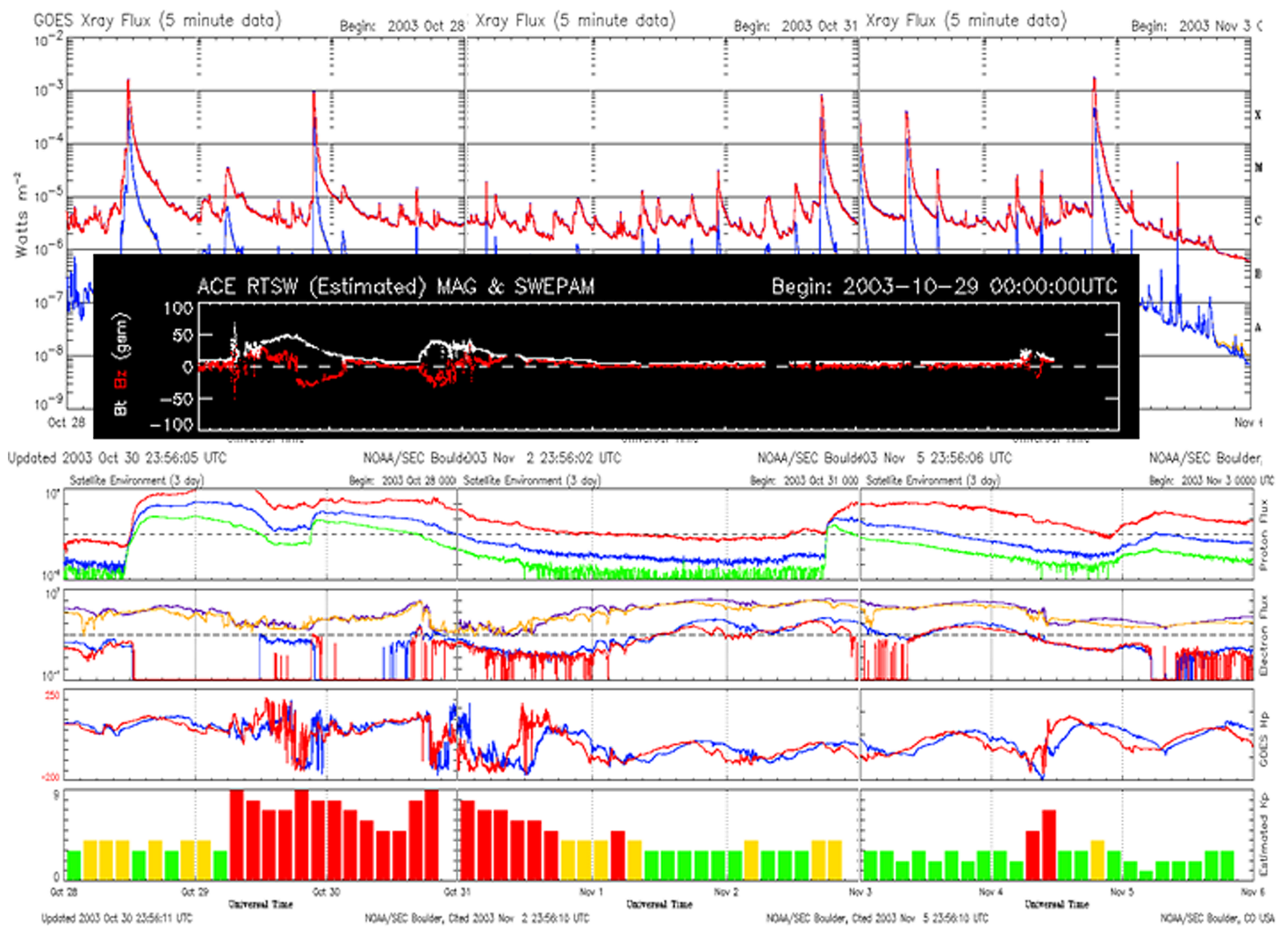

Figure 4: The Halloween series (from October 28 to November 6, 2003) of X-ray flares (upper 2 panels), interplanetary magnetic field (inserted panel; the red curve is the $B_{\mathrm{z}}$ component), SEP fluxes (next 3 panels), and geomagnetic index (Kp) variations (bottom panel). The data were assembled from the NOAA webpages starting from http://www.sec.noaa.gov/today.html and the ACE page at http://sec.noaa. gov/ace/ACErtsw_home.html. 
By its variability the solar wind constantly moulds and remodels the magnetosphere. Finally, the solar wind is the medium through which disturbances from the Sun have to propagate.

Once a disturbance has reached the outer boundaries of the Earth system, a whole new series of processes will be triggered that are controlled by the Earth's magnetic field, its ionosphere and atmosphere. Living Reviews in Solar Physics covers this issue in the article "Space Weather: Terrestrial Perspective" by Pulkkinen (2007). 


\section{The Solar Wind as Shaper of the Earth's Environment}

Space between the Sun and its planets is not empty as had been generally thought until the 1950s. It is filled by a tenuous magnetized plasma, which is a mixture of ions and electrons flowing away from the Sun: the solar wind. In fact, the Sun's outer atmosphere is so hot that not even the Sun's enormous gravity can prevent it from continually evaporating. The escaping plasma carries the solar magnetic field along, out to the border of the heliosphere where its dominance finally ends.

The solar wind (and the IMF carried with it) proves to be one key link between the solar atmosphere and the Earth system. Although the energy transferred by the solar wind is minuscule compared to both sunlight and those energies involved in Earth's atmosphere, the solar wind is capable of pin-pricking the Earth system which eventually may react in a highly nonlinear way. There are indications of effects reaching down as far as the troposphere, and our increasingly sophisticated high-tech civilization can indeed notice them and does, at times, even suffer from them. That is why the role of the Sun and the solar wind as the drivers of space weather have gained particular attention in the recent past.

Generally, the solar wind flow is diverted around Earth by its magnetosphere that is maintained by the Earth's intrinsic magnetic field. Solar wind particles cannot enter, unless there occurs a process called magnetic reconnection of interplanetary and planetary magnetic field lines. That may happen if the northward pointing Earth field on the front of the magnetosphere is hit by solar wind carrying a southward pointing interplanetary field. In such case, significant geomagnetic disturbances of various kinds are initiated (see, e.g., Tsurutani et al., 1988). Note that usually the IMF near the Earth does not have northward or southward pointing components. It is the intention of this section to describe the various effects by which the IMF can be tilted such that major $B_{\mathrm{z}}$ south excursions actually do occur.

The status of knowledge on the solar wind before 1972 had been very well summarized in the textbook by Hundhausen (1972). Then, from the mid 1970s on, a new class of space missions (Skylab, Helios, Voyager, and Ulysses) equipped with a new generation of instruments had initiated a new epoch in solar and heliospheric research. Numerous important discoveries were made and are documented in the literature. Major reviews can be found, e.g., in Zirker (1977); Schwenn and Marsch (1990, 1991); Kohl and Cranmer (1999); Srivastava and Schwenn (2000); Balogh et al. (2001). A comparable step forward occurred in the mid 1990s when the Yohkoh, SOHO, WIND, ACE, and TRACE spacecraft went into operation. Reviews can readily be found, e.g., in the series of SOHO Workshop Proceedings published in the ESA SP series. I further recommend the reviews in Living Reviews in Solar Physics "Kinetic Physics of the Solar Corona and Solar Wind" by Marsch (2006) and "The Solar Wind as a Turbulence Laboratory" by Bruno and Carbone (2005) and the article by Marsch et al. (2003).

\subsection{The solar wind as a two-state phenomenon}

From eclipse observations it has been well known that the solar corona is highly structured and dynamic (Figure 5). It changes its shape enormously during the solar activity cycle. Hence, it was no great surprise when both these properties (spatial structure and temporal variability) were found to be reproduced in the corona's offspring, i.e., the solar wind.

It was not until the Skylab era in 1973/74 when so-called coronal holes were discovered to be the sources of long-lived solar wind high-speed streams (Krieger et al., 1973). Coronal holes are usually located above inactive parts of the Sun, where "open" magnetic field lines prevail, e.g., at the polar caps around activity minima. In contrast, the more active near-equatorial regions on the Sun are most often associated with closed magnetic structures, such as bipolar loop systems and helmet streamers on top. From here, the more turbulent slow solar wind emerges. The embedded density fluctuations allow visualization of this type of solar wind. The images of Figure 6 (taken

Living Reviews in Solar Physics

http://www. livingreviews.org/lrsp-2006-2 


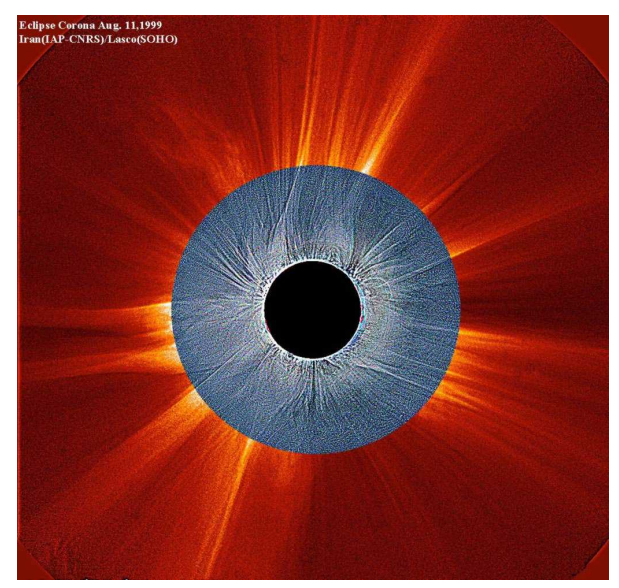

Figure 5: Eclipse image of August 11, 1999, combined with a simultaneously taken coronagraph image (LASCO $\mathrm{C} 2$ on $\mathrm{SOHO}$ ). For both images, the contrast was artificially enhanced in order to reveal the large-scale coronal structures and their sources in the lower corona (color print courtesy of S. Koutchmy, description in Koutchmy et al., 2004).

with the most sensible coronagraph LASCO $\mathrm{C} 3$ on $\mathrm{SOHO}$ ) reveal that it is ejected continually near the equatorial plane, with the appearance of cigarette smoke. Sometimes the patterns in the out-flowing clouds can be tracked like "leaves in the wind" such that Sheeley Jr et al. (1997) could even determine the speed profile. Unfortunately, the high-speed streams above the poles remain invisible, they do not have sufficiently strong density fluctuations.

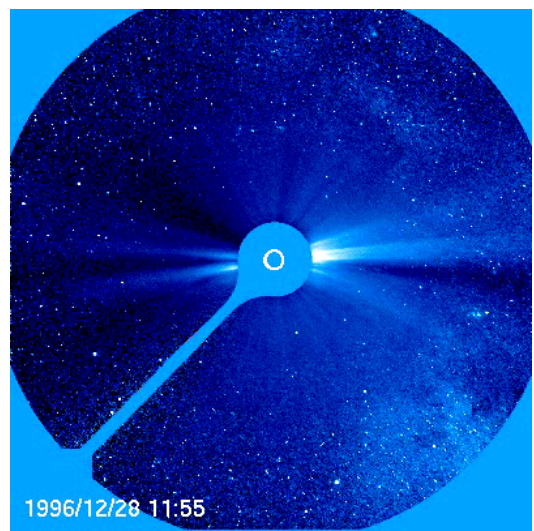

Figure 6: Still from a movie showing A sequence of white-light images taken with the coronagraph LASCO C3 on SOHO, between December 22 and 28, 1996. The Sun is at its minimum of activity. The density fluctuations in the slow solar wind near the equatorial plane makes this type of solar wind visible. Note the passage of the (occulted) Sun through the milky way around Christmas time. Note further the little Kreutz comet plunging into the Sun on December 22. (To watch the movie, please go to the online version of this review article at http://www.livingreviews.org/lrsp-2006-2.)

It is important to note that both: the coronal holes as well as their offspring, the high-speed solar wind streams are representatives of the inactive or "quiet" Sun. Thus, the only state of the solar wind that may deserve the label "quiet" is the high-speed wind, rather than the more variable slow wind from above active regions. This perception, first described by Feldman et al. (1976) and Bame et al. (1977), caused a major paradigm change. No longer could the slow wind be considered 
the "quiet" or "ground state" type although it would fit much better to the famous model of a thermally driven solar wind as derived by Parker (1958).

Figure 7 exhibits the two states of the near-minimum corona in 1998 and the associated solar wind streams rather nicely. The parts of this combined image were taken almost simultaneously from the EIT and LASCO telescopes on SOHO. This figure shows the two states by their different brightness in the corona. Note also how well separated from each other they are, both in the low corona as well as in the extended corona, i.e., in the solar wind.

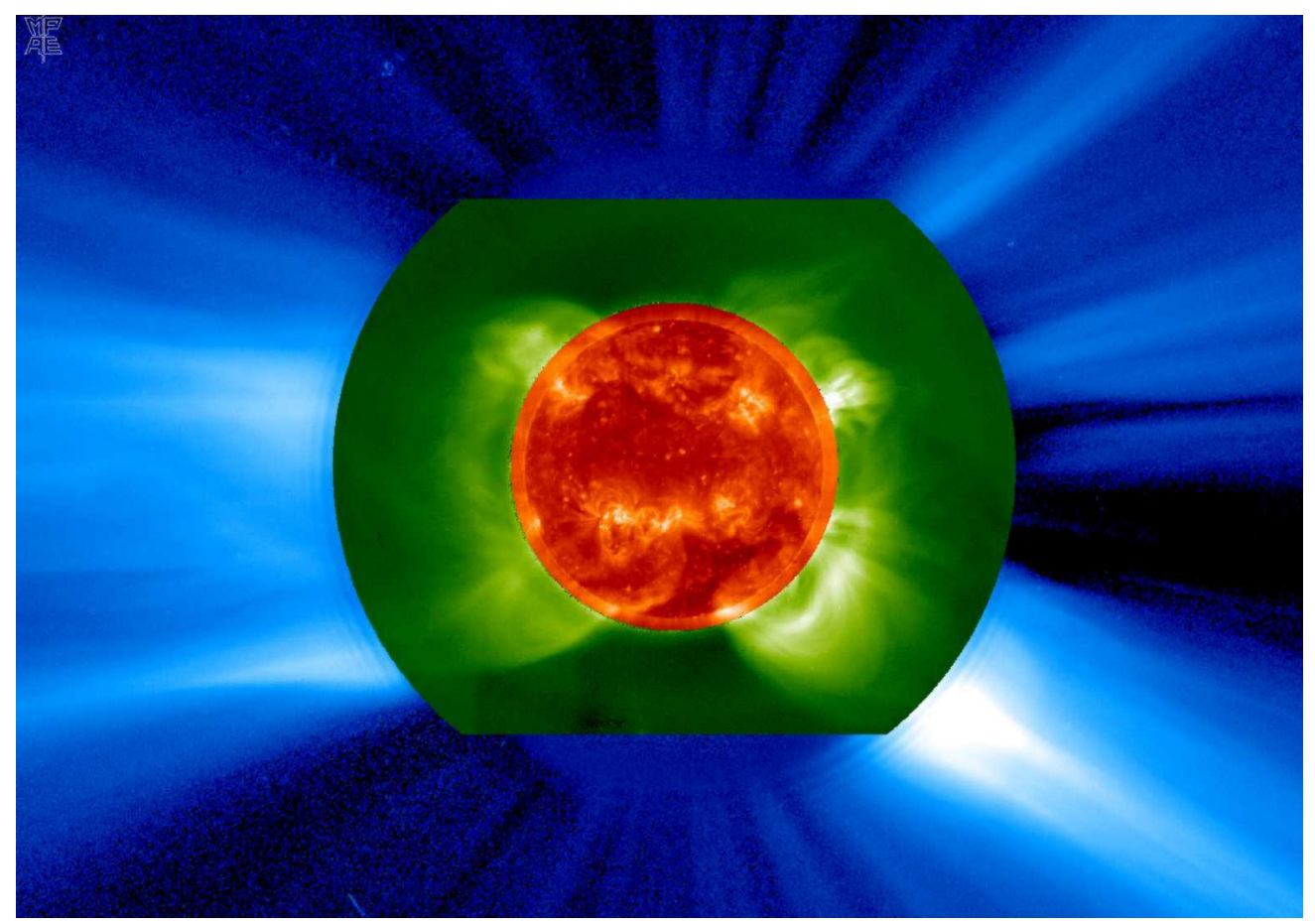

Figure 7: This figure shows the two states by their different brightness in the corona. Note also how well separated from each other they are, both in the low corona as well as in the extended corona, i.e., in the solar wind.

In fact, the existence of sharp boundaries between solar wind streams (in longitude as well as in latitude) had already been noticed by Rosenbauer et al. (1977) and Schwenn et al. (1978) on the basis of in situ measurements from the Helios solar probes that went as close as 0.3 AU to the Sun. These two basic types of quasi-steady solar wind differ markedly in their main properties and by the location and magnetic topology of their sources in the corona, thus probably in their acceleration mechanism.

The main characteristics of the two basic states as determined by Schwenn (1990) from measurements in the plane of the ecliptic are listed in Table 1.

The main differences between the two states are, apart from the speed itself, the particle densities, the mass flux, and the helium content. However, note the striking similarities in the flux densities of energy and momentum. The latter is equivalent to the ram pressure the solar wind is exerting on an obstacle like the Earth. It appears to be invariant with the flow state within $7 \%$. The same result was found to apply for the latitudinal stream structure up to at least $30^{\circ}$ on heliographic latitude (Bruno et al., 1986). That means that size and shape of the Earth's magnetosphere are not affected much by the solar wind stream structure. That aspect should be kept in mind in the space weather context.

Living Reviews in Solar Physics

http://www. livingreviews.org/lrsp-2006-2 
Table 1: Average solar wind parameters at $1 \mathrm{AU}$, for the time around solar activity minimum, compiled by Schwenn (1990).

\begin{tabular}{lll}
\hline & Low speed wind (LSM) & Fast wind (HSS) \\
\hline Flow speed $v_{\mathrm{p}}$ & $250-400 \mathrm{~km} \mathrm{~s}^{-1}$ & $400-800 \mathrm{~km} \mathrm{~s}^{-1}$ \\
Proton density $n_{\mathrm{p}}$ & $10.7 \mathrm{~cm}^{-3}$ & $3.0 \mathrm{~cm}^{-3}$ \\
Proton flux density $n_{\mathrm{p}} v_{\mathrm{p}}$ & $3.7 \times 10^{8} \mathrm{~cm}^{-2} \mathrm{~s}^{-1}$ & $2.0 \times 10^{8} \mathrm{~cm}^{-2} \mathrm{~s}^{-1}$ \\
Proton temperature $T_{\mathrm{p}}$ & $3.4 \times 10^{4} \mathrm{~K}$ & $2.3 \times 10^{5} \mathrm{~K}$ \\
Electron temperature $T_{\mathrm{e}}$ & $1.3 \times 10^{5} \mathrm{~K}$ & $1 \times 10^{5} \mathrm{~K}$ \\
Momentum flux density & $2.12 \times 10^{8}$ dyne cm $^{-2}$ & $2.26 \times 10^{8}$ dyne cm \\
Total energy flux density & $1.55 \mathrm{erg} \mathrm{cm}^{-2} \mathrm{~s}^{-1}$ & $1.43 \mathrm{erg} \mathrm{cm}^{-2} \mathrm{~s}^{-1}$ \\
Helium content $n_{\mathrm{p}} / n_{\mathrm{He}}$ & $2.5 \%$, variable & $3.6 \%$, stationary \\
\hline
\end{tabular}

The total energy flux density which is also invariant is the sum of two main components: the kinetic energy flux and the potential energy flux (basically the work done for moving the solar wind out of the Sun's gravitational potential). Although these components differ strongly for the two states, their sum is about the same. There is no explanation yet for these strange invariances. We suspect that a crucial key to understanding the solar wind phenomenon is hidden here.

In addition to these two basic states, the slow solar wind filling most of the heliosphere during high solar activity can be considered a third category. It emerges above active regions distributed over large parts of the Sun, far from the heliospheric current sheet, and in a highly turbulent state. It differs in some aspects from the minimum type of slow solar wind. Finally, we regard the plasma expelled from the Sun during huge coronal mass ejections as a category on its own, because of some fundamental differences to be described later.

\subsection{Solar wind in three dimensions}

On the basis of the discoveries in the 1970s, a three-dimensional model of the heliosphere and the stream-structured solar wind had emerged. It is most adequately visualized in terms of the ballerina model first proposed by Alfvén (1977). Figure 8 is an artist's view of the inner heliosphere as it may appear immediately before a typical solar activity minimum, e.g., in 1975. We find the Sun's poles to be covered by large coronal holes. They are areas of open magnetic field lines, the northern hole being of positive (outward directed) polarity, the southern hole being negative. Some tongue-like extensions of the coronal holes reach well into the equatorial regions and give the Sun the appearance of a tilted magnetic dipole. The Sun's equatorial region is governed by bright active centers (including some sunspots left over from the past cycle) and their loop-like and mainly closed magnetic structures above.

What looks like the skirt of a spinning ballerina is the warped separatrix between positive and negative solar magnetic field lines dragged out into interplanetary space by the radially out-flowing solar wind plasma. This separatrix carries an electric current in order to allow the magnetic polarity switch and is thus called heliospheric current sheet. It is formed on top of the closed magnetic structures at the transition between closed and open flux tubes, i.e., generally in the middle of the near-equatorial belt of activity. If the spinning skirt passes an observer sitting, say, at the Earth, he would notice a polarity switch and call it a crossing of a magnetic sector boundary. The size and number of magnetic sectors is closely related to the structure of the underlying corona. i.e., the shape of the activity belt and the coronal holes, respectively. The field lines are curved like Archimedean spirals: they denote the locations of radially flowing plasma parcels that have been released from the same source on the rotating Sun at different times (in honor of their discoverer, these spirals are called Parker spirals, see Hundhausen, 1972). Since the spiral angle $\left(\sim 45^{\circ}\right.$ at 


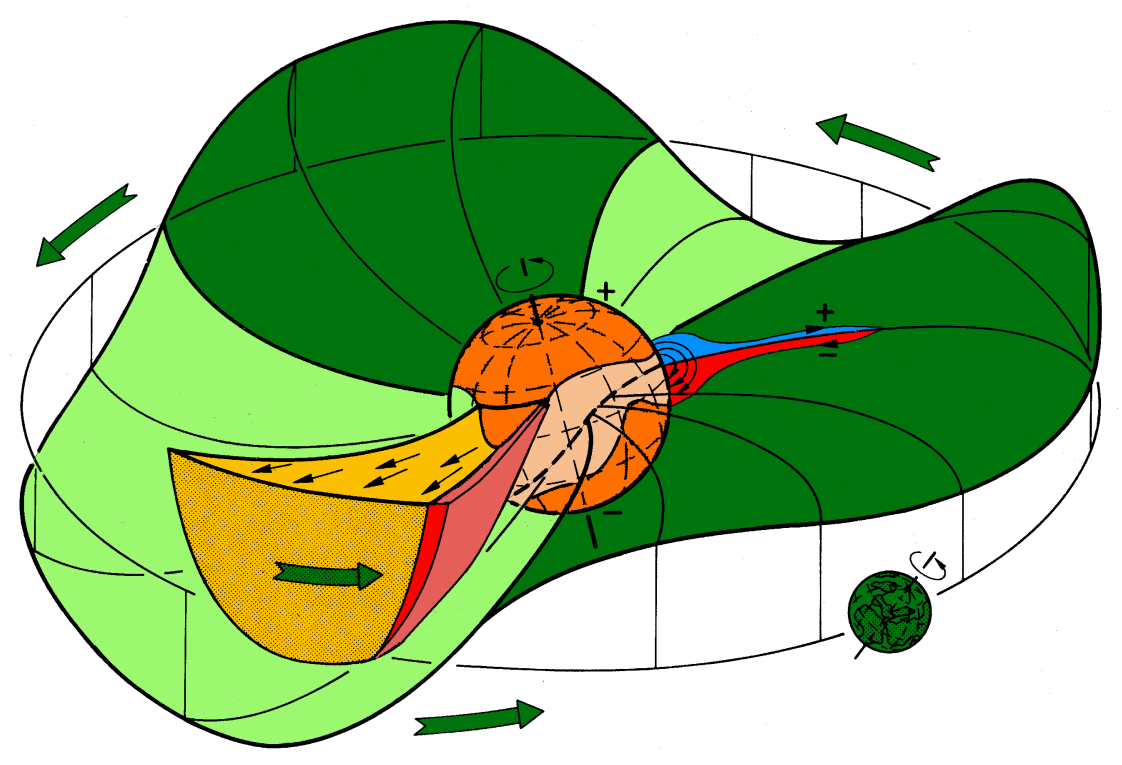

Figure 8: The "ballerina model" of the 3-D heliosphere, according to Alfvén (1977).

1 AU on average) depends on the flow speed, streams of different speed from contiguous sources begin interacting with each other on their way out into the heliosphere. Note though that the spiral winding does not concern the meridional component of the IMF.

The large polar coronal holes are the sources of high-speed solar wind. The emission of slow solar wind is sharply confined to a belt of about $30^{\circ}$ width in latitude centered at the warped current sheet. The warps of both the current sheet (which can be taken to be the heliomagnetic equator, Schulz, 1973) and the coronal hole boundaries with respect to the heliographic equator allow some high-speed streams to extend to low latitudes so that they become observable at times even in the plane of the ecliptic. This occurs preferentially in the 2 years before activity minimum, when the large-scale coronal structure is rather stable, and high-speed streams reappear at the same heliographic longitudes for many solar rotations.

At times of minimum solar activity there are almost no warps left in the current sheet which is then plane like a disk lying very close to the plane of the heliographic equator. This is demonstrated in Figure 9 (from Schwenn et al., 1997) where two images registered by the LASCO coronagraphs on board SOHO in early 1996 were merged. They give a very typical example of the appearance of the extended corona at activity minimum. The inner part was taken in the light of the green coronal emission line at $530.3 \mathrm{~nm}$ produced by Fe XIV ions at temperatures of $2 \times 10^{6} \mathrm{~K}$. This spectral line is particularly well-suited to outline hot magnetic structures in the inner corona. The outer part taken in white light shows the larger-scale electron density distribution.

The large-scale warps of the heliospheric current sheet are caused by localized quadrupole terms of the solar magnetic field. These give rise to similar warps in the coronal hole and stream boundaries and allow them to reach at times across the heliographic equator. Thus, the stream boundaries become effective with respect to longitude. Because of the speed difference, the flows on either side begin interacting with each other with increasing distance from the Sun. In case of

Living Reviews in Solar Physics

http://www. livingreviews . org/lrsp-2006-2 


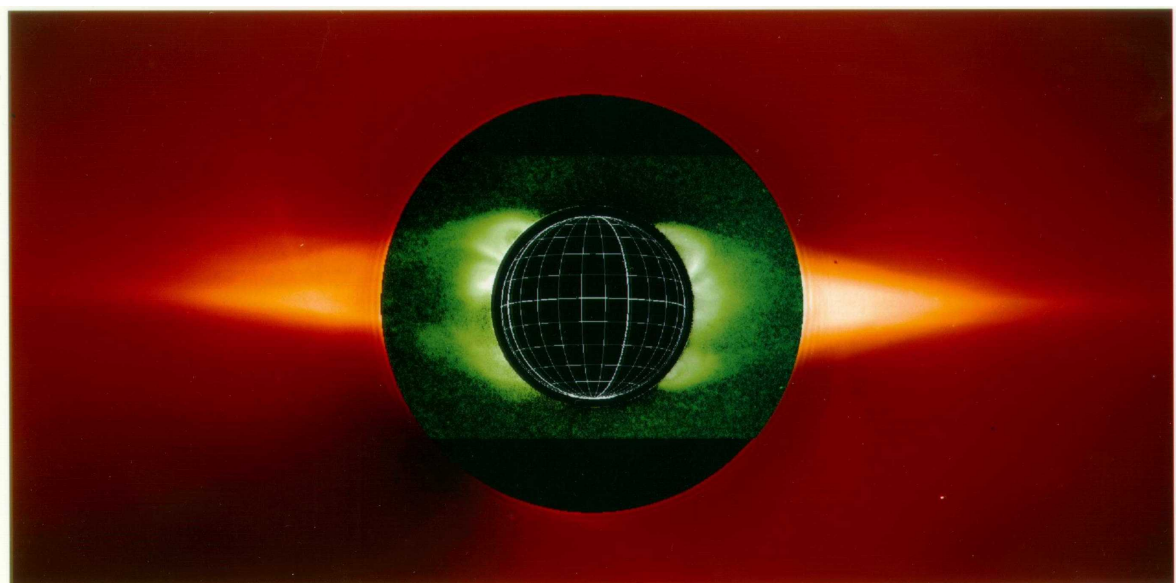

Figure 9: A coronagraph view of the extended minimum corona on February 1, 1996. It is composed from an image taken by the LASCO $\mathrm{C} 1$ coronagraph onboard SOHO in the light of the green coronal emission line at $530.3 \mathrm{~nm}$ (inner part) and a white-light image taken by the LASCO C2 coronagraph (outer part). From Schwenn et al. (1997).

stable conditions, these structures appear to corotate with the Sun and are thus called corotating interaction regions (CIRs).

Figure 10 (from Schwenn, 1990) shows an idealized view of such a CIR and its evolution out to a distance of approximately 1 AU. At the Sun, the stream interface was found to be very abrupt, which is indicated in Figure 11 by the rectangular speed increase. With increasing distance, the faster plasma stream lines, having a smaller Parker angle, start pressing the slow plasma with the more strongly curved field lines. Compression and deflection of the plasma on both sides of the interface finally yield the typical profiles found at $1 \mathrm{AU}$. The total range filled with plasma affected by these interactions amounts to roughly $30^{\circ}$ at $1 \mathrm{AU}$. However, the plasma packed into that range stems from coronal source regions originally spanning some $70^{\circ}$ in longitude. Thus, magnetic sector boundaries are often found close to the stream interfaces at $1 \mathrm{AU}$ and well within the compression regions. Note though that they usually start off at the Sun with considerable separation that often exceeds $30^{\circ}$ (Schwenn, 1990). As a matter of fact, sector boundaries and stream interfaces have basically nothing to do with each other. Their often-observed proximity at 1 AU should be regarded as fortuitous.

Compression and deflection of the solar wind flow in CIRs has an important consequence for the magnetic field: it undergoes the same compression and participates in the deflection process. Thus, there may arise enhanced out-of-ecliptic field components, particularly in the vicinity of magnetic sector boundaries. Here we have identified one mechanism for generating a southward pointing IMF, which is known to drive geomagnetic disturbances at Earth.

At the backside of fast streams, no interaction of the flows occurs and no CIRs develop, since here the different Parker angles lead to a separation of the flows rather than compression. The transition from the fast to the slow state is found to extend over some $60^{\circ}$ at $1 \mathrm{AU}$. However, the location of the original stream boundary can be identified from the abrupt change in the element abundance and the ionization state (Geiss et al., 1995). Further, mapping back the flow to the Sun assuming a strictly radial flow at the locally measured speed, the original rectangular profile at the Sun is nicely reconstructed.

With increasing distance from the Sun, the compression waves at the CIRs steepen to finally form corotating shock waves (see Gosling et al., 1976). There are fast forward shocks at the front side (propagating into the slow-wind side) and fast reverse shocks traveling seemingly backward 


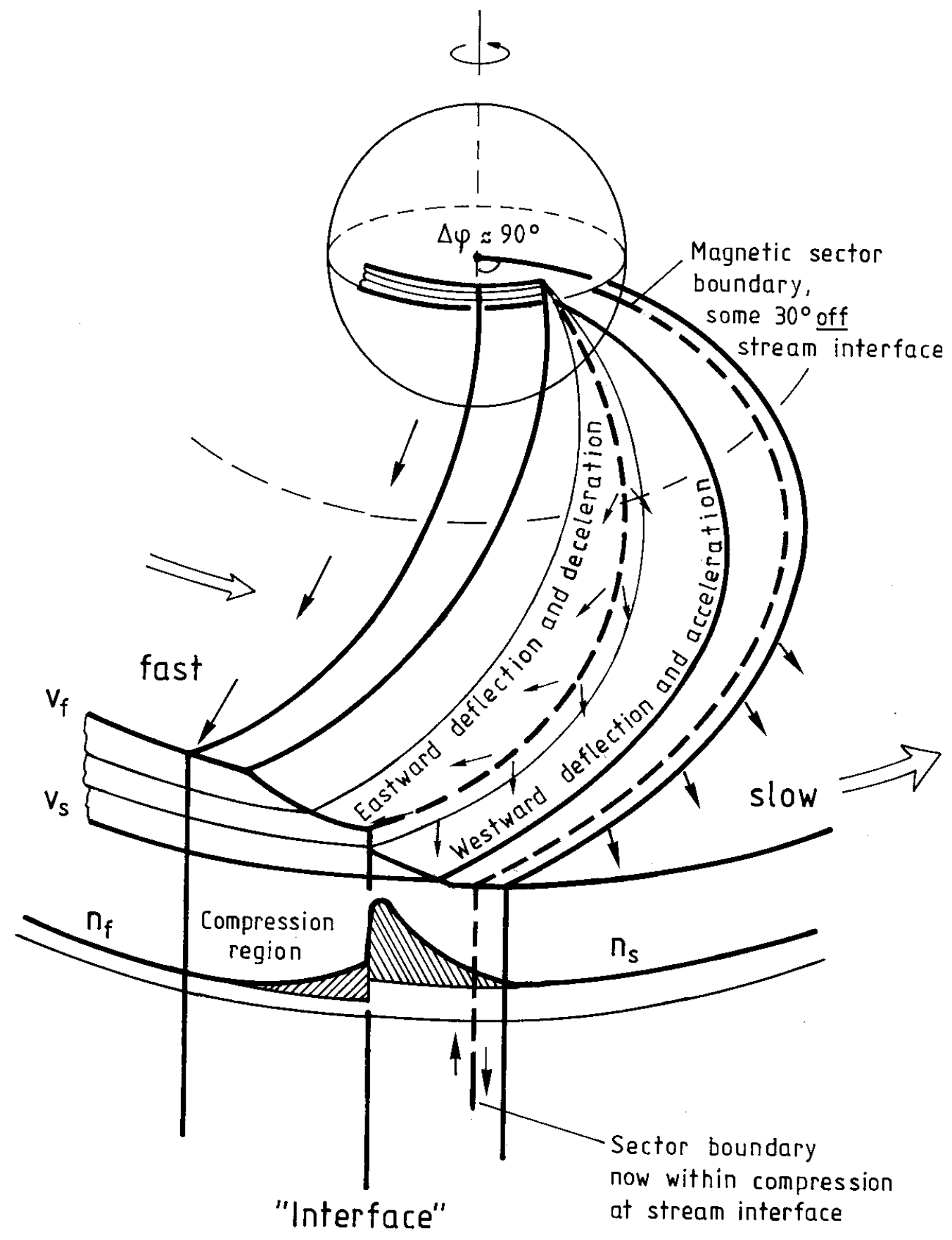

Figure 10: An idealized view of a corotating interaction region (CIR) and its evolution from a rectangular speed profile at the Sun into a more gradual speed increase at 1 AU. From Schwenn (1990). 
(propagating into the fast wind coming from behind). The formation of CIR shocks contributes to further eroding the originally steep stream profiles.

Corotating shocks at CIRs, in a manner similar to that of transient-related interplanetary shock waves and planetary bow shocks, can accelerate ionized particles to considerable non-thermal energies (see, e.g. Scholer et al., 1999). At times near activity minimum CIRs could be identified in plasma measurements by Ulysses (at $4 \mathrm{AU}$ ) at latitudes up to some $40^{\circ}$. At higher latitude, there was nothing but high-speed wind encountered. However, energetic particles apparently associated with CIR shocks have been observed at much higher latitudes (McKibben et al., 1995), to which the CIR shocks do not propagate (Gosling and Pizzo, 1999). That means that there must be a magnetic connection between these high latitudes and those lower latitudes where particles energized at CIRs can be injected. It may be the combined effect of differential rotation of the photosphere, rigid rotation of the corona at the equatorial rate, and the offset between the Sun's rotational and magnetic axes that allows near-equatorial field lines to connect to high solar magnetic latitude regions (Fisk, 1996, see also Posner et al., 2001). This mechanism transfers information on the corotating stream structure to latitudes where the stream structure itself is not discernible.

A second such mechanism results from meridional "squeezing" of compression regions, as had been observed by Burlaga (1983). He concluded that compression regions may extend over larger latitudinal ranges than the high-speed streams causing them. Of course, such squeezing would imply meridional flows (Siscoe and Finley, 1969) and magnetic field excursions within these regions. This is a second mechanism for generating $B_{\mathrm{z}}$ south of the IMF. Without knowing this effect, an observer of geomagnetism at the Earth might be surprised by the sudden appearance of southward $B_{\mathrm{z}}$ completely "out of the blue."

\subsection{Solar wind and space weather}

In the previous Section 2.2, I have already explained why the IMF that usually does not have major meridional components, may undergo substantial deflections leading to geoeffective $B_{\mathrm{z}}$ components that allow magnetic reconnection between the IMF and the Earth's intrinsic field.

The action of potential reconnection is further enhanced by the pressure pulse from the compressed plasma. Rosenberg and Coleman Jr (1980) studied the behavior of $B_{\mathrm{z}}$ around sector boundaries extensively and explained it in terms of the ballerina model (see Figure 8): At a sector boundary the current sheet must necessarily be inclined versus the ecliptic plane, implying the existence of non-zero $B_{\mathrm{z}}$ components. At the transitions from well within one sector (with $B_{\mathrm{z}}=0$ ) to well within the other sector (again with $B_{\mathrm{z}}=0$ ) the observer will see a $B_{\mathrm{z}}<0$ first and, after crossing the stream interface, $B_{\mathrm{z}}>0$. This applies for any sector crossing, positive to negative and vice versa, as long as the general dipole of the Sun maintains its orientation. Once the Sun's dipole is reverted (around solar activity maximum), the sequence of $B_{\mathrm{z}}$ excursions is also reverted. This reversal was indeed confirmed (Rosenberg and Coleman Jr, 1980). The CIR scheme in Figure 10 illustrates that the density profile in the compression region (with its increased ram pressure) is very asymmetric with respect to the sector boundary. Thus it matters a lot on which side the $B_{\mathrm{z}}<0$ excursion occurs, be it on the low pressure side before the boundary or at the high pressure side behind it. This phase shift between $B_{\mathrm{z}}<0$ and the pressure pulse varies with the 22-year magnetic solar cycle and is superimposed on the well-known 11-year modulations. Indeed, there were some unexplained 22-year periodicities in geomagnetism reported by, e.g., Chernosky (1966) and Russell (1974).

There is another fundamentally different mechanisms causing geoeffective $B_{\mathrm{z}}$ south swings:

Solar wind high-speed streams are dominated by large-amplitude transverse Alfvénic fluctuations causing major excursions of both the proton flow and the IMF vector on time scales of minutes to hours (Belcher and Davis Jr, 1971), see also Marsch (1991) and Tu and Marsch (1995). They corotate with the Sun, often for several months. Once these high-speed streams reach the 
Earth, the occasional southward deflections of the IMF due to the Alfvén turbulence stir medium level geomagnetic activity (see Tsurutani and Gonzalez, 1987). Bartels (1932), had postulated "M-regions" on the Sun as sources of these geomagnetic effects. The close association between high-speed streams and M-regions had already been noted in the earliest solar wind observations from the Mariner 2 space probe in 1962 (Snyder et al., 1963, see also Schwenn, 1981). Tsurutani and Gonzalez (1987) and Tsurutani et al. (2004a) inspected the effects of high-speed streams on geomagnetism in terms of what they called "high-intensity long-duration continuous AE activity (HILDCAA) events" (Figure 11). Remember that the compression and deflection of the plasma flow in the CIRs in front of high-speed streams may also lead to geomagnetic activity (Schwenn, 1981). It does not matter whether the steepening at the CIRs has already led to the formation of corotating shocks or shock pairs at the CIRs, a process which only rarely occurs inside the Earth's orbit (see Schwenn, 1990).

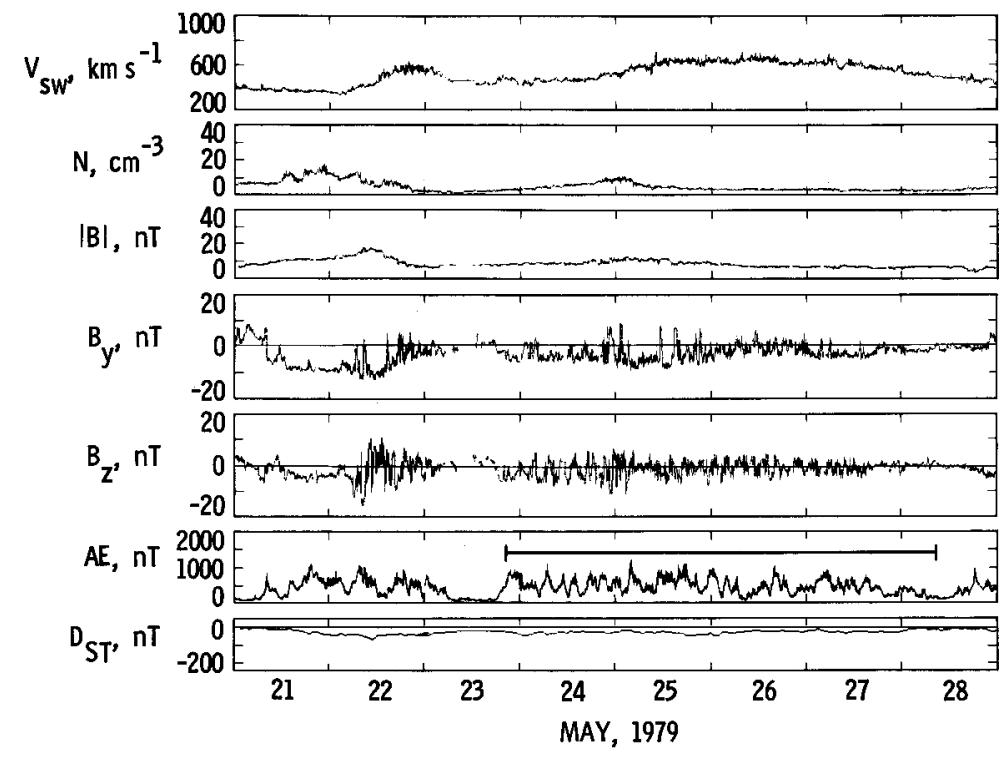

Figure 11: The "high-intensity long-duration continuous AE activity (HILDCAA) event" of May 23 to 28, 1979, and the related Alfvén wave train within the high-speed solar wind stream. The excursions of the geomagnetic AE-index follow the $B_{\mathrm{z}}$ excursions in very much detail, with a time delay of about 100 minutes. Note that within the compression region in front of the stream the $B_{\mathrm{z}}$ component is also negative, and AE reacts similarly. The global $D_{\text {st }}$ index is not affected. From Tsurutani and Gonzalez (1987).

The recurrence of this particular type of geomagnetic activity every 27 days, i.e., exactly in the rhythm of solar rotation, had led Bartels (1932) to postulate the existence of $M$-regions on the Sun already in the 1930s. He thought they were long-lived stable regions on the Sun which emit certain particles capable of stirring geomagnetism. After all, he was strikingly right except for one aspect: these M-regions are not to be sought in active regions on the Sun, as he thought, but rather in the inactive parts: the M-regions are associated with the coronal holes representative of the inactive Sun, and the geomagnetism is stirred by the streams of high-speed plasma (with their Alfvénic fluctuations) emanating from them.

A pretty illustration of the close relation between interplanetary magnetic field, coronal holes, solar wind streams and geomagnetic effects was given by Sheeley Jr and Harvey (1981), shown in Figure 12. In the upper half, all patterns are rather regular products of the inactive Sun. Those 
data were from the three years before activity minimum in 1976. With the new activity rising from 1977 on, transient processes disturbed the regular stream pattern and caused sporadic strong geomagnetic storms: products of the active Sun. It is important in the context of space weather to always remember this distinction. 


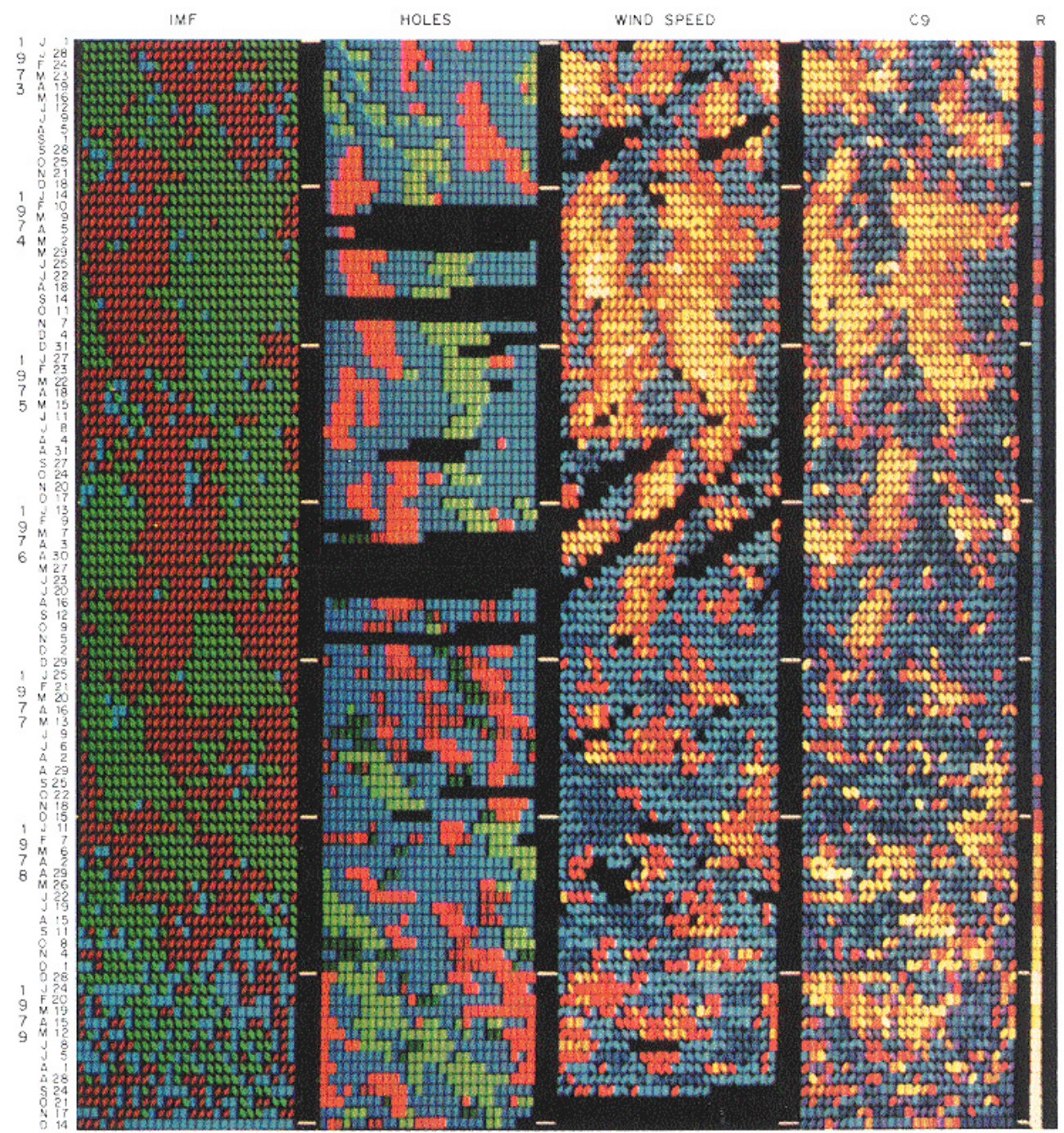

Figure 12: A 27-day Bartels display of IMF polarity, coronal hole occurrence (plus 3 days to allow for Sun-Earth transit time), solar wind speed at Earth, and geomagnetic disturbance index C9. A 27day average sunspot number is indicated in the narrow strip on the extreme right. Coronal holes were counted only when they occurred within 400 of the solar equator. The color coding was chosen such that commonalities are best visible (for details see Sheeley Jr and Harvey, 1981). 


\section{Radiation from Solar Flares}

Solar flares are certainly among the most dramatic and energetic fast processes in our solar system that we know of. The flashes of electromagnetic radiation released within seconds to minutes may cover a wavelength range of as much as 17 orders of magnitude: from kilometric radio waves through the infrared, visible and UV ranges down to X-rays and even Gamma-rays.

\subsection{Some historical remarks}

Major optical flares occur only rarely, depending on the phase of the activity cycle, and they last only a few minutes. So it was by pure luck that Mr. R.C. Carrington on September 1, 1859 at 11:18 GMT, while doing routine sunspot observations, could witness such a unique event for the first time (see the worthwhile original report by Carrington, 1860, reprinted in Meadows, 1970). The brilliancy of the flash equaled that of direct Sunlight, and at first he attributed it to a failure of his telescope. Within the 60 seconds it took him to call a witness, the flash had already much changed and enfeebled. Fortunately enough, Mr. R. Hodgson, another observer at a different location, had also seen the flash and confirmed its existence (Hodgson, 1860, also reprinted in Meadows, 1970). Carrington, in his report to the Royal Society, mentioned the potential connection of this strange solar event with the strong geomagnetic storm that occurred only 17 hours and 40 minutes later. He was honest and careful enough not to overvalue this connection ("One swallow does not make a summer."). However, this discovery can be considered not only as a landmark in modern solar astronomy but also the beginning of space weather research.

This remarkable event was recently revisited by Tsurutani et al. (2005). They used newly reduced and calibrated ground-based magnetometer data and derived characteristic data that can be compared with those from other events in the past 145 years. It turned out that the September 1, 1859 event was among the fastest and most energetic flares ever since (Cliver and Svalgaard, 2004). Of course, there is not much information recoverable about its radiation effects, besides the visual data given by Carrington and Hodgson. But the geomagnetic effects must have been extreme. For example, aurora were sighted to geomagnetic latitudes as low as $20^{\circ}$ (Honolulu), and fires were set by arcings from ground-induced currents (GICs) in telegraph wires, both in Europe and the U.S. (see, e.g., Tsurutani et al., 2003, 2005, and references therein).

For quite some time after the discovery of this most spectacular type of event on the Sun, various expressions have been used to name it. The solar flare nomenclature was investigated by Cliver (1995). He revealed that the term flare can be found for the first time in Bartels (1932)'s famous M-region paper. The unofficial use of this new term can be traced to a paper by Richardson (1944) who stated: "The strongest reason for the adoption of 'flare' is that in one word are combined the most outstanding features of the phenomenon: its sudden appearance, great brilliancy, and rapid variations in intensity."

The flare phenomenon has always attracted the solar physics community. Thousands of flares have been observed in all detail and described in hundreds of articles and books (the interested reader is referred, e.g., to the books "Solar Flares" by Švestka, 1976 and "High Energy Solar Physics" by Ramaty et al., 1996, and to the article by Švestka, 1981, with some 500 references on flare observations, and further to the article in Living Reviews in Solar Physics "Flare Observations" by Benz, 2008). Because of their significance for solar-terrestrial relations (the term space weather has been coined not before the 1980s), flares were regularly observed by "flare patrols" at several observatories around the globe, and agencies like NOAA listed them in their Solar Geophysical Reports for many years. With the advent of regular X-ray observations from space with the SOLRAD satellite in 1968 and the GOES satellites in 1975 a new and more objective classification of flares in terms of X-ray brightness was established (for historical records see http://www.ngdc.noaa.gov/stp/SOLAR/ftpsolarflares.html\#cf). 
Theoreticians and modelers have been busy in finding consistent explanations of the flare phenomenon (see, e.g., the textbook Physics of the Solar Corona by Aschwanden (2004) or the upcoming article "Physics of Solar Flares" in Living Reviews in Solar Physics). However, without going into detail here I dare to state: there is no unique, consistent, and generally accepted explanation yet.

The total energy released in the course of flares can differ by several orders of magnitude: from some $10^{19} \mathrm{~J}$ for the smallest events that are barely recognizable as "events" up to some $10^{25} \mathrm{~J}$ for the most energetic ones. Significant fractions of that energy go into radiation, the rest goes into heating and acceleration of particles, the partition depending on the type of flare.

The different kinds of radiation come from different parts of the flare site and are released at different times of the flare process. In the following sections, I will describe the various emissions organized in a timely order as they appear rather than by their spectral properties.

\subsection{Soft X-rays from flares}

Quite often, the first visible signature of a flare appears in soft X-rays with energies up to some tens of $\mathrm{keV}$. It is thermal flare plasma radiation that signalizes the sudden heating of coronal plasma to temperatures of some $10^{7} \mathrm{~K}$. This radiation is due to the bremsstrahlung continuum and to a multitude of lines of heavily stripped ions, e.g., the line complex around $0.186 \mathrm{~nm}$ from helium-like iron ions. The time profiles of soft X-ray bursts are often very similar to those of simultaneous radio microwaves. Since the onset of regular observations from space in 1968, the intensity of soft X-rays has been used for flare classification. It is based on measurements (using calibrated satellite-carried instruments) of the soft X-ray emission in the 0.1 to $0.8 \mathrm{~nm}$ band as published in real-time by NOAA (http://www.sec.noaa.gov/today.html). For example, the big X-ray flare on October 28, 2003 was classified as X 17.2, corresponding to the measured power of $1.72 \mathrm{mWm}^{-2}$ (see Figure 4). NOAA is presently operating its own Soft X-ray Imager (SXI) as part of their space weather service (see http://www.sec.noaa.gov/sxi/index.html). More information on presently ongoing observations of solar soft X-ray emission and flares can be found on the websites for the RHESSI mission (http://hesperia.gsfc.nasa.gov/hessi/), the Hinode mission (http://hinode.nao.ac.jp/index_e.shtml), and not to forget the excellent presentation in Wikipedia (http://en.wikipedia.org/wiki/Solar_X-ray_astronomy).

\subsection{EUV and visible light}

At lower levels of the solar atmosphere, EUV emission lines allow a view on a region called flare transition layer that is also heated up quite abruptly (see example in Figure 1). From the upper chromosphere we receive the strongest signal in the Lyman-alpha line (at $121.6 \mathrm{~nm}$ ) and the other members of the Lyman series of hydrogen. Lower down in the solar atmosphere, line emission from the Balmer series of hydrogen becomes dominant, with its most prominent member, the famous $\mathrm{H}$-alpha line at $656.3 \mathrm{~nm}$. In fact, most flare observations have been made using this line, since it is situated near the peak of the visible part of the solar spectrum and is thus easily accessible to ground-based observers. That is the reason why for many years the area at the time of maximum $\mathrm{H}$-alpha brightness of flares and also their "importance" (faint, normal or brilliant) have been used for flare classification. Many volumes of Solar and Geophysical Data beginning in 1938 (published by NOAA) are filled with these data, and yet these lists are incomplete and suffer from nonobjective judgments of different observers. A typical $\mathrm{H}$-alpha flare observed from the ground is shown in Figure 13.

Living Reviews in Solar Physics

http://www. livingreviews . org/lrsp-2006-2 


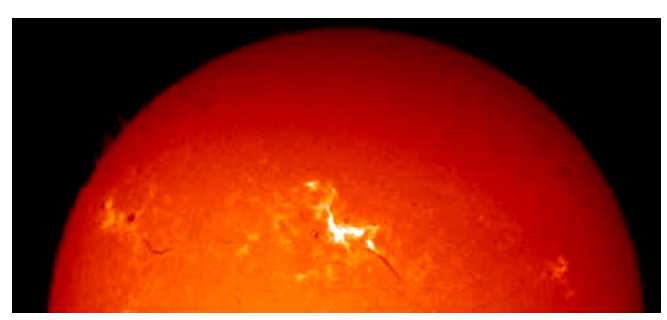

Figure 13: Still from a movie showing The flare on June 7, 2000, 1526-1621 UT, as seen by the H-alpha telescope (HASTA) in El Leoncito, Argentina http://www.oafa.fcefn.unsj-cuim.edu.ar/hasta/. (To watch the movie, please go to the online version of this review article at http://www.livingreviews.org/ lrsp-2006-2.)

\subsection{Hard X-rays}

During the flare flash phase, usually a few minutes after the soft X-ray burst, another kind of apparently non-thermal radiation (as first noted by De Jager, 1965) is observed for many but not all flares: hard X-rays with energies of tens of $\mathrm{keV}$ up to a few $\mathrm{MeV}$ in extreme cases (see, e.g. Garcia, 2004). This radiation is due to electrons accelerated to very high energies right at flare onset which then hit the atoms of the lower denser layers and produce a bremsstrahlung continuum in the form of hard X-rays.

Since 2002, the RHESSI mission (Ramaty and Mandzhavidze, 2000; Lin et al., 2003; Hurford et al., 2003), provides high-resolution imaging and spectroscopy from soft X-rays to Gamma-rays. New results are being published in a fast pace (see the ADS for authors like Lin, Krucker, Hudson, Hurford and others).

\subsection{Impulsive microwave bursts}

Simultaneously, this same electron population, in conjunction with strong chromospheric magnetic fields, produces gyro-synchrotron radiation that can be observed from the ground using radiotelescopes (see, e.g. Pick et al., 1990). There is a very good time correlation between the hard $\mathrm{X}$-ray bursts and impulsive microwave radiation, in particular for the higher frequencies beyond $1 \mathrm{GHz}$. The spectrum is a broadband continuum with peak intensities at some tens of $\mathrm{GHz}$ for the strongest events. For detailed information see, e.g., Benka and Holman (1992) and Holman (2003).

Recently, a new kind of rapid solar spikes (100-500 ms) was observed at submillimetric waves (212 and $405 \mathrm{GHz}$ ) by the new Solar Submm-wave Telescope (SST) by Kaufmann et al. (2003). They suggest that these pulse bursts might be representative of an important early signature of CMEs, but a consistent explanation is still lacking. During the November 4, 2003 flare, a further new microwave burst spectral component was observed by Kaufmann et al. (2004), that apparently peaks in the $\mathrm{THz}$ to infrared range. The origin of these bursts is still unclear. This type of radio bursts might be common to many solar events, but observing them requires new techniques that are able to bridge the gap between electronics and photonics.

\subsection{Type IV radio bursts}

Some of these impulsive high-frequency microwave bursts are accompanied by long-lived radio enhancements at lower frequencies. Such type IV radio bursts cover a continuous spectrum with frequencies below $200 \mathrm{MHz}$, i.e., in the meter wavelength range. Sometimes their sources are seen to move away from the Sun. These moving type IV bursts were thought to be caused by electrons 
trapped in a closed magnetic cloud ejected from the flare site, but this interpretation is still under debate. For more information see, e.g., Švestka (1981) or Kahler (1992).

\subsection{Gamma-rays (and white light)}

Protons are also accelerated during the flare process. They can penetrate deeply into the solar atmosphere, into layers which are usually not involved with the flare. These protons can excite both: white light emission and Gamma-rays. Proton energies exceeding some $20 \mathrm{keV}$ are required. This explains why only the really big flares are visible in white light (remember Carrington's observation in 1859) and Gamma-rays.

There are at least six different physical processes that contribute to Gamma-ray emission: 1) electron bremsstrahlung continuum emission, 2) nuclear de-excitation line emission, 3) neutron capture line emission, 4) positron-electron annihilation line emission at $0.511 \mathrm{MeV}, 5)$ pion-decay radiation at $>50 \mathrm{MeV}$ (pions may be generated whenever protons are accelerated into the $1 \mathrm{GeV}$ energy range), and 6) neutron production. The strongest lines in the Gamma-ray spectrum of flares are the positron-electron annihilation line at $511 \mathrm{keV}$ and the $2.223 \mathrm{MeV}$ line produced when neutrons are captured by protons (see, e.g., Ramaty et al., 2002; Lin et al., 2003 and Figure 14). Note that both the positrons and the neutrons must first be produced in the flare process itself. The hardest Gamma line detected so far is the $6.129 \mathrm{MeV}$ line due to ${ }^{16} \mathrm{O}$ nuclei de-excitation. The electron bremsstrahlung continuum was found with energies up to some GeV (Kanbach et al., 1993). It consists of two types: from electrons directly accelerated in the flare and from secondary electrons and positrons released in high-energy reactions (involving pion decay and muon production). For further details see, e.g., Rieger and Rank (2001).

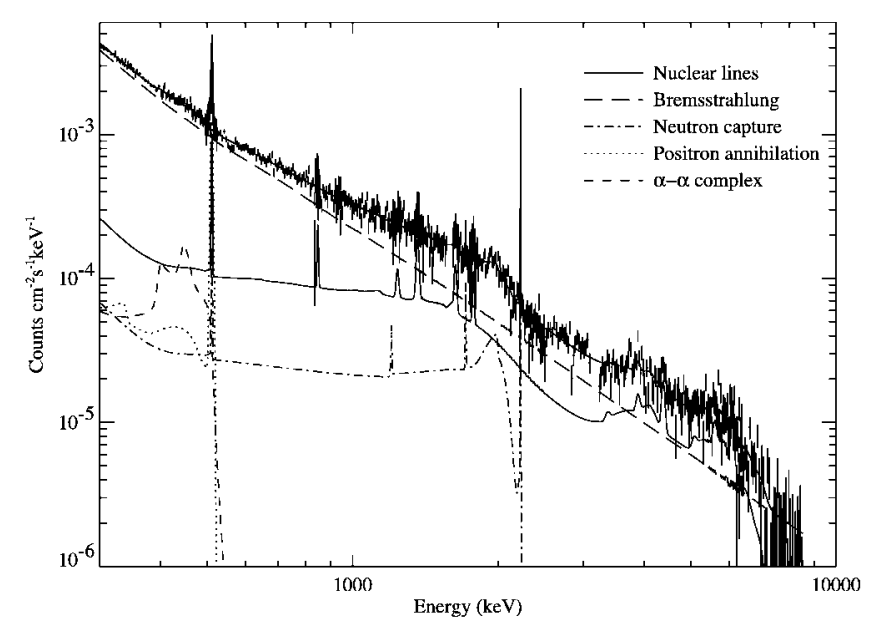

Figure 14: RHESSI Gamma-ray count spectrum from 0.3 to $10 \mathrm{MeV}$, integrated over the interval 0027:200043:20 UT. The lines show the different components of the model used to fit the spectrum. From Lin et al. (2003).

\subsection{Type III radio bursts}

Major fractions of the flare-accelerated electrons and protons escape into space (to be discussed in more detail in the next Section 3.9), guided by the magnetic field lines that are carried out into the heliosphere by the evolving solar wind. Injections of electrons in the keV energy range are

Living Reviews in Solar Physics

http: //www . livingreviews . org/lrsp-2006-2 
accompanied by radio wave emission with frequencies from $\mathrm{MHz}$ down to few $\mathrm{kHz}$. These so-called type III radio bursts (Wild, 1950) are generated in a two-step process (see also Figure 15 from Gurnett et al., 1980): 1. electrons accelerated in solar flares to energies of some keV are streaming away from the Sun and excite plasma oscillations locally, all the way from the corona into the distant heliosphere, the frequency $f_{\mathrm{p}}$ being determined by the local electron density $n_{\mathrm{e}}$ :

$$
f_{\mathrm{p}}=9 \times \sqrt{n_{\mathrm{e}}},
$$

with $f_{\mathrm{p}}$ in $\mathrm{kHz}$ and $n_{\mathrm{e}}$ in $\mathrm{cm}^{-3}$. 2. These plasma oscillations (sometimes also called Langmuir waves) are converted to escaping electromagnetic radiation (of the same frequency or its harmonic) by non-linear wave-wave interactions. The resulting radio waves can be picked up by appropriate receivers in space. Because of the outward travel of the electrons through the radial density gradient the wave frequency gradually decreases with time, and their onset times are gradually delayed. That leads to the characteristic frequency variation of type III bursts as shown in Figure 16 from Kellogg (1980). In many cases, there is an overlaid strong and more spiky signal at very low frequencies observed. It is caused by the plasma oscillations excited locally upon arrival of the electrons at the position of the observing spacecraft. In the case shown in Figure 16, the local plasma density of $7.5 \mathrm{~cm}^{-3}$ would correspond to a local plasma frequency of $24.6 \mathrm{kHz}$, which is indeed at the intensity maximum of the observed plasma oscillation spectrum. Note that the onset times of the oscillations and the waves are almost identical.

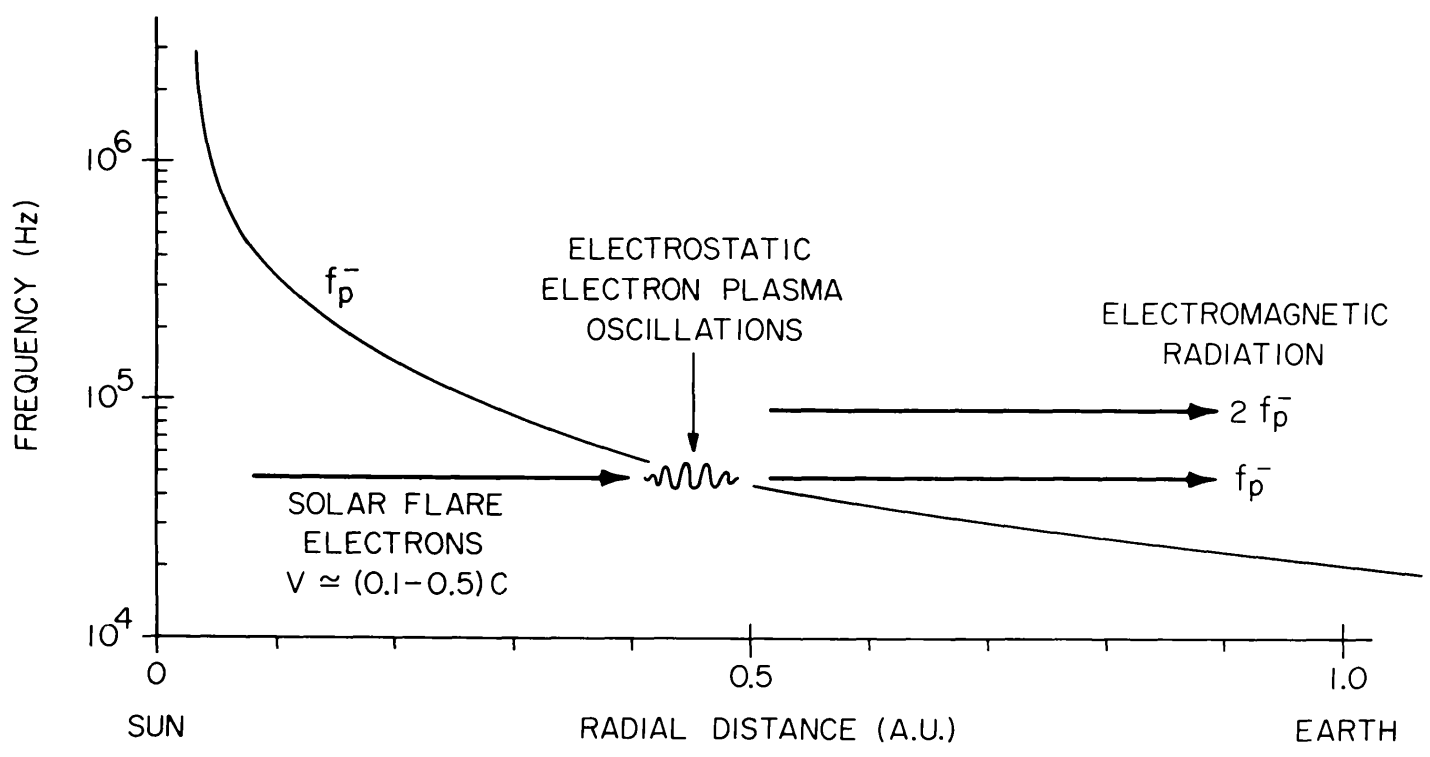

Figure 15: A representative radial profile of the electron plasma frequency in the solar wind illustrating the generation of electron plasma oscillations and the subsequent electromagnetic radiation at the plasmafrequency and its harmonic. From Gurnett et al. (1980).

Modern antenna systems onboard spaceprobes allow determination of the direction of the source of the type III radiation. The frequency itself is a measure of the source's plasma density which, in conjunction with an assumed density model, is a measure of the radial distance of the source. This way, the location of any particular radio source along the path of the type III electrons can be determined. Indeed, the actual shape of the Parker spiral along which these electrons have to 


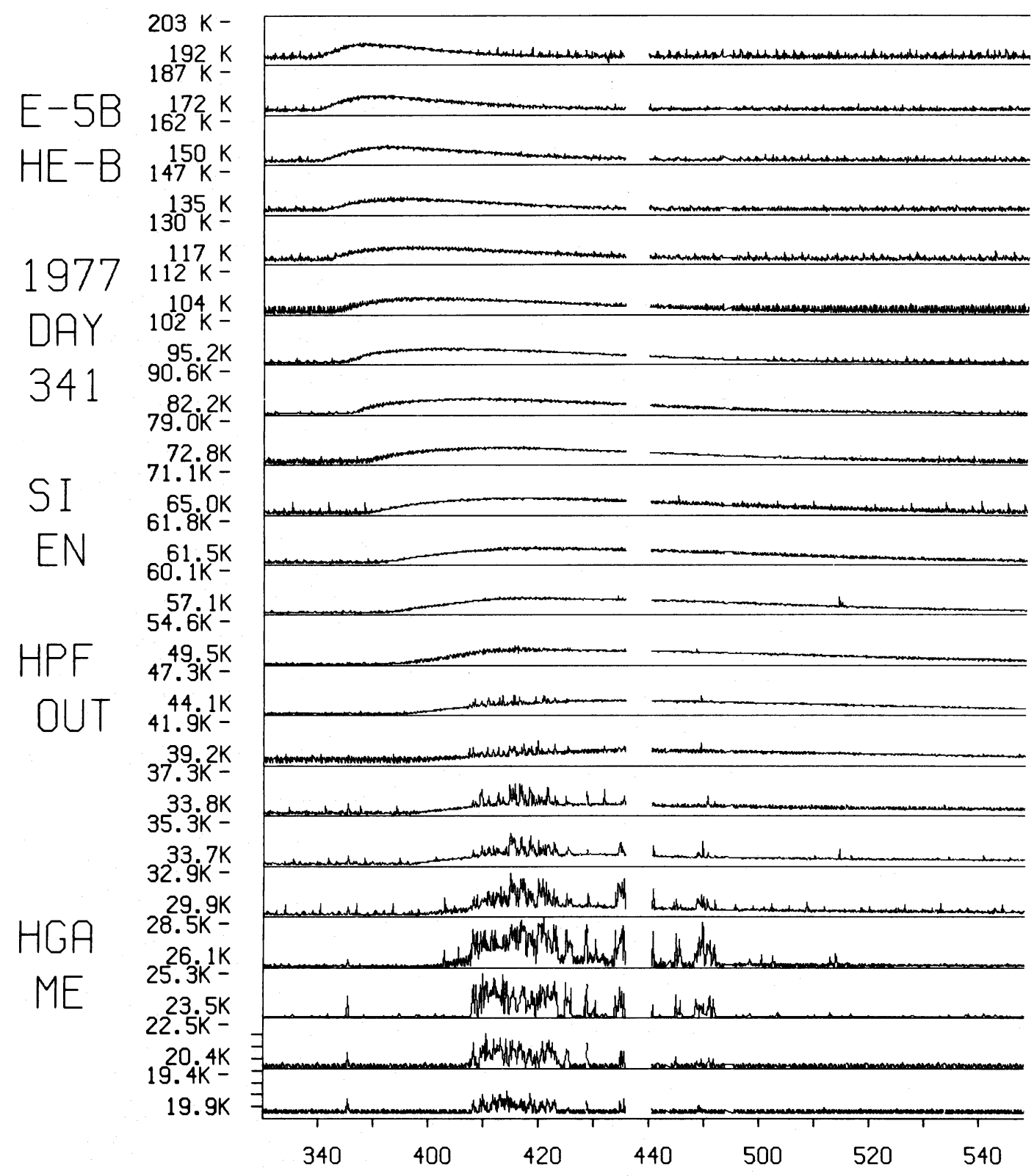

Figure 16: Signal amplitude for a number of radio wave channels during the type III radio burst on December 7, 1977, as observed from Helios 2. From Kellogg (1980). 
move could be experimentally verified (Reiner et al., 1995). Simultaneous radio measurements from two distant spacecraft allow rather precise triangulation of the radiation sources and a stereoscopic view of the electron beam path, even without any assumption of a density profile (Baumback et al., 1976; Gurnett et al., 1978).

The type III electrons have a rather wide energy spectrum. Thus, their travel times to an in situ observer located at some distance from the Sun will vary considerably. That is indeed observed, as is shown in the bottom panel of Figure 17 (from Lin, 2005): the $27 \mathrm{keV}$ electrons arrive about 20 minutes later than the $517 \mathrm{keV}$ electrons.

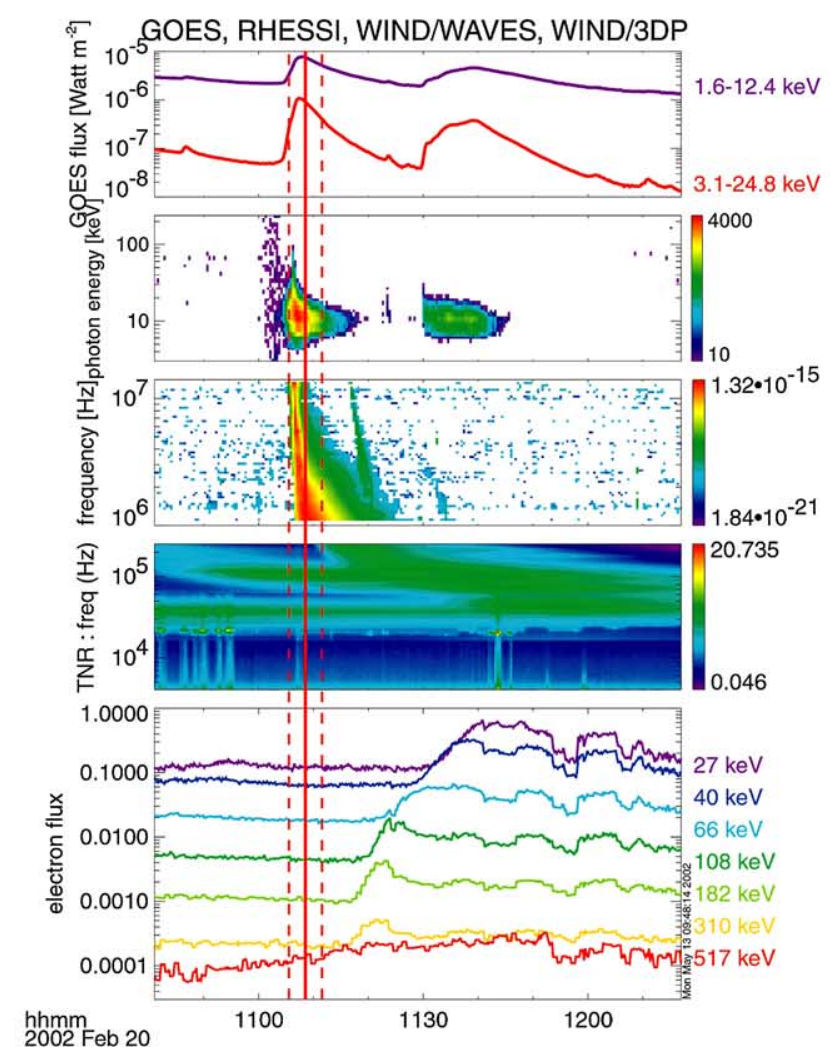

Figure 17: Example of a flare hard X-ray burst observed by RHESSI with corresponding solar type III radio burst and energetic electrons (and Langmuir waves) observed in situ by the WIND spacecraft (Krucker and Lin, 2002). Top panel: GOES soft X-rays; second panel: Spectrogram of RHESSI X-rays from 3 to $250 \mathrm{keV}$; third and fourth panels: radio emission observed by the WIND WAVES instrument; fifth panel: Electrons from $\sim 20$ to $\sim 400 \mathrm{keV}$ observed by WIND 3-DP instrument. From Lin (2005).

\subsection{Metric type II radio bursts}

Many strong flares are accompanied by so-called type II radio bursts (for an early review see Nelson and Melrose, 1985). They appear as stripes of enhanced radio emission slowly drifting from high to low frequencies in dynamic radio spectra. The frequency of this characteristic radiation drops from several hundred $\mathrm{MHz}$ to about $20 \mathrm{MHz}$ or even less within some tens of minutes. The equivalent wavelength of this radiation is in the meter range, and thus these bursts are often 
called metric type II radio bursts. An impressive example from Cane and Erickson (2005) is shown in Figure 18. In this particular case the first harmonic was also observed, as is often the case. It is generally thought that type II radio bursts are due to electrons accelerated at an outward propagating coronal shock front. The decrease in frequency corresponds to motion of the shock through the radial plasma density profile. For a known (or assumed) density profile, one can deduce the propagation speed of the driving shock wave.

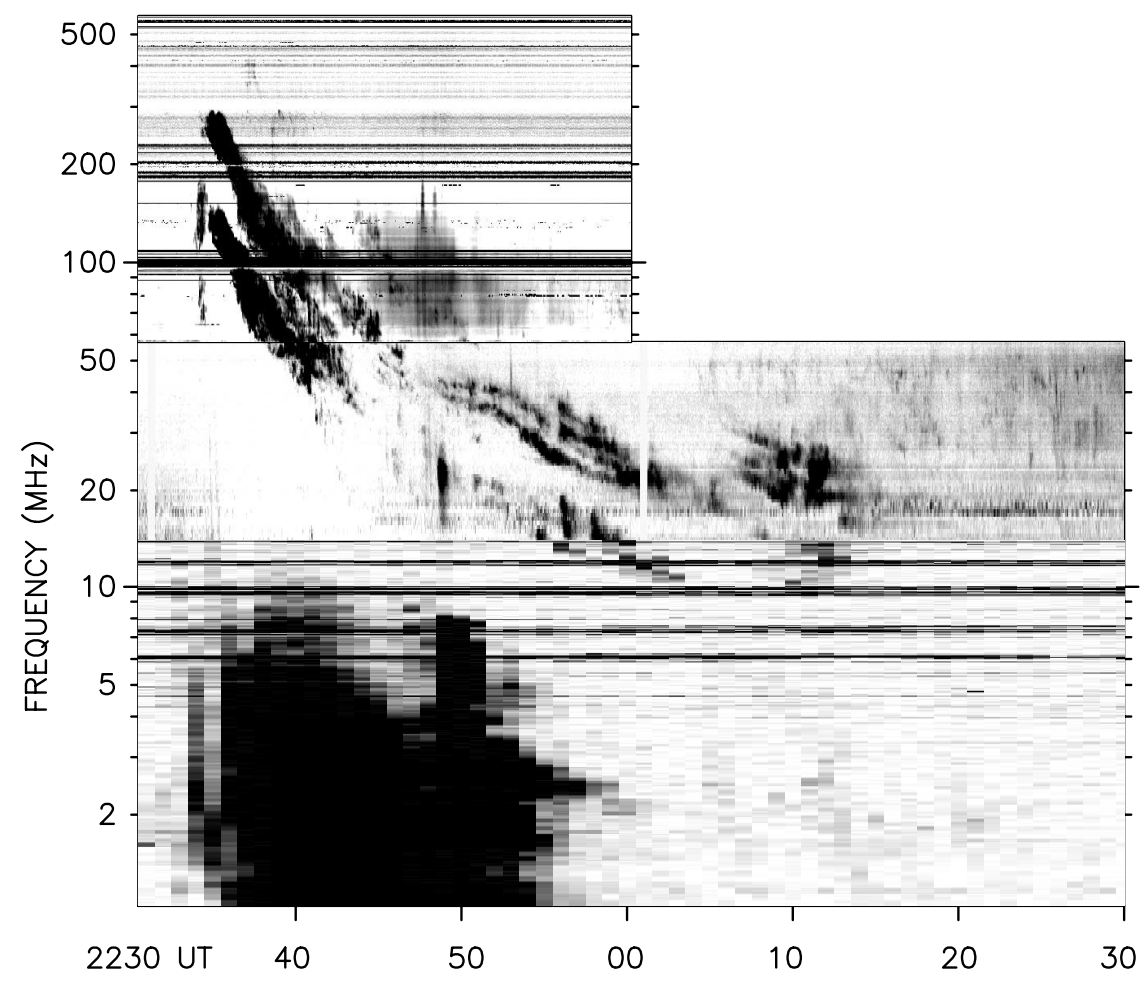

Figure 18: The radio type II burst of November 1, 2003, from 22:00 UT on. This is a clear example of a strong metric type II burst that extends from 300 to $10 \mathrm{MHz}$. It was observed from 3 independent instruments: from the WAVES instrument on the WIND spacecraft and the ground-based helio-spectrogtraphs. The WAVES data cover the frequency range below $14 \mathrm{MHz}$, the BIRS data run from 14 to $57 \mathrm{MHz}$, and the Culgoora data run from 57 to $570 \mathrm{MHz}$. Fundamental and harmonic bands of the type II are easily seen, along with splitting of each band. After the type II bands, some broadband type IV is seen in the Culgoora and BIRS data. A short, type II like feature is seen in the BIRS data between 23:06 and 23:14 UT. The light area in the BIRS data between 22:34 and 22:48 UT results from ionospheric absorption of the galactic background due to solar UV. From Cane and Erickson (2005).

Sometimes the main trace of the radio-signature in the frequency-time plot is accompanied by a second component from apparently much faster propagating features. It appears as rapidly drifting (some $10 \mathrm{MHz} / \mathrm{s}$ ) stripes of enhanced radio emission shooting up from the "backbone" (i.e., the main type II trace in the frequency-time plot) towards both high and low frequencies (see Figure 19 from Mann and Klassen, 2005). These "herringbones," as Roberts (1959) called them, are regarded as signatures of upward and downward pointed electron beams, which are accelerated by the shock waves associated with the "backbone". Mann and Klassen (2002) found some morphological differences between these herringbone electrons and the type III electrons: the latter ones are faster by about a factor of 2 and apparently result from a different acceleration

Living Reviews in Solar Physics

http://www. livingreviews.org/lrsp-2006-2 
process near the flare site.

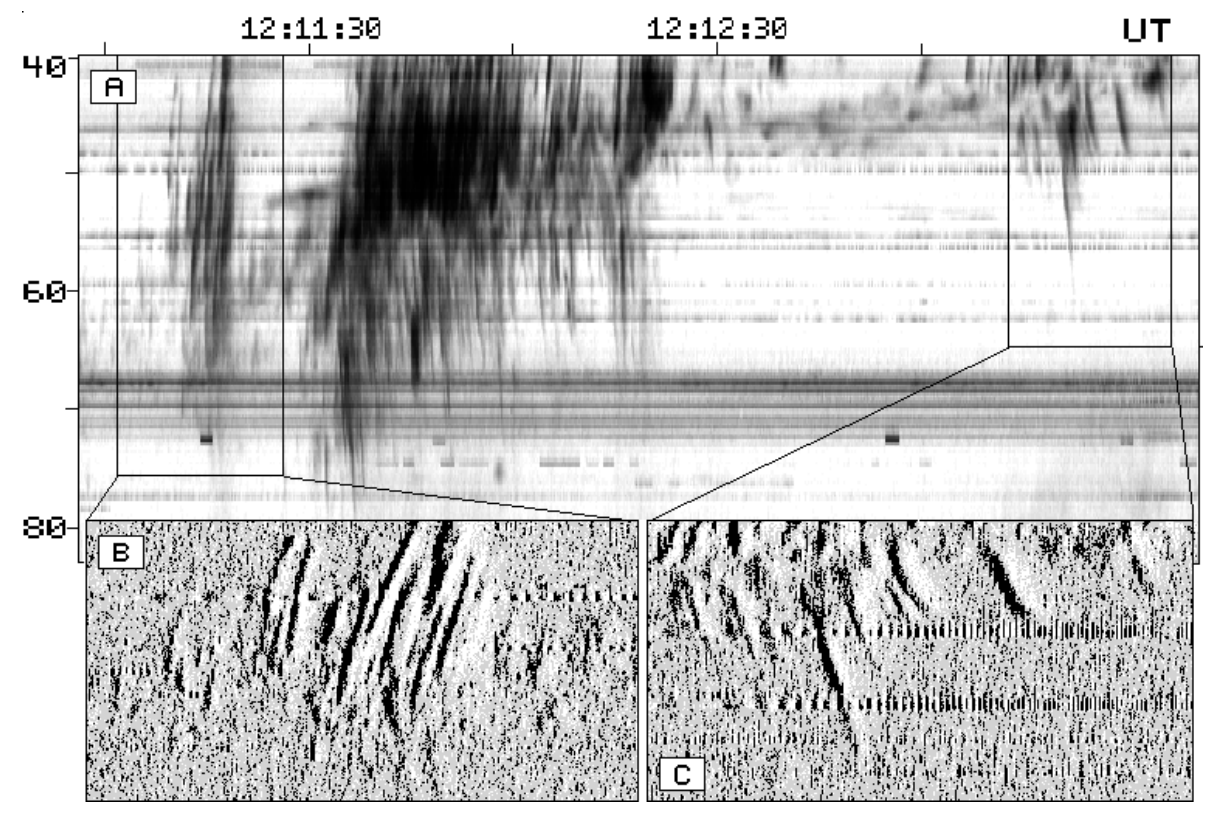

Figure 19: Dynamic radio spectrum (A) of the solar type II burst during the event on June 30, 1995. The "backbone" is slowly drifting from 60 to $42 \mathrm{MHz}$. The "herringbones" are nicely visible during the whole event. The radio intensity is coded by grey scale. The bottom frames (B, C) present the intensity time derivative of the individual "herringbones." From Mann and Klassen (2005).

\subsection{Kilometric type II radio bursts}

There is another type of solar radio bursts, called the kilometric type II radio bursts. They also drift in frequency, from a few $100 \mathrm{kHz}$ to some tens of $\mathrm{kHz}$ over time scales of several days. Cane (1985) had established that the kilometric radio bursts are due to electrons accelerated at interplanetary shock waves on their way from the Sun out into space. In that respect, these kilometric radio burst are similar to the metric radio bursts. In some cases, the shock motion can be well traced from the Sun to the Earth orbit and often far beyond (see, e.g. Reiner et al., 1998, 2001), as is illustrated in Figure 20.

There is still a controversial discussion going on about the nature of the respective shocks causing the radio bursts. Cliver and some colleagues (Cliver et al., 1999, 2004; Cliver, 1999) argue that both the metric and the kilometric burst are produced by CME-driven shocks. The opposing position is held, e.g., by Cane and Reames (1988); Gopalswamy et al. (1998); Cane and Erickson (2005). They argue that the metric radio bursts stem from coronal shock waves driven by flares as blast waves, as suggested before by, e.g., Wagner and MacQueen (1983) or Sheeley Jr et al. (1984). In fact, there is never a continuous spectrum seen connecting the metric and the kilometric radio ranges. This gap had often been assigned to instrumental shortcomings. Only recently, the whole spectral range could be covered, but the gap in the spectrum usually remains. Most metric type II bursts appear to die out within $2 R_{\mathrm{S}}$ (from Sun center) since their frequency rarely drops further than $5 \mathrm{MHz}$. This suggests that they are driven by shock waves that die out soon. On the other hand, a CME driven shock cannot be formed unless the CME speed exceeds the local fast magnetosonic speed. For a majority of the slower CMEs this stringent condition is not fulfilled 


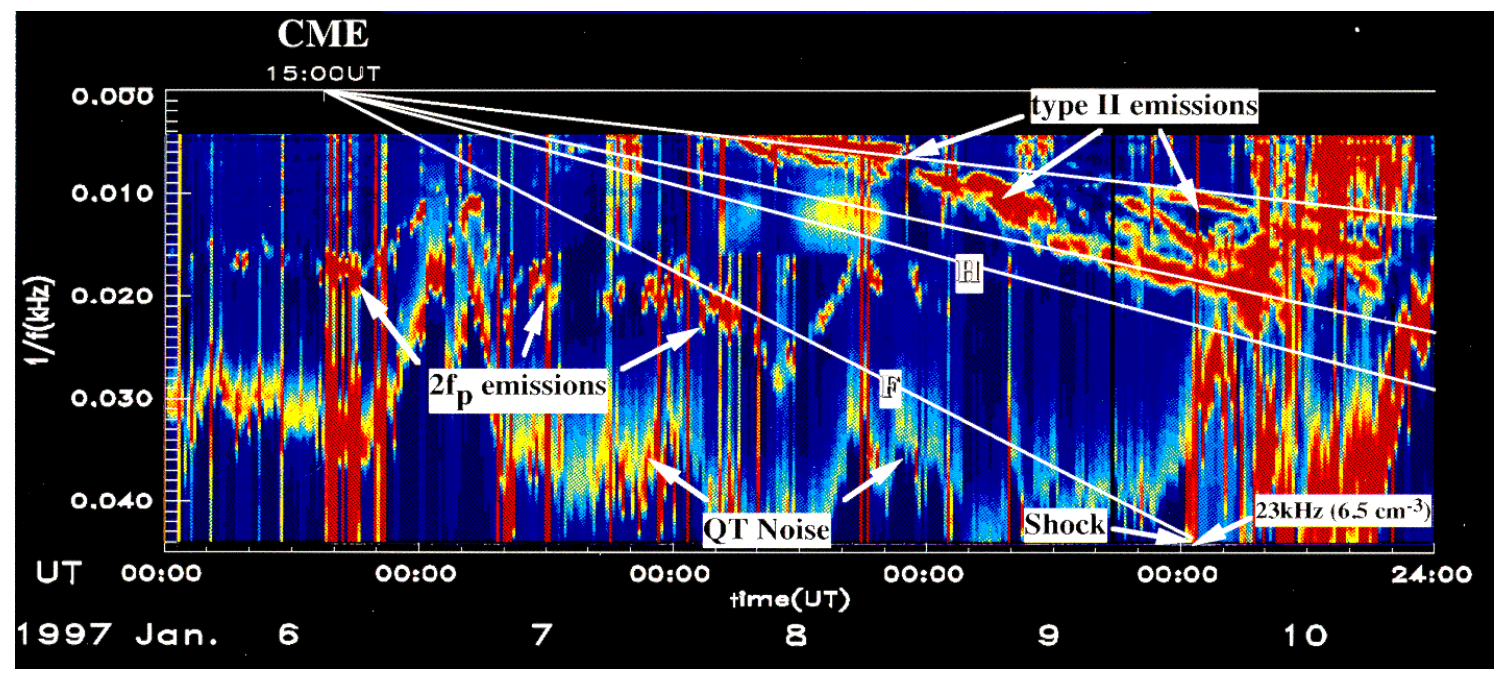

Figure 20: Dynamic spectra of the WIND-WAVES radio data from January 6 to 11, 1997 in the frequency range from 23 to $245 \mathrm{kHz}$. The ordinate scale is the inverse of the observing frequency. The observed type II radio emissions for this event are bracketed by the upper two lines that originate from the CME lift-off time at 15:00 UT on January 6. For a more detailed description see Reiner et al. (1998).

before the CMEs reach a distance of several $R_{\mathrm{s}}$ (Mann et al., 2003). That would explain why the kilometric radio bursts rarely have frequencies above $1 \mathrm{MHz}$. In some cases, both radiation types are seen simultaneously at different frequencies. That does not necessarily prove the simultaneous existence of 2 shocks. There might as well be one large shock front that manages to accelerate electrons at very different distances from the Sun.

The controversy is still open. The interested reader may wish to look up, e.g., Kahler (1992); Gopalswamy et al. (2005a); Cane and Erickson (2005); Reiner et al. (2005) and other articles by these authors and others such as Mann, Klassen, Reames, Kaiser, Webb, to be found using the NASA ADS.

\subsection{Solar flares and space weather}

The various types of radiation produced during the flare process are of interest in the space weather context. The EUV radiation in particular the Lyman-alpha radiation at $121.6 \mathrm{~nm}$ wavelength is absorbed in the Earth's atmosphere and causes its instantaneous heating and expansion. Earthorbiting satellites cruising in low orbits may sense a sudden drag that lowers their orbits (see Figure 5 in Lean, 1991). Any direct damage from the X-ray and Gamma radiation dose to technical systems and humans in space has not been reported, the intensities are probably too low.

The judgment of the role of flares on space weather in terms of geomagnetic effects has changed dramatically in recent years. Since Carrington's discovery of the apparent connection between strong flares and geomagnetic activity in 1859, this connection has been considered a cause and effect relation for many years, despite some obvious shortcomings. Only in the 1980s, it became clear that the only type of solar transient that has a unique cause and effect relation to geomagnetic activity lies in CMEs, not in flares. Schwenn (1983) and Sheeley Jr et al. (1983, 1985) showed that every CME launched with a speed exceeding $400 \mathrm{~km} / \mathrm{s}$ eventually drives a shock wave, which then can be observed in situ, provided that the observer is located within the angular span of that CME. If this shock and the often times following ejecta cloud hits the Earth, geomagnetic effects may occur (provided some conditions on the orientation of the interplanetary magnetic field

Living Reviews in Solar Physics

http: //www. livingreviews.org/lrsp-2006-2 
are also fulfilled). In reverse, every shock wave observed in space (except the ones at corotating interaction regions) can uniquely be associated with an appropriately pointed CME at the Sun. This proves that there is a causal chain linking CMEs to geomagnetic effects. No similar statement can be made for flares. Indeed, there are many CMEs (with geoeffects) without associated flares, and there are flares without associated CMEs (and without geoeffects). The longstanding "flare myth" was finally abolished (see Gosling, 1993; Reames, 1999). However, for the very big and most dangerous events like the one Carrington happened to witness, strong X-ray flares and large CMEs usually occur in a close timely context (Švestka, 2001). It is now commonly thought that both: flares and CMEs, are just the symptoms of a common underlying "magnetic disease" of the Sun (Harrison, 2003).

A very different aspect in space weather issues concerns the radio bursts (not to mention here their direct effects on cell phones and the GPS system, which are addressed in the Living Reviews in Solar Physics article "Space Weather: Terrestrial Perspective" by Pulkkinen, 2007). The type III radio bursts can be tracked through large parts of the heliosphere and help to locate their probable source regions near the Sun. The curvature of the Parker spiral outlined by the type III electrons provides information on the solar wind environment through which theses electrons are passing. Even more important are the metric and kilometric type II radio bursts in that they show the motion of interplanetary shocks from the Sun up to Earth and further out. Thus, this information can be used for practical space weather analysis and forecasting. 


\section{Solar Energetic Particles (SEPs)}

The various highly dynamic processes in the magnetized coronal and interplanetary plasma can cause major acceleration of the charged particle populations. The main locations for electron and ion acceleration are flare sites and shock waves in the corona and in interplanetary space. The energy of these solar energetic particles (SEPs) reaches from a few keV of "suprathermal particles" to some $\mathrm{GeV}$. Sometimes the fastest particles obtain more than half the speed of light, and they arrive at the Earth only a few minutes after the light flash. They are of particular concern in the space weather context since they can penetrate even the skins of spaceprobes traveling outside the Earth's magnetosphere and blind or even damage sensitive technical systems. The strongest events like the ones in August 1972 or in October/November 2003 produce radiation doses that might be lethal to unprotected astronauts while traveling in space outside our protective magnetosphere (see, e.g. Turner, 2001). For the very largest events, SEP ionization of the polar atmosphere produces nitrates that precipitate to become trapped in the Earth's polar ice. Ice core analysis revealed that the largest SEP event in the last 400 years appears to be related to the giant flare observed by Carrington in 1859 (Reames, 2004). Forecasting such extraordinary events is still not possible, for two main reasons: 1) We have not yet identified unique signatures for the driving flare that would indicate an imminent explosion and its probable onset time, location, and strength, and 2) the size of the SEP fluxes is highly variable and appears to be only loosely related to the strength of the flare.

For illustration we show what happened during the dramatic Halloween events in late 2003. In Figure 21 (from Mewaldt et al., 2005) the SEP intensities for electrons and protons as measured by the GOES/SAMPEX/ACE satellites in several energy bands are plotted. The largest of the five major SEP events reached its peak intensity during shock passage at Earth on October 29. It had been launched in context with the X 17.2 flare and the associated halo CME about a day earlier. The maximum energy of probably more than $1 \mathrm{GeV}$ was sufficient for the particles to penetrate the whole SOHO spacecraft and cause temporary malfunction of several CCD cameras (see the "snowstorms" in Figures 1, 2 and 3).

Such big events and event sequences do not occur frequently. Since the beginning of regular registrations by NOAA in 1976 there had been only three events with slightly higher proton fluxes: on October 19, 1989 (Reeves et al., 1992), on March 24, 1991, and on November 4, 2001 (according to NOAA records, see http://goes.ngdc.noaa.gov/data/ParticleEvents.txt). Note that these three big SEP events were associated with flares of importance X13, X9, and X1, respectively, while at some much bigger flares (at similarly central positions on the Sun's disk) the SEP fluxes remained rather low.

The acceleration of particles to such high energies on time scales of seconds or minutes as well as their propagation through space is still not well understood, and active research is going on. The interested reader may wish to study more detailed reviews than the present one can offer, e.g., Kunow et al. (1991); Reames (1999, 2001, 2002); Tylka (2001); Kahler (2001a), and of other authors such as Kallenrode, Lee, Lin, Mason.

\subsection{SEPs: protons and other ions}

The time profile of the strong SEP proton flux event of November 4, 2001 (from Reames, 2004) is shown in Figure 22. After a steep flux increase within minutes after flare onset, the fluxes in the low energy bands remain about constant for several hours while for higher energies the fluxes gradually drop, i.e., the energy spectra become steeper. Later on, a general flux increase in all channels culminates in a distinct peak that exactly coincides with the passage of the associated shock wave at the observing spacecraft. Even relativistic protons with energies of more than $510 \mathrm{MeV}$ peak at the shock that is driven by a sufficiently fast CME coming from the region near

Living Reviews in Solar Physics

http://www. livingreviews.org/lrsp-2006-2 

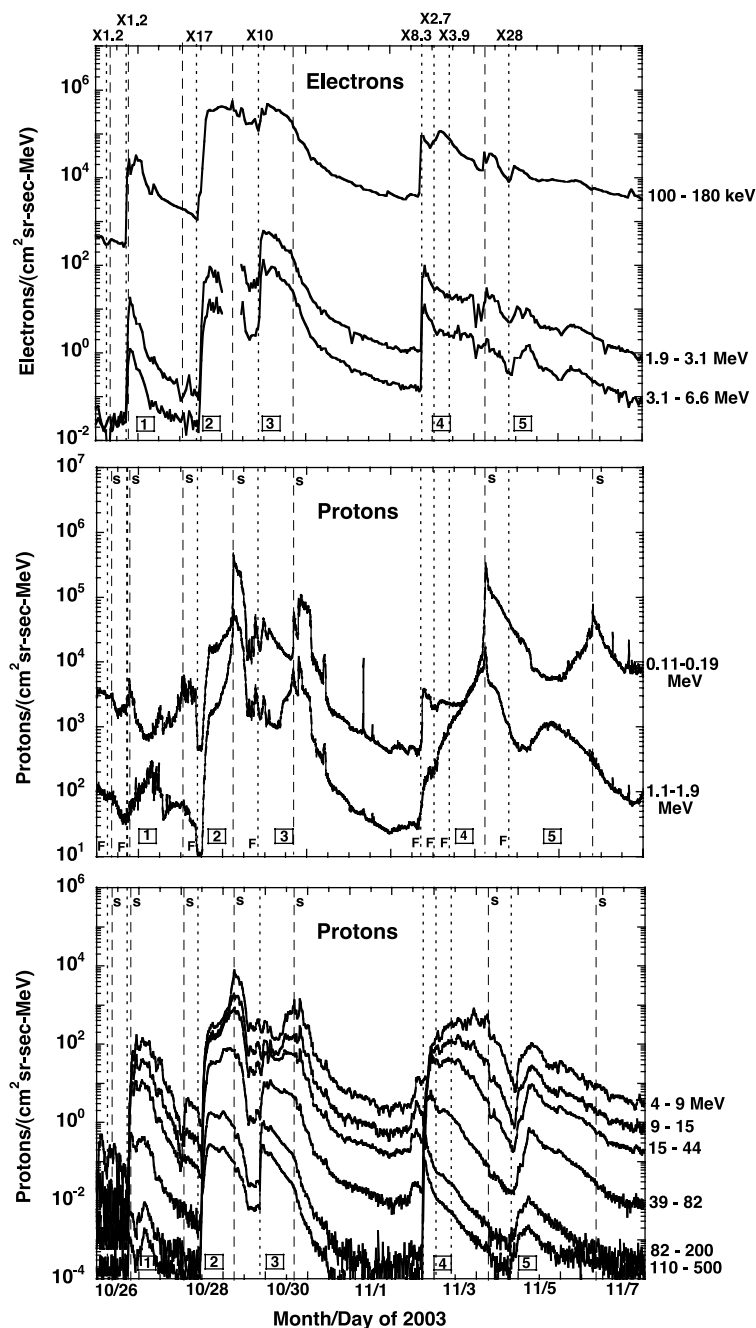

Figure 21: Time history of energetic protons and electrons during the period from October 26, 2003 to November 7, 2003. The top panel shows electron data from EPAM/ACE (top trace) and PET/SAMPEX $(1.9$ to $6.6 \mathrm{MeV})$. The SAMPEX points are averaged over separate polar passes, including only data obtained at invariant latitudes $>75^{\circ}$. It is possible that the intensities shown near the end of 29 October through 30 October (SEP event 3) are overestimated because of background contributions. The middle panel shows low-energy proton data from ACE/EPAM and the bottom panel includes protons measured by GOES-11 in six different energy intervals. The occurrence of X-class flares (obtained from NOAA) are indicated by dotted vertical lines with the intensity labeled above each line. Interplanetary shocks are indicated by dashed vertical lines labeled by an "s". Major proton events during this interval are labeled 1 to 5. From Mewaldt et al. (2005). 
the Sun's disk center. Generally, the peak proton intensity at shock passage was found to be correlated best with the initial speed of the associated CME (Kahler et al., 1984; Kahler, 2001b). Only the fastest $\sim 1 \%$ of CMEs produce significant SEP effects. The longitude difference between the CME source and the observer as well as the angular extension of the shock wave may also affect the local SEP fluxes.

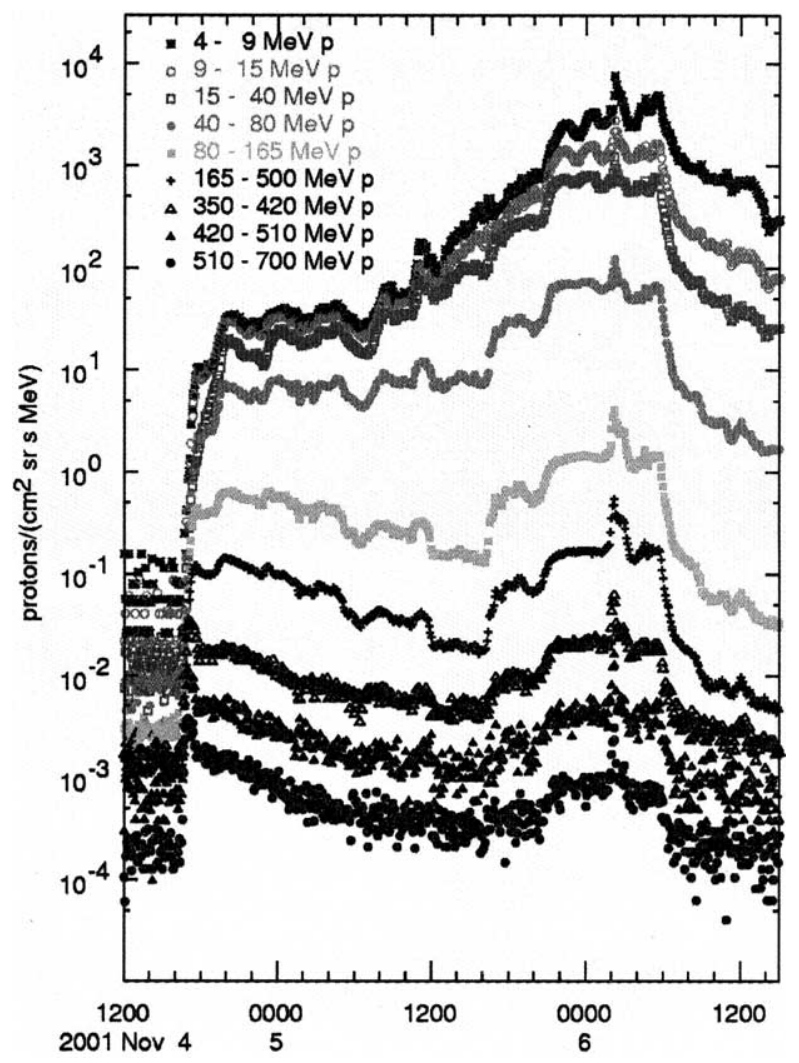

Figure 22: Time profiles of the strong SEP proton flux event of November 4, 2001. The peak at the time of shock passage is clearly defined early on November 6, even at proton energies as high as $510-700 \mathrm{MeV}$. From Reames (2004).

The realization of large SEP events being driven by CME shock waves rather than by solar flares meant a major paradigm change in the early 1990s (see Reames et al., 1996, and references therein). After all, SEPs may become a tool to probe the shock and topology of the shock. By comparing complete intensity-time profiles of SEPs from several spacecraft one may obtain selfconsistent models of the evolving structures.

Figure 23 (from Reames et al., 1996) shows the time profiles of SEP fluxes as seen by observers viewing a large CME shock from 3 different longitudes. The left hand panel shows what an observer located east of the CME will see. Since he is magnetically well connected to some point along the nose shock early on, he will notice a rapid rise of SEPs. The decline is due to the fact that he will be connected with increasingly weaker parts of the eastern shock flank. Another observer stationed near the central meridian will see a slower initial rise since he is connected with the western shock flank. Then, due to a large extended shock front, he is always well-connected until the shock passes: thus, he sees flat profiles (middle panel). An observer located on the western flank of the

Living Reviews in Solar Physics

http: //www. livingreviews.org/lrsp-2006-2 
CME is poorly connected with the distant western shock flank until after the shock passes. Then he is connected (on the backside of the shock) with the powerful nose of the shock and encounters high SEP fluxes for quite some time.

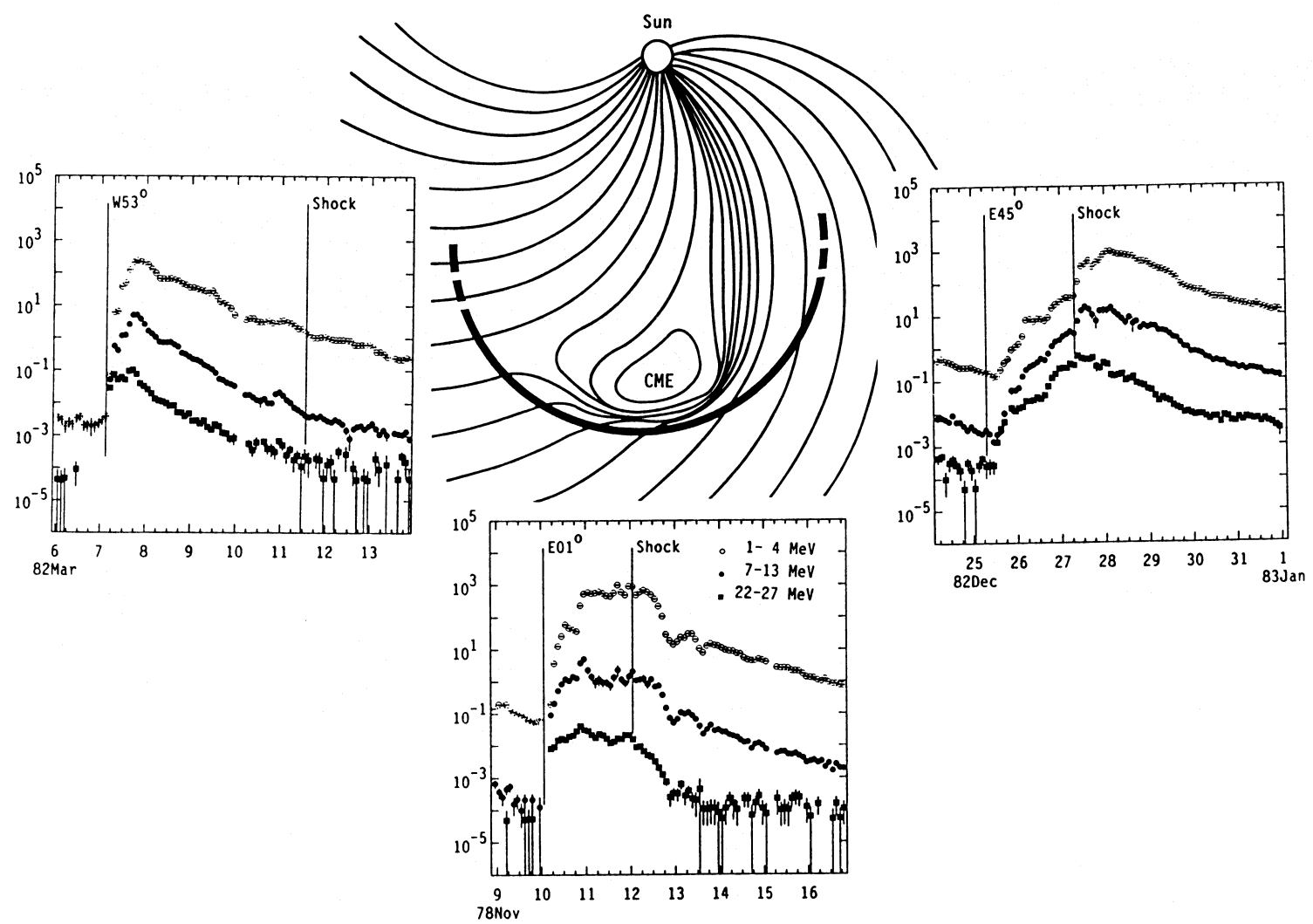

Figure 23: Typical intensity-time profiles for protons of 3 different energies are shown as seen by observers viewing a large CME-driven shock from 3 different longitudes indicated. The observer seeing a western event (left hand panel) is well connected to the nose of the shock early and sees rapid rise and decline. Another observer near central meridian (middle panel) is well-connected until the shock passes; thus, he sees flat profiles. The observer viewing an eastern event is poorly connected until after the local shock passes, when he is on the field lines that connect him to the powerful nose of the shock (from behind). From Reames et al. (1996)

It is now widely agreed that SEPs come from two different sources with different acceleration mechanisms working: The flares themselves release impulsive events while the CME shocks produce gradual events (see the terminology discussion by Cliver and Cane, 2002). The SEPs from flares often have major enhancements in ${ }^{3} \mathrm{He} /{ }^{4} \mathrm{He}$ and enhanced heavy ion abundances, because of resonant wave-particle interactions in the flare site. The ions usually have usually very high ionization states. However, the most intense SEP events, also with the highest energies, are produced by CME driven shocks. These SEPs reflect the abundances and ionization states of the ambient coronal material.

The terms impulsive and gradual originally came from the time scales of the associated X-ray events, but nowadays they are applied to distinguish the time scales of SEP events. In fact, the time profiles of impulsive and gradual SEP events look rather different as is shown in Figure 11 of Reames (1999). The gradual event is due to an erupting filament as part of a CME, with 
no accompanying flare. The impulsive events were associated with several impulsive flares, but without any CME signatures. The gradual event is dominated by protons, with a small peak at shock passage. The smooth and extended time profile comes from continuous acceleration at the moving CME shock. In the impulsive event the electron fluxes are higher than those of the protons and those of the gradual event, respectively. The comparatively short duration of the impulsive event is determined by scattering of the particles as they traverse interplanetary space.

The differentiation between these two types of SEP events is now rather straightforward and unique. Some statistical analyses revealed interesting facts. Reames (1999) compared the "source longitude" of the associated flares for the two types. The distribution of gradual events was found to be almost uniform across the face of the Sun. Determination of a "source longitude" is complicated since many gradual SEP events apparently originated from behind the Sun's limb, and many CMEs driving gradual SEP fluxes were not associated with flares. After all, there is no doubt about a pretty uniform distribution. In contrast, the impulsive event sources are clearly concentrated in the western half of the Sun with a surprisingly sharp peak at W60. That is about the source longitude of the "average" Parker spiral that connects the Earth to the Sun. Apparently, these impulsive SEPs are injected right into and contained well within "their" flux tube. They do not have much chance to escape to neighboring field lines. The comparison of the distributions indicates that the broad distribution of gradual events can not result from cross-field diffusion, since there is no reason why this same process would not also broaden the distribution of impulsive events. The broad distribution of gradual events suggests the existence of large-scale shock waves that can easily propagate across field lines. The long-standing problem "How can flare-accelerated particles be transported to the often very distant field lines where they are observed?" that had puzzled whole generations of scientists (see, e.g. Kunow et al., 1991) can finally be considered solved.

There is an important implication with respect to space weather effects: The direct injection of impulsive SEPs affects only a narrow regime in space. But the shock fronts driven by CMEs can extend over large spatial angles and thus can fill them with high fluxes of SEPs (Simnett, 2003). In particular, the very big and fast events produce the highly relativistic and most dangerous particles and spread them almost all around the Sun, covering nearly the whole heliosphere.

There is a vast literature on the important issue of elemental abundances in SEP fluxes. Most impulsive flares show not only substantial enhancements of the ${ }^{3} \mathrm{He} /{ }^{4} \mathrm{He}$ ratio but also enhanced heavy ion contents, as compared to oxygen (Reames and $\mathrm{Ng}, 2004$ ). It is thought that these anomalies contain information on the acceleration and propagation processes. These aspects will not be addressed further in this review. The interested reader can easily find (e.g., by searching the ADS) relevant papers by authors such as Reames, Klecker, Cane, Mason.

\subsection{SEPs: electrons}

The fluxes of non-thermal electrons as products of solar transient processes are very variable and hard to predict (for a general review see Lin, 1985). Apparently, there is a variety of processes to produce these electrons: 1) The energy spectra extend smoothly to the suprathermal range, down to $2 \mathrm{keV}$, indicating that these events originate high in the corona (Potter et al., 1980). 2) Electrons accelerated at flare sites with a very wide energy spectrum that cause the radio type III bursts as discussed above (Alvarez et al., 1972). 3) Electrons accelerated at the propagating interplanetary shock waves, both upward and downward, leading to the characteristic "herringbone" pattern of radio spectra shown in Figure 19 (Mann and Klassen, 2005).

At low energies below a few tens of $\mathrm{keV}$, the observed electron fluxes may be a mixture of all the mentioned sources. That is probably the reason why spectra in this energy range are so variable and often show several breaks and kinks (see, e.g. Lin, 1985). At higher energies, above a few $\mathrm{MeV}$, the situation changes. Evenson et al. (1984) found that most events with high fluxes of 5 to

Living Reviews in Solar Physics

http://www. livingreviews . org/lrsp-2006-2 
$50 \mathrm{MeV}$ electrons appear to have been produced by flares which also produced observable fluxes of Gamma-rays. These events are never accompanied by strong interplanetary shocks which therefore are not a source of electron acceleration above a few $\mathrm{MeV}$. It is concluded that the variable nature of the interplanetary particle events must reflect some fundamental but variable property of the shocks - possibly their direction of propagation.

For the very large events, electrons with energies up to $100 \mathrm{MeV}$ have been observed (Simnett, 1974; Moses et al., 1989). The energy spectra are usually rather hard, i.e., with power law indices of 3 and higher. For some events, spectral steepening above a few MeV is observed (Lin et al., 1982). It is not clear yet whether the energy spectrum extends beyond $100 \mathrm{MeV}$.

There are several open questions concerning the origin and propagation of energetic electrons from flares and shocks. This is illustrated by the recent ongoing controversy about "delayed injection". The well-known type III burst producing electrons with their typical energies below $30 \mathrm{keV}$ are also directly observed near the Earth. However, some near-relativistic electron events (energies above $30 \mathrm{keV}$ ) were released up to 30 minutes later in comparison to the type III radio emission onset. For the origin of this delay three explanations are being debated: 1.) The delayed electrons come from coronal shocks, like the herringbones at type II radio bursts (Krucker et al., 1999; Klassen et al., 2002; Haggerty and Roelof, 2002). 2.) The delayed electrons originate at reconnection site behind the shock front, and the CME shock itself plays only a minor role (Laitinen et al., 2000; Klein et al., 2001; Maia and Pick, 2004). 3.) All electrons originate from a single population, and the delay is due to propagation effects across magnetic field lines (Cane, 2003). Klassen et al. (2005) analyzed the October 28, 2003 flare and found three separate stages of injection. After some detailed analysis and discussion they state: "... the association of the two delayed injections with solar events is not well understood."

In this context, another potentially relevant issue should at least be mentioned. Several authors recently addressed the appearance of a local minimum and maximum of the local Alfvén velocity in the middle of the corona and at $4 R_{\mathrm{s}}$, respectively. That would have effects on the formation of shocks in the corona and interplanetary space as well as the temporal behavior of the associated energetic particle events (Gopalswamy et al., 2001a; Mann et al., 1999, 2003; Vršnak et al., 2004; Warmuth and Mann, 2005).

\subsection{SEPs: neutrons}

At the biggest flares protons are accelerated to energies of several GeV. Upon interaction with other atoms they cause nuclear reactions that release Gamma-rays and relativistic neutrons. Chupp et al. $(1982,1987)$ reported the first direct detection of solar neutrons from a satellite-borne instrument following an impulsive solar flare. Identification of the n-p capture line at $2.223 \mathrm{MeV}$ observed at energetic flares confirms the existence of flare-associated neutrons. Combining these observations, it is possible to study the solar neutron energy spectrum from $\sim 1 \mathrm{MeV}$ to several GeV. Ramaty et al. (1983) pointed out that this allows us to investigate the time history of particle acceleration in solar flares and to determine the total number and the energy spectrum of the accelerated ions up to the highest energies.

Due to the absence of charge, solar neutrons can reach the Earth fully unhindered, provided their lifetime is long enough. That is the case only for relativistic neutrons. However, it is difficult to differentiate solar neutrons from those neutrons that are generated in the Earth's atmosphere. In fact, when a high flux of relativistic SEPs strikes the Earth's atmosphere, spallation of atmospheric atoms sets on that lets nuclear byproducts cascade down such that enhanced neutron fluxes reach the ground. For monitoring these so-called ground level events (GLEs), a network of neutron monitors has been installed all over the globe, e.g., the "Spaceship Earth" observing network (Bieber et al., 2004). It provides an effective means of studying the angular distribution and energy spectrum of the most energetic SEPs. The problem is that these SEPs and the solar 
neutrons are generated simultaneously and, thus, should arrive at the Earth almost simultaneously as well.

During the Halloween events three GLEs were observed. The one on October 28 was of particular interest: About 8 minutes before the main GLE seen by several stations, the one in Tsumeb, South Africa saw a very weak but distinct neutron flux (Bieber et al., 2005), simultaneously with the SONG neutron detector on the CORONAS-F spacecraft (Miroshnichenko et al., 2005), as is shown in Figure 24. Assuming that the first solar neutrons were emitted at the onset of the most conspicuous electromagnetic emissions from the Sun, the travel time to Earth allows an estimate of the neutrons' energy of 400 to $500 \mathrm{MeV}$.

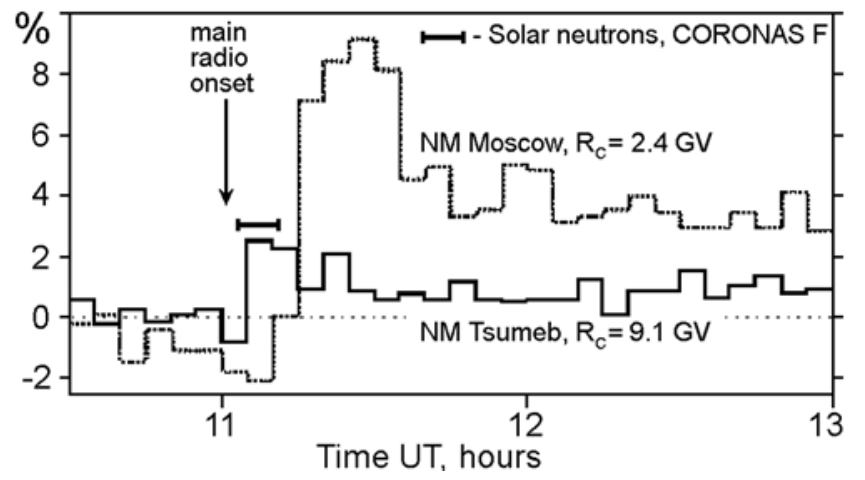

Figure 24: Increase profiles of the GLE observed with 5-min averages by Tsumeb and Moscow neutron monitors. The vertical arrow indicates the start of bright radio emission at 1102 UT. The horizontal bar marks the time when neutrons were detected by the SONG instrument at CORONAS-F spacecraft. From Miroshnichenko et al. (2005).

\subsection{The acceleration of SEPs}

The sudden release of huge amounts of energy in flares and CMEs is an issue with many unsolved problems. One of them concerns the acceleration of electrons and ions to such extremely high energies. From the manifold observations discussed above we have already many hints about the mechanisms involved. On the other hand, these observations will help to decide on whether a theoretical model is correct or not. In this review, I will not elaborate on this multi-faceted subject and rather leave it to other reviews to come. In the meantime, the interested reader may wish to study the articles in the book by Klein (2003), in particular the one by Kliem et al. (2003).

\subsection{SEPs and space weather}

The SEP fluxes from flares and CME shocks affect space weather in two main aspects. 1) The relativistic proton fluxes can lead to dangerous radiation doses to unprotected technical systems and astronauts. 2) The fluxes of energetic protons impact the Earth's middle atmospheric polar caps by producing enhanced ionizations, excitations, dissociations of atmospheric atoms and molecules. That causes, e.g., ozone depletions in the polar upper stratosphere (Jackman et al., 2005; Rohen et al., 2005). There are some other relevant articles in the special editions of Geophys. Res. Lett. and J. Geophys. Res., see http://www.agu.org/journals/ss/VIOLCONN1/. 


\section{Coronal Mass Ejections (CMEs)}

Since Carrington's accidental flare observation in 1859, it was established that geomagnetic storms are linked to solar activity and come in a very irregular fashion. More than one hundred years later, a different category of highly energetic transient events on the Sun was discovered (Brueckner, 1974; Gosling et al., 1974), in the course of which huge amounts of gas are ejected from the Sun into interplanetary space. After some years of unclear terminology, the use of the name coronal mass ejections (CMEs) became common. Their pronounced significance for the Earth was soon revealed. Although the discovery of CMEs was made only some 30 years ago, a very vast literature has been piled up, and the flood of new publications is still growing. A great number of review articles and books is available to the interested reader (see, e.g., Hildner, 1977; MacQueen, 1980; Wagner, 1984; Schwenn, 1986; Hundhausen, 1987; Kahler, 1992; Crooker et al., 1997; Hundhausen, 1997; Low, 2001; Webb et al., 2001; Gopalswamy, 2004; a comprehensive book on CMEs was issued by ISSI in 2006).

Before going into details, I consider it worthwhile to repeat the definition of a CME (Hundhausen et al., 1984, see also Schwenn, 1996):

We define a coronal mass ejection (CME) to be an observable change in coronal structure that 1) occurs on a time scale of a few minutes and several hours and 2) involves the appearance (and outward motion) of a new, discrete, bright, white light feature in the coronagraph field of view.

Note that this definition does not specify the origin of the "feature", nor its nature, be it the ejecta themselves or the effects driven by them.

CMEs cause gigantic plasma clouds to leave the Sun, which then drive large-scale density waves out into space. In the animation of Figure 25 a typical CME as observed on February 27, 2000, by the coronagraph LASCO C3 onboard the SOHO spacecraft is shown. This dramatic CME (often termed the "light bulb CME", for obvious reasons) originated from an erupting mid latitude filament in the north-east quadrant of the Earth-facing Sun disk, and it probably was pointed well-above the Earth. The apparent position angle of the centroid of about $30^{\circ}$ (measured anticlockwise from the north pole) is probably due to a projection effect. Note how well the $60^{\circ}$ cone angle and the general shape are maintained during the whole 12 hour passage through the LASCO field of view. This maintained "self-similarity" is characteristic for most CMEs (Low, 2001).

In Figure 26, and in the animated Figures 27, 28, and 29, I show a collection of CME images that may indicate the huge variety of CME shapes. However, much of that variety is due to projection effects. CMEs occurring close to the disk center often appear to surround the occulting disk of the coronagraph and are thus known as halo CMEs (Howard et al., 1982), CMEs with apparent widths between $120^{\circ}$ and $360^{\circ}$ are known as partial halos. Halos can be front-sided or back-sided, and for differentiation simultaneous disk observations are required. A "normal" CME, seen above the limb with an angular width of, say, $60^{\circ}$, will appear as a halo CME or partial halo CME when oriented along the Sun-Earth-line (both: towards to or away from Earth) or some $40^{\circ}$ off that line, respectively.

\subsection{CME properties}

CMEs are characterized by speed, angular width, and a central position angle in the sky plane. Measured front speeds range from a few km/s (near the Sun) to nearly $3000 \mathrm{~km} / \mathrm{s}$ (Howard et al., 1985; St Cyr et al., 2000; Gopalswamy, 2004). The apparent angular width of CMEs ranges from a few degrees to more than $120^{\circ}$, with an average value of about $50^{\circ}$ (partial and full halos excluded). Note that width and source latitude are severely affected by projection effects (Burkepile et al., 2004; Cremades and Bothmer, 2004).

The total mass ejected in CMEs ranges from some $10^{13} \mathrm{~g}$ to a few $10^{16} \mathrm{~g}$, the total energy 


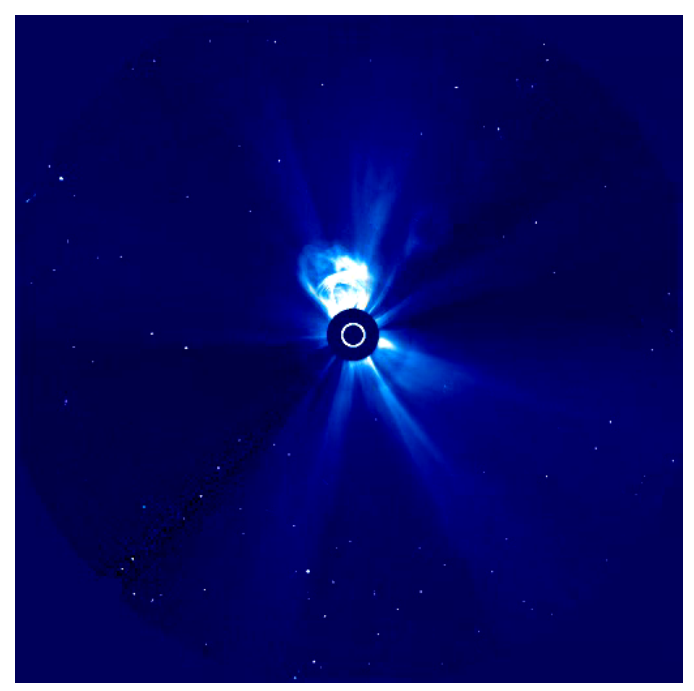

Figure 25: Still from a movie showing The "light bulb" CME, as observed by the LASCO C2 and C3 coronagraphs onboard SOHO. The animation covers a time period of about 24 hours, starting on February 27, 2000, 00:18 UT. (To watch the movie, please go to the online version of this review article at http: //www . livingreviews .org/lrsp-2006-2.)

(kinetic plus potential energy) from $10^{27} \mathrm{erg}$ to some $10^{33} \mathrm{erg}$, with averages of $1.4 \times 10^{15} \mathrm{~g}$ and $2.6 \times 10^{30} \mathrm{erg}$, respectively (Vourlidas et al., 2002; Gopalswamy, 2004).

Yashiro et al. (2004) have assembled a huge catalog of more than 10,000 CMEs (status 2006, still counting), all discovered upon visual inspection of data from the SOHO instruments. For each event they present important information (e.g., images taken by the EIT and LASCO instruments, animations, difference images, and some important characteristic properties), and make it easily accessible to the science community through the public website http://cdaw.gsfc.nasa.gov/ CME_list/. The catalog contains also time-height diagrams which are evaluated for the fastest section of the outermost front. Thus, values for speed and acceleration of these plane-of-sky projections are derived. The diagram for the light bulb CME is shown in Figure 30, right panel. In this case all points can be fitted pretty well by a simple straight line, indicating a constant speed value of about $570 \mathrm{~km} / \mathrm{s}$. In other cases, there is acceleration seen in the inner range, usually up to about $10 R_{\mathrm{s}}$, often followed by deceleration in the outer range, from about $15 R_{\mathrm{s}}$ on (see example in Figure 30, left panel). The single acceleration values listed in the catalog by Yashiro et al. (2004) are thus at least doubtful.

There are other attemts to identify CMEs. Note, e.g., the automated CME detection scheme as described by Robbrecht et al. (2009), cf. http://sidc.oma.be/cactus/.

The apparent 3-part structure of the light bulb CME (a bright outer loop, followed by a dark void and finally by a bright kernel) is particularly noteworthy. This kind of topology is very common (Hundhausen, 1987). The large number of CMEs observed since their discovery in 1973 has allowed statistical analyses of their properties. The results are sometimes controversial, because of different evaluation schemes that often are biased by subjective judgments. I refer the reader to papers by Hundhausen et al. (1984); Howard et al. (1985); St Cyr et al. (2000); Gopalswamy (2004).

Some authors claim that there are two (or more) kinds of coronal mass ejections (e.g. Sheeley Jr et al., 1999; Srivastava et al., 1999a,b; Švestka, 2001; Moon et al., 2002): (1) Gradual CMEs, with balloon-like shapes, accelerating slowly and over large distances to speeds in the range 300 to $600 \mathrm{~km} / \mathrm{s}$, and (2) Impulsive CMEs, often associated with flares, accelerated already low down to

Living Reviews in Solar Physics

http: //www. livingreviews.org/lrsp-2006-2 

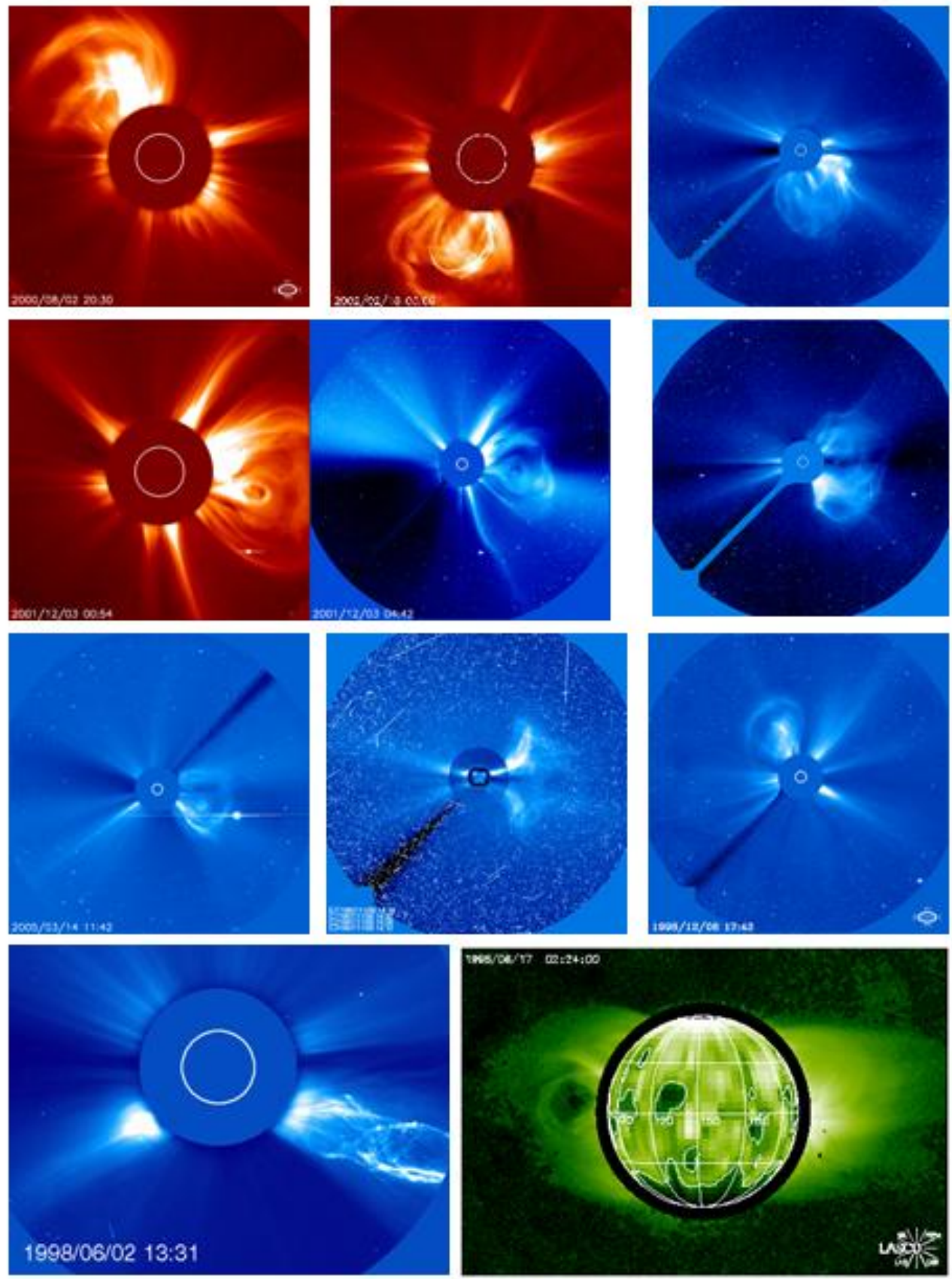

Figure 26: Examples of CMEs as observed by the LASCO C2 and C3 coronagraphs on SOHO. 


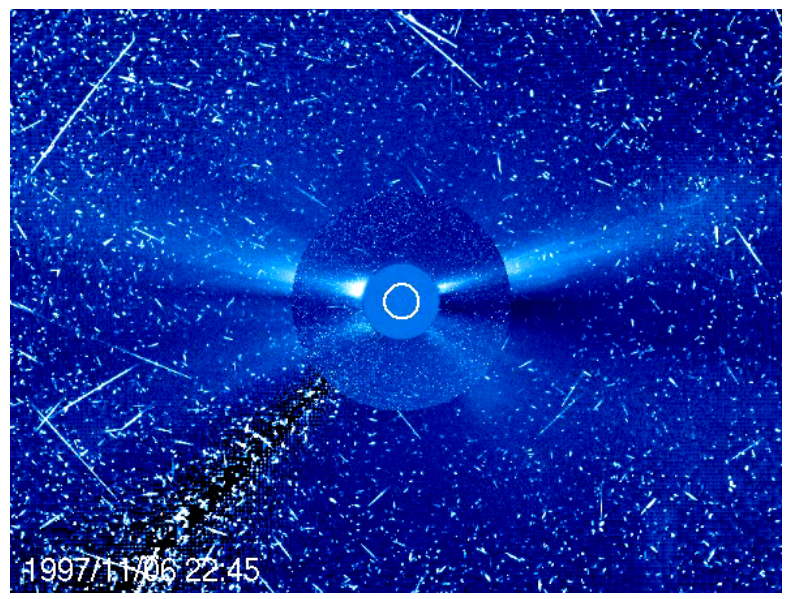

Figure 27: Still from a movie showing The limb CME of November 5, 1997, as seen by the LASCO C2 and $\mathrm{C} 3$ coronagraphs on SOHO. (To watch the movie, please go to the online version of this review article at http://www.livingreviews.org/lrsp-2006-2.)

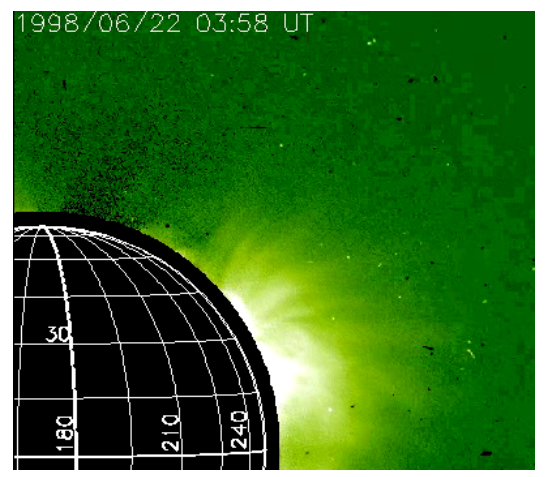

Figure 28: Still from a movie showing The balloon type CME of June 21, 1998, as seen by the LASCO C1 coronagraph on SOHO. (To watch the movie, please go to the online version of this review article at http: //www . livingreviews . org/lrsp-2006-2.) 


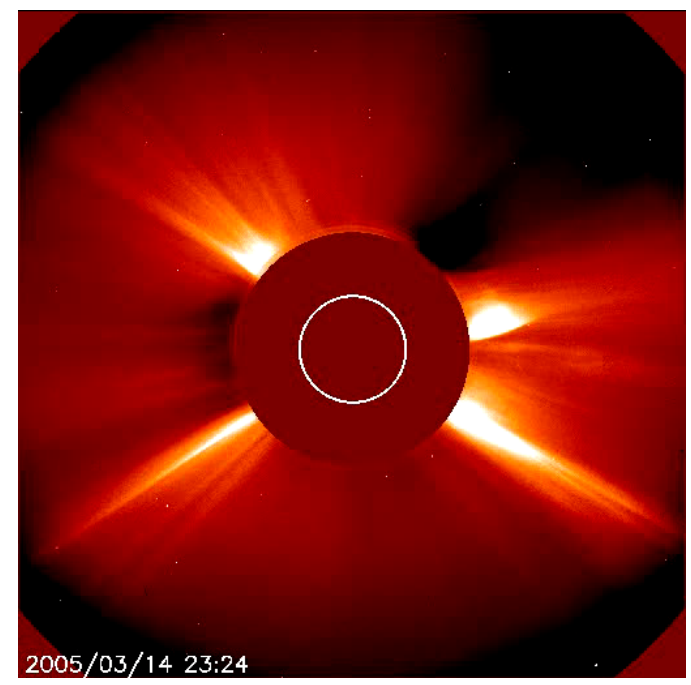

Figure 29: Still from a movie showing The limb CME of March 14, 2005, as seen by the LASCO C2 coronagraph (To watch the movie, please go to the online version of this review article at http://www. livingreviews.org/lrsp-2006-2.)
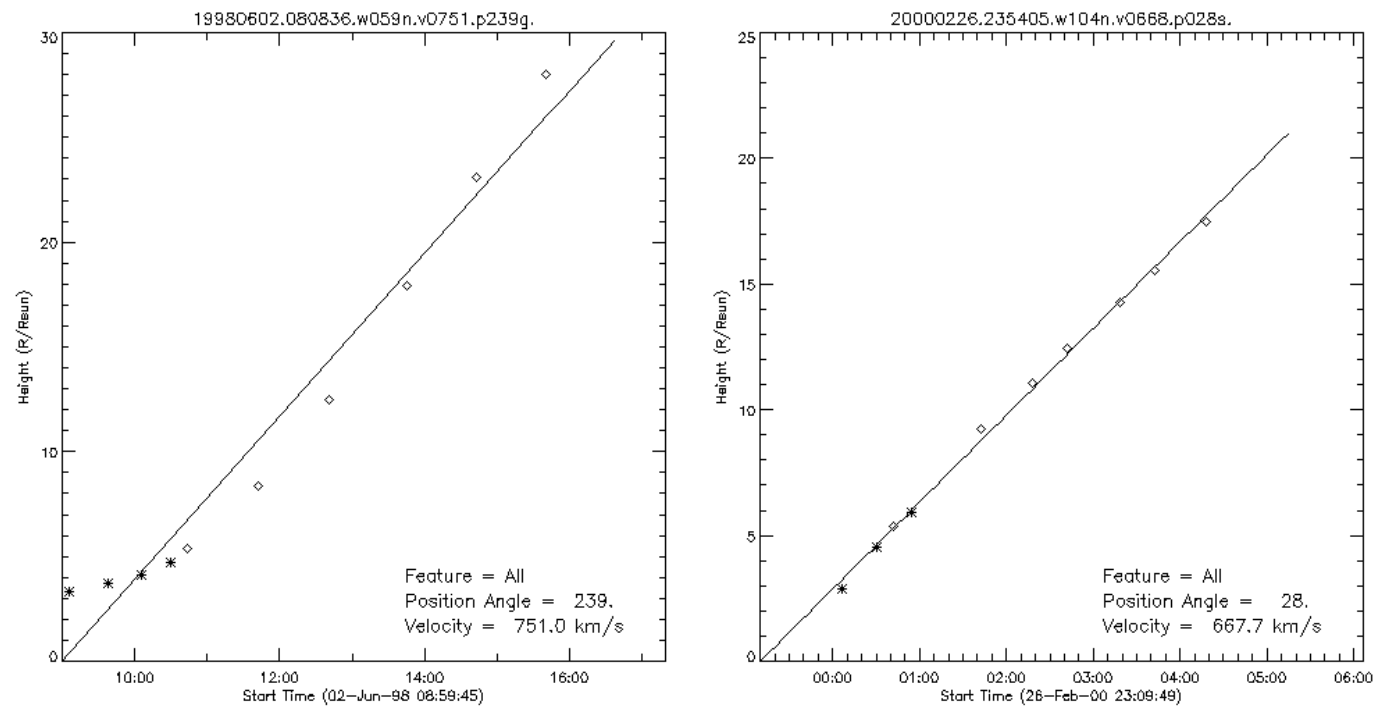

Figure 30: Left panel: Time-height diagram for the CME of June 2, 1998. Right panel: Time-height diagram for the light bulb CME, as derived by Yashiro et al. (2004), see http://cdaw.gsfc.nasa.gov/ CME_list/) 
extreme speeds (sometimes more than $2000 \mathrm{~km} / \mathrm{s}$ ). It is not clear yet whether these are really fundamentally different processes or whether they represent just the extrema of an otherwise continuous spectrum of CME properties.

Zhang et al. (2001, 2004) described the initiation of CMEs in a three-phase scenario: the initiation phase, the impulsive acceleration phase and the propagation phase. The initiation phase (taking some tens of minutes) always occurs before the onset of an associated flare, and the impulsive phase coincides well with the flare's rise phase. The acceleration ceases with the peak of soft X-ray flares. It is interesting to notice that some of the theoretical CME models begin to postulate different phases of acceleration (see, e.g. Chen and Krall, 2003).

Right at the launch time of several CMEs, Kaufmann et al. (2003) discovered rapid solar spikes at radio submillimeter wavelengths that might be representative of an early signature of CME onset. The role of some other observed processes is also still unclear: coronal "dimmings" (Hudson et al., 2003), Moreton waves (named after their discoverer, see Moreton (1960), also Thompson, 2000), EIT waves (named after the EIT instrument on SOHO that made them visible, see Thompson et al., 1998), the various types of radio bursts (Reiner et al., 2001), coronal inflow (Wang et al., 1999; Sheeley Jr and Wang, 2002; Tripathi et al., 2005).

Most CMEs are originating near the heliographic equator (Howard et al., 1985; St Cyr et al., 2000). Sometimes CMEs are seen at very high latitudes (for example the light bulb CME shown in Figure 25). Usually these are CMEs originating at mid latitudes but directed near the Sun-Earth line, such that in projection they look as if they were poleward pointed (Burkepile et al., 2004). Cremades and Bothmer (2004) corrected this projection effect for some 200 CMEs observed between 1996 and 2002 and determined the "true" center latitudes at several solar radii from the Sun. At solar minimum, they clearly peak at the solar equator. But their source regions as determined near the surface from EIT images are centered in two belts at around $25^{\circ}$ northern or southern latitude. That means that these CMEs must have been deflected from their mid-latitude sources towards the solar equator. Cremades and Bothmer (2004) found that the deflection is proportional to both: the proximity and the size of coronal holes. At times of high solar activity, in absence of the big polar coronal holes, there was no net deflection found. The latitudinal distribution of source regions and CME center latitudes were generally broader, but high latitude CMEs were observed rarely even then.

The shapes of the vast majority of CMEs appear to be consistent with a nearly perfect cross section. Indeed, halo CMEs moving exactly along the Earth-Sun line exhibit generally a circular and smooth shape (see examples in Figures 31, 32, 33). This observation is rather surprising in that CMEs result from the eruption of basically 2D elongated filament structures (for further discussion see the review by Schwenn, 1986 and Cremades and Bothmer, 2004). Thus, the apparent lateral expansion of CMEs can be considered independent of the viewing direction.

Making use of that, Schwenn et al. (2005) point out that the speed of lateral CME expansion is the only CME parameter that can be uniquely determined for any CME, be it on the limb or pointed along the Earth-Sun line, on the front as well as on the back side. They selected a representative subset of 57 unique limb CMEs. For those the true radial speed $V_{\text {rad }}$ and the expansion speed $V_{\text {exp }}$ measured across the full CME width in the direction perpendicular to $V_{\text {rad }}$ were determined. A striking correlation was found:

$$
V_{\text {rad }}=0.88 V_{\text {exp }}
$$

with a correlation factor of 0.86 .

This correlation holds for the slow CMEs as well as for the fast ones, for the narrow ones as well as the wide ones. This means that the quantity $V_{\exp }$ can actually be used as proxy for the frontal speed that is most often inaccessible because of projection effects. Schwenn et al. (2005)

Living Reviews in Solar Physics

http://www. livingreviews . org/lrsp-2006-2 

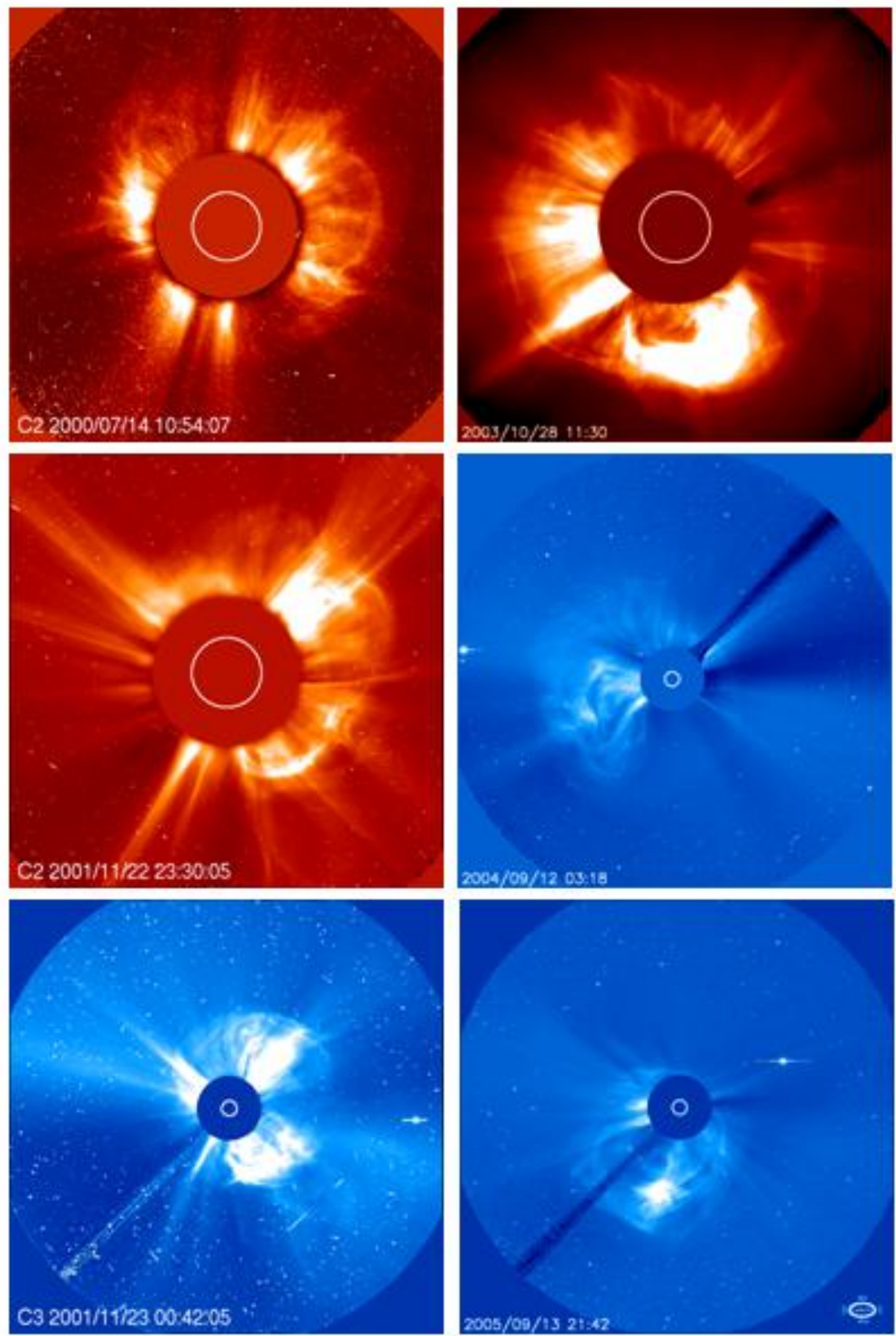

Figure 31: Examples of halo CMEs as observed by the LASCO C2 and C3 coronagraphs on SOHO. 


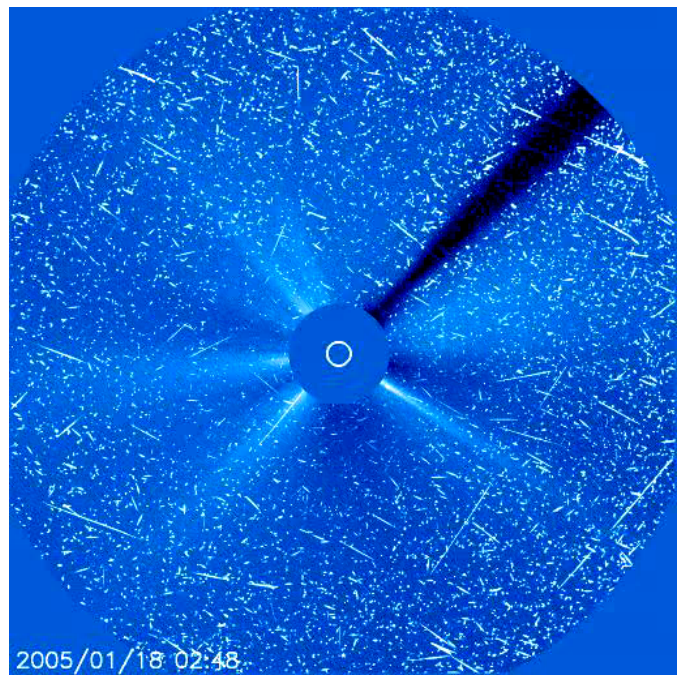

Figure 32: Still from a movie showing A series of halo CMEs, observed by the LASCO C3 coronagraph on SOHO from January 14 to 18, 2005. (To watch the movie, please go to the online version of this review article at http://www.livingreviews.org/lrsp-2006-2.)

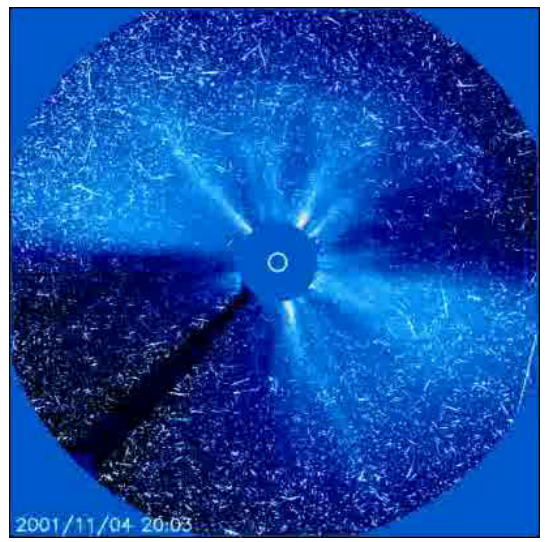

Figure 33: Still from a movie showing The halo CME of November 4, 2001, as seen by the LASCO C3 coronagraph on SOHO. The loudness of the sound represents the intensity of SEPs during the event. This and other sonifications were prepared by a group of talented musician scientist at SSL in Berkeley http://cse.ssl.berkeley.edu/impact/sounds.html (To watch the movie, please go to the online version of this review article at http://www.livingreviews.org/lrsp-2006-2.) 
demonstrated that the expansion speed $V_{\exp }$ can be used as a rather simple though empirical tool for predicting the travel times of CME disturbances to the Earth (see Section 5.3).

After all, we have to admit that some fundamental questions about CMEs are still unsolved. Most importantly: What causes a CME to erupt in the first place? The situation is similarly embarrassing as for flares. Many researchers around the world are intensely tackling this problem. However, the essential ingredients for $\mathrm{CME}$ onset are not yet identified. Some candidates: the proximity of a CME site to coronal holes (Bravo et al., 1999), magnetic shear (Mikić and Linker, 1997), filament helicity (Martin, 2003; Rust, 2003), sigmoids (Rust and Kumar, 1996; Moore et al., 2001).

In order to disentangle the various processes around CME initiation new observations with significantly better resolution (spatially and in time) and even supported by spectroscopic diagnostics are needed, as was demonstrated by Innes et al. (2001) and Balmaceda et al. (2003). There is hope that the new Hinode and SDO missions with their excellent instruments will allow major progress in the field.

\subsection{Interplanetary counterparts of CMEs: ICMEs}

The fast CMEs often drive large-scale density waves out into space which eventually steepen to form collisionless shock waves, similar to the bow shock in front of the Earth's magnetosphere. The shock front is the outer boundary of a plasma sheath (see, e.g. Tsurutani et al., 1988) that results from compression, deflection, and heating of the ambient solar wind by the ensuing ejecta. The sheath may contain substantial distortions of the interplanetary magnetic field due to field line draping (McComas et al., 1988) around the ejecta cloud pressing from behind. It has become common to summarize all these CME driven effects under the term ICME (for interplanetary counterparts of CMEs, see terminology discussion by Burlaga, 2001 and Russell, 2001).

The ejecta themselves (called "piston gas" or "driver gas" in earlier papers) have properties that differ radically from those of the ambient solar wind. At first, the ejecta are often separated from the sheath plasma by a tangential discontinuity. Their very different origin is discernible from their different elemental composition (Hirshberg et al., 1971), ionization state (Bame et al., 1979; Schwenn et al., 1980; Henke et al., 1998; Rodriguez et al., 2004), temperature depressions (Gosling et al., 1973; Montgomery et al., 1974; Richardson and Cane, 1995), cosmic ray intensity decreases ("Forbush decreases", see, e.g. Cane et al., 1994), the appearance of bi-directional distributions of energetic protons and cosmic rays (Palmer et al., 1978) and supra-thermal electrons (Gosling et al., 1987). In many ejecta, major overabundances of Helium are observed, up to $30 \%$, as was first noted by Hirshberg et al. (1971). This indicates that ejecta material originates from low layers in the solar atmosphere, where gravitational stratification allows substantial enrichment of heavy ions.

For about one third of all shocks driven by ICMEs, the succeeding plasma exhibits to an in situ observer the topology of magnetic clouds (Burlaga et al., 1981), see reviews by, e.g., Gosling (1990); Burlaga (1991); Osherovich and Burlaga (1997). Smooth rotation of the field vector in a plane vertical to the propagation direction, mostly combined with very low plasma beta, i.e., low plasma densities and strong IMF with low variance give evidence of a flux rope topology (Marubashi, 1986; Bothmer and Schwenn, 1998) of these magnetic clouds. This is consistent with the concept of magnetic reconnection processes (we might better call them "disconnection" processes) in coronal loop systems in the course of prominence eruptions at the Sun (Priest, 1988). It is true though that the boundaries of magnetic clouds are often difficult to identify (Goldstein et al., 1998; Wei et al., 2003).

Most of these ICME signatures can be seen in the event shown in Figure 34. Usually, only a subset of the criteria for identifying ejecta is encountered in individual events, and to this day a trained expert's eye is needed to tell what is ejecta and what not. The situation is additionally 
complicated by the class of very slow CMEs found to take off more like balloons rather than as fast projectiles (Srivastava et al., 2000). After many hours of slow rise, they finally float along in the ambient slow solar wind. Naturally, they do not drive a shock wave. Only in rare cases, a few of their ejecta signatures (e.g., composition anomalies, magnetic cloud topology) remain and disclose their origin.

\subsection{CMEs, ICMEs, and space weather}

The compressed sheath plasma behind shocks and the ejecta clouds may both cause substantial deviations of the magnetic field direction from the usual Parker spiral, including strong, out-ofthe-ecliptic components. In either case, a southward pointing IMF ( $B_{\mathrm{z}}$ south) may result, with well-known consequences on the Earth's geomagnetism (Tsurutani et al., 1988; Gonzalez et al., 1999; Huttunen et al., 2002, 2005).

It is important to keep in mind that the sources of magnetic field deflections in the sheath plasma and the ejecta are of fundamentally different origin:

- The field line deflection in the sheath due to draping depends on the orientation of the ejecta relative to the heliospheric current sheet and to the observer sitting, say, near the Earth's bow shock. Thus, the field orientations in the sheath vary dramatically from case to case. In some events a southward component never occurs, while in others it lasts for several hours. The compressed, high-density sheath plasma puts the magnetosphere under additional pressure, and in conjunction with a $B_{\mathrm{z}}$ south episode the resulting geomagnetic storms may become particularly severe.

- The magnetic topology inside ejecta clouds is not yet well understood. It is unclear where the filament lies within an erupting CME and how it is transformed into what becomes the ICME later. Even so, the filament's pre-eruption orientation is often reflected in the ICME configuration. In accordance with the filament's original orientation, the field vector inside the ICME might point to the south at first, then rotate to the east (or west), and finally to the north (SEN and SWN topologies as named by Bothmer and Schwenn, 1998. Due to the reversed magnetic polarity of the Sun in the succeeding activity cycle, the northward swing will then occur first (NES and NWS). Figure 34 shows an example of this particular type. With every other activity cycle, $B_{\mathrm{z}}$ south occurs predominantly at the front or the rear of the clouds, respectively. This applies to filaments with axes close to the ecliptic plane (Bothmer and Schwenn, 1998), as are usually encountered around solar activity minimum. At times of increased solar activity, perpendicular axis orientations are also possible, leading to the corresponding topologies (ESW, ENW, WSE, WNE). Note that half of these latter cloud topologies at high solar activity never have a southward $B_{\mathrm{z}}$ at all. Consequently, those ICMEs are not expected to cause any geomagnetic disturbances. This might explain in part the lowered occurrence rate of geomagnetic storms around maximum activity, as suggested by Mulligan et al. (1998). For the set of magnetic clouds that occurred between 1997 and 2002, Huttunen et al. (2005) found that the majority of magnetic clouds with perpendicular axis orientation occurred in 1997 and 1998, i.e., during the early rising phase of solar activity. Since orientation and helicity of filaments before eruption is often reflected in the topology of the resulting magnetic clouds (Bothmer and Schwenn, 1998; Yurchyshyn et al., 2001), we can use this knowledge for optimizing the prediction of geomagnetic effects (McAllister et al., 2001; Zhao et al., 2001).

The enormous bandwidth of CME properties is of course reflected in the properties of the related ICMEs and their effects. Tsurutani et al. (2004b) analyzed particularly slow magnetic clouds and found them to be surprisingly geo-effective. A good example is the famous event on

Living Reviews in Solar Physics

http://www. livingreviews.org/lrsp-2006-2 


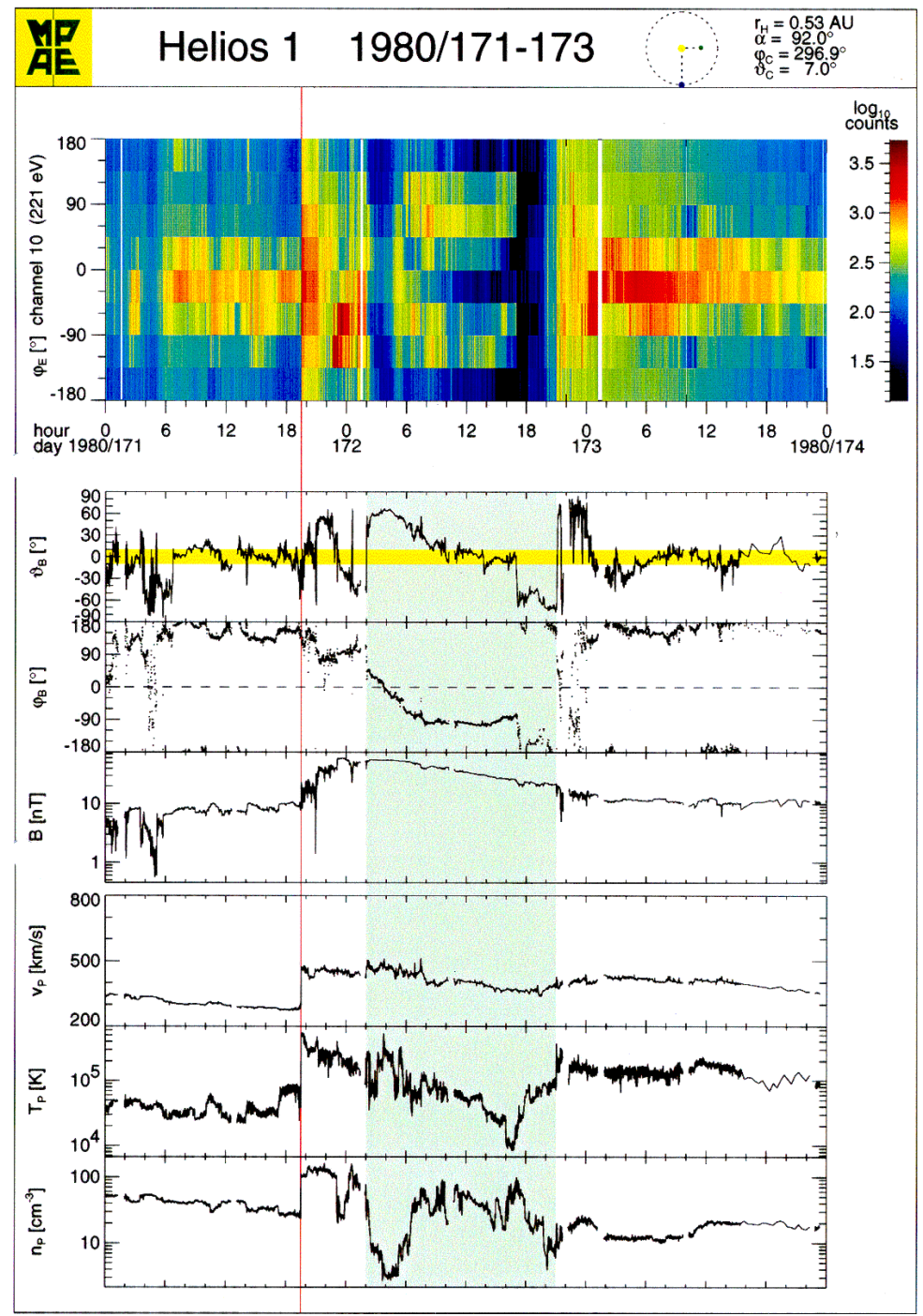

Figure 34: This shock event was observed by the Helios 1 solar probe during 3 days in 1981, at a distance from the Sun of $0.53 \mathrm{AU}$ and at $92^{\circ}$ west of the Earth-Sun line, i.e., right above the Sun's west limb as seen from the Earth. The panels show the solar wind parameters (from bottom to top): proton density, temperature, flow speed, magnetic field magnitude and its azimuth and elevation angles. The upper panel shows the azimuth flow direction of suprathermal electrons (at $221 \mathrm{eV}$ ), the direction away from the Sun being at $180^{\circ}$. The jumps in all parameters and the sudden widening of the electron angular distribution at 19:20 UT on day 171 denotes the arrival of a fast shock wave. The time between 02:00 and 19:00 UT (shaded area) on day 172 denotes the passage of a magnetic cloud, with its characteristic change of the field direction in the sense of a magnetic flux rope, and the simultaneous appearance of oppositely flowing (bi-directional) suprathermal electron streams. Note also the mono-directional electron flow before and after the event series. This one of the cases for which Sheeley Jr et al. (1985), using the SOLWIND coronagraph, could observe a uniquely correlated CME. From Schwenn et al. (2005). 
January 6, 1997: A comparatively slow, unsuspiciously looking, faint partial halo CME caused a problem storm at the Earth 85 hours later, with enormous effects, as described in a series of papers (Zhao and Hoeksema, 1997; Burlaga et al., 1998; Webb et al., 1998). On the other hand, the very fast ICMEs are often found to be responsible for the most intense geomagnetic storms (Srivastava and Venkatakrishnan, 2002; Gonzalez et al., 2004; Yurchyshyn et al., 2004), apparently because they build up extreme ram pressure on the Earths magnetosphere.

The number of CMEs observed at the Sun is about 3 per day at maximum solar activity (St Cyr et al., 2000). Note though that Gopalswamy (2004) found a higher rate since their count includes the extremely faint, narrow and slow CMEs that become only visible due to the very high sensitivity and the enormous dynamic range of the LASCO instruments. At solar maximum, the number of shocks passing an observer located, say, in front of the Earth, is about 0.3 per day (Webb and Howard, 1994). Both rates taken together let us conclude that an in situ observer is hit by only one out of ten ICME shocks released at the Sun. Thus, the average shock shell covers about one tenth of the full solid angle $4 \pi$, i.e., the average shock cone angle as seen from the Suns center amounts to about $100^{\circ}$. This value exceeds significantly the one of the average angular size of the CMEs of about $45^{\circ}$ (Howard et al., 1985; St Cyr et al., 2000). The conclusion is that shock fronts extend much further out in space than their drivers, the ejecta clouds, as had been suggested earlier by Borrini et al. (1982). This explains why an in situ observer finds large numbers of shocks followed by sheath plasma only, with no associated ejecta cloud.

With respect to space weather near the Earth, the crucial question is: How can we forecast the arrival of geoeffective ICMEs? For a CME observed near the Sun, it takes about 1 to 4 days for the associated ICME to reach $1 \mathrm{AU}$, which is a fairly long time for working out a good forecast. However, those CMEs menacing the Earth are usually of the halo type for which the Earth-directed speed component cannot be measured. Several authors tried to use the plane-of-sky speed as listed in the CME catalog as basis for forecasting (e.g. Gopalswamy et al., 2001b; Michałek et al., 2003; Cane and Richardson, 2003). However, the plane-of-sky speed is oriented perpendicularly to the Sun-Earth line and, thus, is not at all representative of the line-of sight speed. Schwenn et al. (2005) found that the lateral expansion speed of a CME which can uniquely be determined for all types of CMEs (for limb CMEs as well as halos) can serve as a proxy value for the inaccessible lineof-sight speed. They associated some 300 candidate CMEs with their ICME signatures observed by in situ spacecraft upstream the Earth. For the 80 uniquely associated event pairs they determined both the CME expansion speed and the travel time to $1 \mathrm{AU}$ as is shown in Figure 35. A fairly good correlation was found. Their empirical formula can be used for forecasting further ICME arrivals, with a $95 \%$ error margin of about 24 hours.

There is no doubt that the scatter in Figure 35 is rather substantial. But the scatter was found to be similarly large even in ideal cases when both the true radial CME speed and the travel times to an in situ observer can precisely be determined (Schwenn et al., 2005). One reason for the scatter is probably the fact that CMEs, on their way through space, travel through very different types of ambient solar wind and have to undergo deceleration or acceleration, depending on the relative speeds. Thus, major deviations from simple kinematic models commonly used for forecasting, may result.

There has been quite some discussion in the literature about the optimum CME acceleration/deceleration model (for details and references see Schwenn et al., 2005). Most authors crosschecked their empirical models with measurements of CME plane-of-sky speeds, which are highly corrupted by projection effects as explained above. Anyway, we should not assign too much meaning to those models, unless we know better the actual propagation environment of the individual CMEs. For the time being, I would rather prefer to join in with Reiner et al. (2003) who, in the spirit of Galileo Galilei, proposed that a number of CMEs be dropped from La Torre di Pisa and their drag force be directly measured.

Of course, the initiation and evolution of CMEs and the resulting propagation of the ejecta

Living Reviews in Solar Physics

http://www. livingreviews.org/lrsp-2006-2 


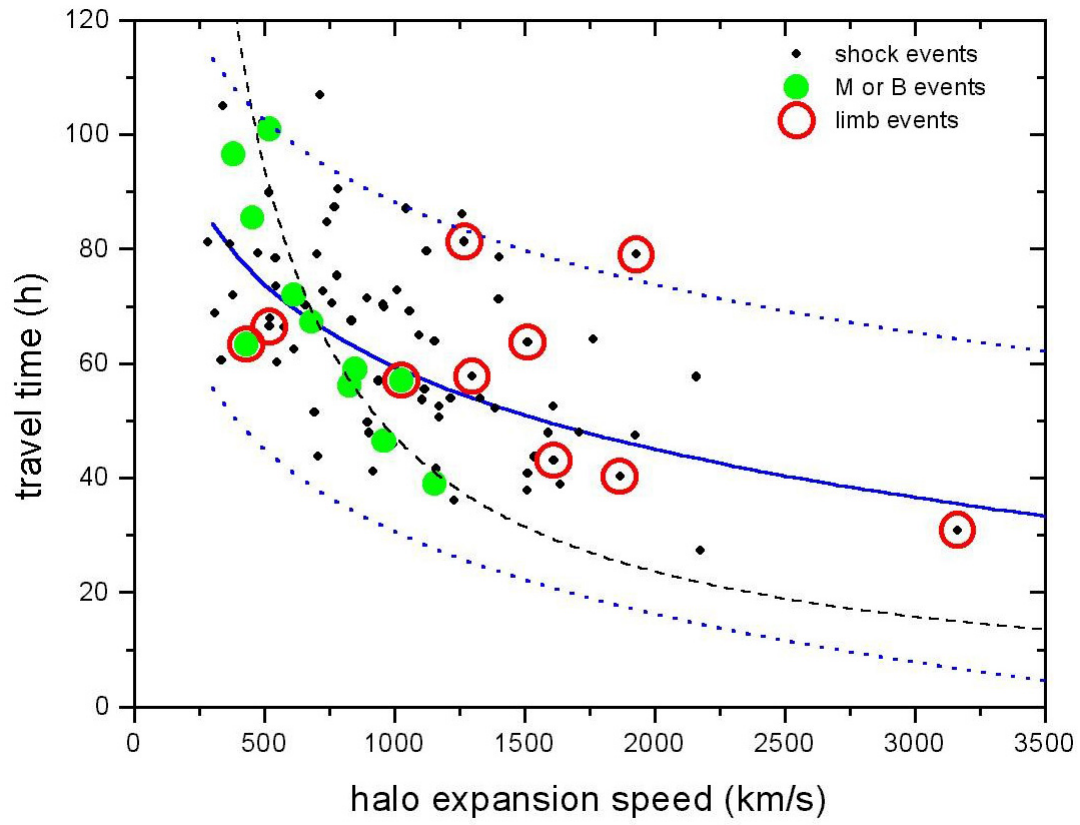

Figure 35: The ICME travel times plotted vs the halo expansion speed $V_{\text {exp }}$ for the 75 usable cases of unique CME-shock correlations. The travel time $T_{\mathrm{tr}}$ is defined by the CME's first appearance in C2 images and the shock arrival at $1 \mathrm{AU}$. The solid line is a least square fit curve to the 80 data points, the fit function being $T_{\mathrm{tr}}=203-20.77 \times \ln \left(V_{\exp }\right)$. The standard deviation from the fit curve is 14 hours. The two dotted lines denote a $95 \%$ certainty margin of two standard deviations. The thin dashed line marks the calculated travel time for a constant radial propagation speed (kinematic approach) inferred from the observed expansion speed according to relation (1). The green dots denote ICMEs without shock signatures, i.e., magnetic clouds (M) and plasma blobs (B). These points were not used for the fit. From Schwenn et al. (2005). 
clouds through the heliosphere have been a key subject for theoreticians and modelers ever since. There is vast literature on various models and numerical codes, some of them being quite controversial. The statement by Riley and Crooker (2004) describes the present status quite well: These models include a rich variety of physics and have been quite successful in reproducing a wide range of observational signatures. However, as the level of sophistication increases, so does the difficulty in interpreting the results. In fact, it is fair to say that at present there is not yet a unified understanding of all processes involved, and we are still searching for the decisive observational facts.

After all, space weather forecasters will have to cope with that 24 hour uncertainty of CME arrival, once they have observed and evaluated a front-side halo CME. The situation is even worse: in $15 \%$ of comparable cases a full or partial halo CME does not cause any ICME signature at Earth at all; every forth partial halo CME and every sixth limb halo CME does not hit the Earth. That would lead to false alarms. Further, every fifth transient shock or ICME or isolated geomagnetic storm is not caused by an identifiable partial or full halo CME on the front side. That would result in missing alarms. We have to admit: our capabilities of forecasting geoeffects based on solar observations is still embarrassingly poor. It seems that the ongoing STEREO mission is about to improve the situation.

For the response of the Earths system to those interplanetary processes the situation is not really better. While the details of their interaction with the magnetosphere are still under study, some empirical relationships continue to be of great use, for example, the famous Burton formula (Burton et al., 1975, see also Lindsay et al., 1999 and Huttunen et al., 2005). It allows to predict the ground-based $D_{\text {st }}$ index solely from a knowledge of the velocity and density of the solar wind and the north-south solar magnetospheric component of the interplanetary magnetic field and seems capable of predicting geomagnetic response during even the largest of storms. The article "Space Weather: Terrestrial Perspective" by Pulkkinen (2007) in Living Reviews in Solar Physics deals with these issues in much more detail. 


\section{Concluding Remarks}

In this review, I had to write at several places statements such as "not yet understood," "waiting for explanation," "our capabilities are embarrassingly poor," etc. This tells us that the field of space weather is still in the state of fundamental research. We are still far away from handling things over to the operators who transform our scientific conclusions into tools for practical application. On the other hand, when comparing our present situation with the one in the 1970s, when the significance of CMEs for solar-terrestrial relations (the term "space weather" was not even coined then) became apparent, I see substantial progress. We can now understand the system to such a degree that we are able to at least identify the basic gaps. That knowledge should allow us to direct future research such that one day the gaps might be closed.

Let me briefly summarize what I consider the key problems:

1. How can we predict flares and CMEs before they actually occur, in terms of event time, location on the Sun and strength? What are the appropriate potential pre-event signatures?

2. How can we predict the fluxes of relativistic SEPs released in context with solar transients?

3. How can we predict the spread of these SEP fluxes through the heliosphere in order to warn in time and protect spaceships on the way to Moon and Mars?

4. How do ICMEs propagate through the ambient interplanetary medium? What are the ingredients needed for an effective real-time computer code that would allow to predict the arrival of an ICME well before it actually arrives?

Of course, each one of these issues can be broken up into many sub-issues, as was indicated at many places in the review.

In the next few years, space weather research will experience another major boost. On the one hand, there are several space missions on the way: SOHO (since 1995, to be continued for several more years), the Advanced Composition Explorer (ACE, since 1997, to be continued for several more years), the WIND spacecraft (since 1994), STEREO A and B, (launched in 2006), Hinode (launched in 2006), the Solar Dynamics Observatory (SDO, launched in 2010), and maybe others in the distant future. NASA's Living With a Star Program (LWS) and the International Living With a Star Program (ILWS) will certainly help to promote research in our field. On the other hand, one must note that any initiative for the Exploration of Moon and Mars depends largely on progress of understanding space weather in that future astronauts must not be exposed to dangerous radiation doses from solar flares.

There is still much fundamental research on the Sun as the driver of space weather waiting to be done. 


\section{References}

Alfvén, H., 1977, "Electric currents in cosmic plasmas", Rev. Geophys. Space Phys., 15, 271-284. [DOI], [ADS] (Cited on pages 13 and 14.)

Alvarez, H., Haddock, F. and Lin, R.P., 1972, "Evidence for Electron Excitation of Type III Radio Burst Emission", Solar Phys., 26, 468. [DOI], [ADS] (Cited on page 36.)

Aschwanden, M.J., 2004, Physics of the Solar Corona: An Introduction, Springer-Praxis Books in Geophysical Sciences, Springer; Praxis, Berlin; New York; Chichester. [Google Books]. Online version (accessed 5 March 2006):

http://www.Imsal.com/ aschwand/eprints/2004_book/ (Cited on page 22.)

Baker, D.N., 2004, "Introduction to Space Weather", in Space Weather: The Physics Behind a Slogan, (Eds.) Scherer, K., Fichtner, H., Heber, B., Mall, U., Papers presented at a spring school organized by the Arbeitsgemeinschaft Extraterrestrische Forschung (AEF), held March 30 -April 4, 2003, vol. 656 of Lecture Notes in Physics, p. 3, Springer, Berlin; New York. [ADS] (Cited on page 5.)

Balmaceda, L., dal Lago, A., Stenborg, G., Francile, C., Gonzalez, W.D. and Schwenn, R., 2003, "Continuous tracking of CMEs using MICA, and LASCO C2 and C3 coronagraphs", Adv. Space Res., 32, 2625-2630. [ADS] (Cited on page 47.)

Balogh, A., Marsden, R.G. and Smith, E.J. (Eds.), 2001, The Heliosphere Near Solar Minimum: The Ulysses Perspective, Springer-Praxis Books in Astrophysics and Astronomy, Springer, London; New York. [ADS] (Cited on page 10.)

Bame, S.J., Asbridge, J.R., Feldman, W.C. and Gosling, J.T., 1977, "Evidence for a structure-free state at high solar wind speeds", J. Geophys. Res., 82, 1487-1492. [DOI], [ADS] (Cited on page 11.)

Bame, S.J., Asbridge, J.R., Feldman, W.C., Fenimore, E.E. and Gosling, J.T., 1979, "Solar wind heavy ions from flare-heated coronal plasma", Solar Phys., 62, 179-201. [DOI], [ADS] (Cited on page 47.)

Bartels, J., 1932, "Terrestrial-magnetic activity and its relations to solar phenomena", Terr. Magn. Atmos. Electr., 37, 1-52. [DOI] (Cited on pages 18 and 21.)

Baumback, M.M., Kurth, W.S. and Gurnett, D.A., 1976, "Direction-finding measurements of type III radio bursts out of the ecliptic plane", Solar Phys., 48, 361-380. [ADS] (Cited on page 27.)

Belcher, J.W. and Davis Jr, L., 1971, "Large amplitude Alfvén waves in the interplanetary medium", J. Geophys. Res., 76, 3534-3563. [DOI] (Cited on page 17.)

Benka, S.G. and Holman, G.D., 1992, "A thermal/nonthermal model for solar microwave bursts", Astrophys. J., 391, 854-864. [DOI], [ADS] (Cited on page 23.)

Benz, A.O., 2008, "Flare Observations", Living Rev. Solar Phys., 5, lrsp-2008-1. URL (accessed 27 May 2010):

http://www.livingreviews.org/lrsp-2008-1 (Cited on page 21.)

Bieber, J.W., Evenson, P., Dröge, W., Pyle, R., Ruffolo, D., Rujiwarodom, M., Tooprakai, P. and Khumlumlert, T., 2004, "Spaceship Earth Observations of the Easter 2001 Solar Particle Event", Astrophys. J. Lett., 601, L103-L106. [DOI], [ADS] (Cited on page 37.) 
Bieber, J.W., Clem, J., Evenson, P., Pyle, R., Ruffolo, D. and Sáiz, A., 2005, "Relativistic solar neutrons and protons on 28 October 2003", Geophys. Res. Lett., 32, 3. [DOI], [ADS] (Cited on page 38.)

Borrini, G., Gosling, J.T., Bame, S.J. and Feldman, W.C., 1982, "An analysis of shock wave disturbances observed at 1 AU from 1971 through 1978", J. Geophys. Res., 87, 4365-4373. [DOI], [ADS] (Cited on page 50.)

Bothmer, V. and Schwenn, R., 1998, "The structure and origin of magnetic clouds in the solar wind", Ann. Geophys., 16, 1-24. [DOI], [ADS] (Cited on pages 47 and 48.)

Bravo, S., Blanco-Cano, X. and López, C., 1999, "Characteristics of interplanetary magnetic clouds in relation to their solar association", J. Geophys. Res., 104, 581-592. [DOI], [ADS] (Cited on page 47.)

Brodrick, D., Tingay, S.J. and Wieringa, M.H., 2005, "X-ray magnitude of the 4 November 2003 solar flare inferred from the ionospheric attenuation of the galactic radio background", J. Geophys. Res., 110, A09S36. [DOI], [ADS] (Cited on page 7.)

Brueckner, G.E., 1974, "The Behaviour of the Outer Solar Corona $\left(3 R_{\odot}\right.$ to $10 R_{\odot}$ during a Large Solar Flare Observed from OSO-7 in White Light", in Coronal Disturbances, (Ed.) Newkirk Jr, G., IAU Symposium no. 57, held at Surfers Paradise, Queensland, Australia, 7-11 September, 1973, pp. 333-334, Reidel, Dordrecht; Boston. [ADS] (Cited on page 39.)

Bruno, R. and Carbone, V., 2005, "The Solar Wind as a Turbulence Laboratory", Living Rev. Solar Phys., 2, lrsp-2005-4. URL (accessed 5 March 2006): http://www.livingreviews.org/lrsp-2005-4 (Cited on page 10.)

Bruno, R., Villante, U., Bavassano, B., Schwenn, R. and Mariani, F., 1986, "In-situ observations of the latitudinal gradients of the solar wind parameters during 1976 and 1977", Solar Phys., 104, 431-445. [DOI], [ADS] (Cited on page 12.)

Burkepile, J.T., Hundhausen, A.J., Stanger, A.L., St Cyr, O.C. and Seiden, J.A., 2004, "Role of projection effects on solar coronal mass ejection properties: 1. A study of CMEs associated with limb activity", J. Geophys. Res., 109, 3103. [DOI], [ADS] (Cited on pages 39 and 44.)

Burlaga, L.F., 1983, "Corotating pressure waves without fast streams in the solar wind", J. Geophys. Res., 88, 6085-6094. [DOI], [ADS] (Cited on page 17.)

Burlaga, L.F., 1991, "Magnetic clouds", in Physics of the Inner Heliosphere, Vol. II: Particles, Waves and Turbulence, (Eds.) Schwenn, R., Marsch, E., vol. 20 of Physics and Chemistry in Space, pp. 1-22, Springer, Berlin; New York (Cited on page 47.)

Burlaga, L.F., 2001, "Terminology for Ejecta in the Solar Wind", Eos Trans. AGU, 82, 433. [DOI]. Online version (accessed 16 June 2010):

http://www .exp-astro.phys.ethz.ch/astro1/Users/benz/papers/divII/WG_

Nomenclature.htm (Cited on page 47.)

Burlaga, L.F., Sittler, E., Mariani, F. and Schwenn, R., 1981, "Magnetic loop behind an interplanetary shock: Voyager, Helios, and IMP 8 observations", J. Geophys. Res., 86, 6673-6684. [DOI], [ADS] (Cited on page 47.)

Burlaga, L.F., Fitzenreiter, R.J., Lepping, R.P., Ogilvie, K.W., Szabo, A., Lazarus, A.J., Steinberg, J.T., Gloeckler, G., Howard, R.A., Michels, D.J., Farrugia, C.J., Lin, R.P. and Larson, D.E., 1998, "A magnetic cloud containing prominence material: January 1997", J. Geophys. Res., 103 (A1), 277-285. [DOI], [ADS] (Cited on page 50.) 
Burton, R.K., McPherron, R.L. and Russell, C.T., 1975, "An empirical relationship between interplanetary conditions and Dst", J. Geophys. Res., 80, 4204-4214. [DOI], [ADS] (Cited on page 52.)

Cane, H.V., 1985, "The evolution of interplanetary shocks", J. Geophys. Res., 90, 191-197. [DOI], [ADS] (Cited on page 29.)

Cane, H.V., 2003, "Near-Relativistic Solar Electrons and Type III Radio Bursts", Astrophys. J., 598, 1403-1408. [DOI], [ADS] (Cited on page 37.)

Cane, H.V. and Erickson, W.C., 2005, "Solar Type II Radio Bursts and IP Type II Events", Astrophys. J., 623, 1180-1194. [DOI], [ADS] (Cited on pages 28, 29, and 30.)

Cane, H.V. and Reames, D.V., 1988, "Some statistics of solar radio bursts of spectral types II and IV", Astrophys. J., 325, 901-904. [DOI], [ADS] (Cited on page 29.)

Cane, H.V. and Richardson, I.G., 2003, "Interplanetary coronal mass ejections in the near-Earth solar wind during 1996-2002", J. Geophys. Res., 108(A4), 1156. [DOI], [ADS] (Cited on page 50.)

Cane, H.V., Richardson, I.G., von Rosenvinge, T.T. and Wibberenz, G., 1994, "Cosmic ray decreases and shock structure: A multispacecraft study", J. Geophys. Res., 99, 21,429-21,441. [DOI], [ADS] (Cited on page 47.)

Chen, J. and Krall, J., 2003, "Acceleration of coronal mass ejections", J. Geophys. Res., 108(A11), 1410. [DOI], [ADS] (Cited on page 44.)

Chernosky, E.J., 1966, "Double Sunspot-Cycle Variation in Terrestrial Magnetic Activity 18841963", J. Geophys. Res., 71, 965. [ADS] (Cited on page 17.)

Chupp, E.L., Forrest, D.J., Ryan, J.M., Heslin, J., Reppin, C., Pinkau, K., Kanbach, G., Rieger, E. and Share, G.H., 1982, "A direct observation of solar neutrons following the 0118 UT flare on 1980 June 21", Astrophys. J. Lett., 263, L95-L99. [DOI], [ADS] (Cited on page 37.)

Chupp, E.L., Debrunner, H., Flueckiger, E., Forrest, D.J., Golliez, F., Kanbach, G., Vestrand, W.T., Cooper, J. and Share, G., 1987, "Solar neutron emissivity during the large flare on 1982 June 3", Astrophys. J., 318, 913-925. [DOI], [ADS] (Cited on page 37.)

Cliver, E.W., 1995, "Solar flare nomenclature", Solar Phys., 157, 285-293. [DOI], [ADS] (Cited on page 21.)

Cliver, E.W., 1999, "Comment on "Origin of coronal and interplanetary shocks: A new look with Wind spacecraft data" by N. Gopalswamy et al.", J. Geophys. Res., 104, 4743-4748. [DOI], [ADS] (Cited on page 29.)

Cliver, E.W. and Cane, H.V., 2002, "Gradual and Impulsive Solar Energetic Particle Events", Eos Trans. AGU, 83, 61-68. [DOI]. Online version (accessed 16 June 2010): http://www.exp-astro.phys.ethz.ch/astro1/Users/benz/papers/benz/divII/WG_ Nomenclature.htm (Cited on page 35.)

Cliver, E.W. and Svalgaard, L., 2004, "The 1859 Solar Terrestrial Disturbance And the Current Limits of Extreme Space Weather Activity", Solar Phys., 224, 407-422. [DOI], [ADS] (Cited on pages 7 and 21.)

Cliver, E.W., Webb, D.F. and Howard, R.A., 1999, "On the origin of solar metric type II bursts", Solar Phys., 187, 89-114. [DOI], [ADS] (Cited on page 29.) 
Cliver, E.W., Kahler, S.W. and Reames, D.V., 2004, "Coronal Shocks and Solar Energetic Proton Events", Astrophys. J., 605, 902-910. [DOI], [ADS] (Cited on page 29.)

Cremades, H. and Bothmer, V., 2004, "On the three-dimensional configuration of coronal mass ejections", Astron. Astrophys., 422, 307-322. [DOI], [ADS] (Cited on pages 39 and 44.)

Crooker, N., Joselyn, J.A. and Feynman, J. (Eds.), 1997, Coronal Mass Ejections, vol. 99 of Geophysical Monograph, American Geophysical Union, Washington, DC (Cited on page 39.)

Crooker, N.U., 2000, "Solar and heliospheric geoeffective disturbances", J. Atmos. Sol.-Terr. Phys., 62, 1071-1085. [DOI], [ADS] (Cited on page 5.)

Daglis, I., Baker, D., Kappenman, J., Panasyuk, M. and Daly, E., 2004, "Effects of space weather on technology infrastructure", Space Weather, 2, S02004. [DOI], [ADS] (Cited on page 5.)

De Jager, C., 1965, "Solar X radiation", Ann. Astrophys., 28, 125-131. [ADS] (Cited on page 23.)

Evenson, P., Meyer, P., Yanagita, S. and Forrest, D.J., 1984, "Electron-rich particle events and the production of gamma-rays by solar flares", Astrophys. J., 283, 439-449. [DOI], [ADS] (Cited on page 36.)

Feldman, W.C., Asbridge, J.R., Bame, S.J. and Gosling, J.T., 1976, "High-speed solar wind flow parameters at 1 AU", J. Geophys. Res., 81, 5054-5060. [DOI], [ADS] (Cited on page 11.)

Fisk, L.A., 1996, "Motion of the footpoints of heliospheric magnetic field lines at the Sun: Implications for recurrent energetic particle events at high heliographic latitudes", J. Geophys. Res., 101, 15 547-15 554. [DOI], [ADS] (Cited on page 17.)

Garcia, H.A., 2004, "Forecasting methods for occurrence and magnitude of proton storms with solar hard X rays", Space Weather, 2, S06003. [ADS] (Cited on page 23.)

Geiss, J., Gloeckler, G. and von Steiger, R., 1995, "Origin of the Solar Wind From Composition Data", Space Sci. Rev., 72, 49. [DOI], [ADS] (Cited on page 15.)

Goldstein, R., Neugebauer, M. and Clay, D., 1998, "A statistical study of coronal mass ejection plasma flows", J. Geophys. Res., 103, 4761. [DOI], [ADS] (Cited on page 47.)

Gonzalez, W.D., Tsurutani, B.T. and Clúa de Gonzalez, A.L., 1999, "Interplanetary origin of geomagnetic storms", Space Sci. Rev., 88, 529-562. [DOI], [ADS] (Cited on page 48.)

Gonzalez, W.D., dal Lago, A., Clúa de Gonzalez, A.L., Vieira, L.E.A. and Tsurutani, B.T., 2004, "Prediction of peak-Dst from halo CME/magnetic cloud-speed observations", J. Atmos. Sol.Terr. Phys., 66, 161-165. [DOI], [ADS] (Cited on page 50.)

Gopalswamy, N., 2004, "A global picture of CMEs in the inner heliosphere", in The Sun and the Heliosphere as an Integrated System, (Eds.) Poletto, G., Suess, S.T., vol. 317 of Astrophysics and Space Science Library, pp. 201-251, Kluwer, Dordrecht. [Google Books]. Online version (accessed 29 May 2006):

http://cdaw.gsfc.nasa.gov/publications/\#gopal2004.assl_cme (Cited on pages 39, 40, and 50.)

Gopalswamy, N., Kaiser, M.L., Lepping, R.P., Kahler, S.W., Ogilvie, K.W., Berdichevsky, D., Kondo, T., Isobe, T. and Akioka, M., 1998, "Origin of coronal and interplanetary shocks: A new look with WIND spacecraft data", J. Geophys. Res., 103, 307-316. [DOI], [ADS] (Cited on page 29.) 
Gopalswamy, N., Lara, A., Kaiser, M.L. and Bougeret, J.-L., 2001a, "Near-Sun and near-Earth manifestations of solar eruptions", J. Geophys. Res., 106(A11), 25,261-25,278. [DOI], [ADS] (Cited on page 37.)

Gopalswamy, N., Lara, A., Yashiro, S., Kaiser, M.L. and Howard, R.A., 2001b, "Predicting the 1-AU arrival times of coronal mass ejections", J. Geophys. Res., 106(A12), 29,207-29,218. [DOI], [ADS] (Cited on page 50.)

Gopalswamy, N., Aguilar-Rodriguez, E., Yashiro, S., Nunes, S., Kaiser, M.L. and Howard, R.A., 2005a, "Type II radio bursts and energetic solar eruptions", J. Geophys. Res., 110, A12S07. [DOI], [ADS] (Cited on page 30.)

Gopalswamy, N., Yashiro, S., Liu, Y., Michalek, G., Vourlidas, A., Kaiser, M.L. and Howard, R.A., 2005b, "Coronal mass ejections and other extreme characteristics of the 2003 October-November solar eruptions", J. Geophys. Res., 110, A09S15. [DOI], [ADS] (Cited on page 7.)

Gosling, J.T., 1990, "Coronal mass ejections and magnetic flux ropes in interplanetary space", in Physics of Magnetic Flux Ropes, (Eds.) Russell, C.T., Priest, E.R., Lee, L.C., Based on papers presented at the AGU Chapman Conference, held in Hamilton, Bermuda on March $27-$ 31, 1989, vol. 58 of Geophysical Monograph Series, pp. 343-364, American Geophysical Union, Washington, DC (Cited on page 47.)

Gosling, J.T., 1993, "The solar flare myth", J. Geophys. Res., 98, 18937-18950. [DOI], [ADS] (Cited on page 31.)

Gosling, J.T. and Pizzo, V.J., 1999, "Formation and Evolution of Corotating Interaction Regions and their Three Dimensional Structure", Space Sci. Rev., 89, 21-52. [DOI], [ADS] (Cited on page 17.)

Gosling, J.T., Pizzo, V. and Bame, S.J., 1973, "Anomalously low proton temperatures in the solar wind following interplanetary shock waves-Evidence for magnetic bottles?", J. Geophys. Res., 78, 2001-2009. [DOI] (Cited on page 47.)

Gosling, J.T., Hildner, E., MacQueen, R.M., Munro, R.H., Poland, A.I. and Ross, C.L., 1974, "Mass ejections from the sun - A view from SKYLAB", J. Geophys. Res., 79, 4581-4587. [DOI], [ADS] (Cited on page 39.)

Gosling, J.T., Hundhausen, A.J. and Bame, S.J., 1976, "Solar wind stream evolution at large heliocentric distances - Experimental demonstration and the test of a model", J. Geophys. Res., 81, 2111-2122. [DOI], [ADS] (Cited on page 15.)

Gosling, J.T., Baker, D.N., Bame, S.J., Feldman, W.C., Zwickl, R.D. and Smith, E.J., 1987, "Bidirectional solar wind electron heat flux events", J. Geophys. Res., 92, 8519-8535. [DOI], [ADS] (Cited on page 47.)

Gurnett, D.A., Baumback, M.M. and Rosenbauer, H., 1978, "Stereoscopic Direction Finding Analysis of a Type III Solar Radio Burst: Evidence for Emission at $2 f_{p}^{-}$", J. Geophys. Res., 83, 616-622. [DOI]. Online version (accessed 5 March 2006):

http://www-pw.physics.uiowa.edu/ dag/publications.html (Cited on page 27.)

Gurnett, D.A., Anderson, R.R. and Tokar, R.L., 1980, "Plasma oscillations and the emissivity of type III radio bursts", in Radio Physics of the Sun, (Eds.) Kundu, M.R., Gergely, T.E., IAU symposium no. 86, held in College Park, Md. U.S.A., August 7-10, 1979, pp. 369-379, D. Reidel, Dordrecht; Boston. [ADS] (Cited on page 25.)

Living Reviews in Solar Physics

http://www.livingreviews.org/lrsp-2006-2 
Haggerty, D.K. and Roelof, E.C., 2002, "Impulsive Near-relativistic Solar Electron Events: Delayed Injection with Respect to Solar Electromagnetic Emission", Astrophys. J., 579, 841-853. [DOI], [ADS] (Cited on page 37.)

Haigh, J.D., 2007, "The Sun and the Earth's Climate", Living Rev. Solar Phys., 4, lrsp-2007-2. URL (accessed 27 May 2010):

http://www.livingreviews.org/lrsp-2007-2 (Cited on page 5.)

Harrison, R.A., 2003, "Soho observations relating to the association between flares and coronal mass ejections", Adv. Space Res., 32, 2425-2437. [ADS] (Cited on page 31.)

Henke, T., Woch, J., Mall, U., Livi, S., Wilken, B., Schwenn, R., Gloeckler, G., von Steiger, R., Forsyth, R.J. and Balogh, A., 1998, "Differences in the $\mathrm{O}^{7+} / \mathrm{O}^{6+}$ ratio of magnetic cloud and non-cloud Coronal Mass Ejections", Geophys. Res. Lett., 25, 3465-3468. [DOI], [ADS] (Cited on page 47.)

Hildner, E., 1977, "Mass ejections from the solar corona into interplanetary space", in Study of Travelling Interplanetary Phenomena 197r, (Eds.) Shea, M.A., Smart, D.F., Wu, S.T., Proceedings of the L.D. de Feiter Memorial Symposium held in Tel Aviv, Israel, June 7-10, 1977, vol. 71 of Astrophysics and Space Science Library, pp. 3-20, Reidel, Dordrecht; Boston. [ADS] (Cited on page 39.)

Hirshberg, J., Asbridge, J.R. and Robbins, D.E., 1971, "The Helium-Enriched Interplanetary Plasma from the Proton Flares of August/September, 1966", Solar Phys., 18, 313. [DOI], [ADS] (Cited on page 47.)

Holman, G.D., 2003, "The Effects of Low- and High-Energy Cutoffs on Solar Flare Microwave and Hard X-Ray Spectra", Astrophys. J., 586, 606-616. [DOI], [ADS] (Cited on page 23.)

Howard, R.A., Michels, D.J., Sheeley Jr, N.R. and Koomen, M.J., 1982, "The observation of a coronal transient directed at earth", Astrophys. J. Lett., 263, L101-L104. [DOI], [ADS] (Cited on pages 5 and 39.)

Howard, R.A., Sheeley Jr, N.R., Michels, D.J. and Koomen, M.J., 1985, "Coronal mass ejections 1979-1981", J. Geophys. Res., 90, 8173-8191. [DOI], [ADS] (Cited on pages 39, 40, 44, and 50.)

Hudson, H.S., Khan, J.I., Lemen, J.R., Nitta, N.V. and Uchida, Y., 2003, "Soft X-ray observation of a large-scale coronal wave and its exciter", Solar Phys., 212, 121-149. [ADS] (Cited on page 44.)

Hundhausen, A.J., 1972, Coronal Expansion and the Solar Wind, vol. 5 of Physics and Chemistry in Space, Springer, Berlin; New York (Cited on pages 10 and 13.)

Hundhausen, A.J., 1987, "The Origin and Propagation of Coronal Mass Ejections (R)", in Solar Wind Six, (Eds.) Pizzo, V.J., Holzer, T., Sime, D.G., Proceedings of the Sixth International Solar Wind Conference held 23-28 August, 1987 at YMCA of the Rockies, Estes Park, Colorado, NCAR Technical Notes, p. 181, Natl. Cent. for Atmos. Res., Boulder. [ADS] (Cited on pages 39 and 40.)

Hundhausen, A.J., 1997, "Coronal mass ejections", in Cosmic Winds and the Heliosphere, (Eds.) Jokipii, J.R., Sonett, C.P., Giampapa, M.S., pp. 259-296, University of Arizona Press, Tucson (Cited on page 39.)

Hundhausen, A.J., Sawyer, C.B., House, L., Illing, R.M.E. and Wagner, W.J., 1984, "Coronal mass ejections observed during the solar maximum mission - Latitude distribution and rate of occurrence", J. Geophys. Res., 89, 2639-2646. [DOI], [ADS] (Cited on pages 39 and 40.) 
Hurford, G.J., Schwartz, R.A., Krucker, S., Lin, R.P., Smith, D.M. and Vilmer, N., 2003, "First Gamma-Ray Images of a Solar Flare", in International Cosmic Ray Conference, (Eds.) Kajita, T., Asaoka, Y., Kawachi, A., Matsubara, Y., Sasaki, M., Proceedings of the 28th International Cosmic Ray Conference, July 31-August 7, 2003, Trukuba, Japan, pp. 3203-3206, Universal Academy Press, Tokyo, Japan. [ADS] (Cited on page 23.)

Huttunen, K.E.J., Koskinen, H.E.J., Schwenn, R. and Dal Lago, A., 2002, "Causes of major magnetic storms near the latest solar maximum", in Solar Variability: From Core to Outer Frontiers, (Ed.) Wilson, A., Proceedings of the 10th European Solar Physics Meeting, Prague, Czech Republic, 9-14 September 2002, vol. SP-506 of ESA Conference Proceedings, pp. 137-140, ESA, Noordwijk (Cited on page 48.)

Huttunen, K.E.J., Schwenn, R., Bothmer, V. and Koskinen, H.E.J., 2005, "Properties and geoeffectiveness of magnetic clouds in the rising, maximum and early declining phases of solar cycle 23", Ann. Geophys., 23, 625-641. [ADS] (Cited on pages 48 and 52.)

Innes, D.E., Curdt, W., Schwenn, R., Solanki, S., Stenborg, G. and McKenzie, D.E., 2001, "Large Doppler Shifts in X-Ray Plasma: An Explosive Start to Coronal Mass Ejection", Astrophys. J. Lett., 549, L249-L252. [DOI], [ADS] (Cited on page 47.)

Jackman, C.H., DeLand, M.T., Labow, G.J., Fleming, E.L., Weisenstein, D.K., Ko, M.K.W., Sinnhuber, M. and Russell, J.M., 2005, "Neutral atmospheric influences of the solar proton events in October-November 2003", J. Geophys. Res., 110, 9. [DOI], [ADS] (Cited on page 38.)

Kahler, S.W., 1992, "Solar flares and coronal mass ejections", Annu. Rev. Astron. Astrophys., 30, 113-141. [DOI], [ADS] (Cited on pages 24, 30, and 39.)

Kahler, S.W., 2001a, "Origin and properties of solar energetic particles in space", in Space Weather, (Ed.) Song, P. et al., vol. 125 of Geophysical Monograph, pp. 109-122, American Geophysical Union, Washington, DC (Cited on page 32.)

Kahler, S.W., 2001b, "The correlation between solar energetic particle peak intensities and speeds of coronal mass ejections: Effects of ambient particle intensities and energy spectra", J. Geophys. Res., 106, 20,947-20,956. [DOI], [ADS] (Cited on page 34.)

Kahler, S.W., Sheeley Jr, N.R., Howard, R.A., Michels, D.J., Koomen, M.J., McGuire, R.E., von Rosenvinge, T.T. and Reames, D.V., 1984, "Associations between coronal mass ejections and solar energetic proton events", J. Geophys. Res., 89, 9683-9693. [DOI], [ADS] (Cited on page 34.)

Kanbach, G., Bertsch, D.L., Fitchel, C.E., Hartman, R.C., Hunter, S.D., Kniffen, D.A., Kwok, P.W., Lin, Y.C., Mattox, J.R. and Mayer-Hasslewander, H.A., 1993, "Detection of a longduration solar gamma-ray flare on June 11, 1991 with EGRET on COMPTON-GRO", Astron. Astrophys. Suppl., 97, 349-353. [ADS] (Cited on page 24.)

Kaufmann, P., Giménez de Castro, C.G., Makhmutov, V.S., Raulin, J.-P., Schwenn, R., Levato, H. and Rovira, M., 2003, "Launch of solar coronal mass ejections and submillimeter pulse bursts", J. Geophys. Res., 108(A7), 1280. [DOI], [ADS] (Cited on pages 23 and 44.)

Kaufmann, P., Raulin, J.-P., de Castro, C.G.G., Levato, H., Gary, D.E., Costa, J.E.R., Marun, A., Pereyra, P., Silva, A.V.R. and Correia, E., 2004, "A New Solar Burst Spectral Component Emitting Only in the Terahertz Range", Astrophys. J. Lett., 603, L121-L124. [DOI], [ADS] (Cited on page 23.)

Kellogg, P.J., 1980, "Fundamental emission in three type III solar bursts", Astrophys. J., 236, 696-700. [DOI], [ADS] (Cited on pages 25 and 26.)

Living Reviews in Solar Physics

http: //www. livingreviews . org/lrsp-2006-2 
Klassen, A., Bothmer, V., Mann, G., Reiner, M.J., Krucker, S., Vourlidas, A. and Kunow, H., 2002, "Solar energetic electron events and coronal shocks", Astron. Astrophys., 385, 1078-1088. [DOI], [ADS] (Cited on page 37.)

Klassen, A., Krucker, S., Kunow, H., Müller-Mellin, R., Wimmer-Schweingruber, R.F., Mann, G. and Posner, A., 2005, "Solar energetic electrons related to the 28 October 2003 flare", J. Geophys. Res., 110, 9. [DOI], [ADS] (Cited on page 37.)

Klein, K.-L., 2003, "Introduction", in Energy Conversion and Particle Acceleration in the Solar Corona, (Ed.) Klein, K.-L., CESRA Workshop and Euroconference, Schloss Ringberg, 2001, vol. 612 of Lecture Notes in Physics, pp. 1-6, Springer, Berlin; New York. [ADS] (Cited on page 38.)

Klein, K.-L., Trottet, G., Lantos, P. and Delaboudinière, J.-P., 2001, "Coronal electron acceleration and relativistic proton production during the 14 July 2000 flare and CME", Astron. Astrophys., 373, 1073-1082. [ADS] (Cited on page 37.)

Kliem, B., MacKinnon, A. and Trottet, G., 2003, "Recent Progress in Understanding Energy Conversion and Particle Acceleration in the Solar Corona", in Energy Conversion and Particle Acceleration in the Solar Corona, (Ed.) Klein, K.-L., CESRA Workshop and Euroconference, Schloss Ringberg, 2001, vol. 612 of Lecture Notes in Physics, pp. 263-293, Springer, Berlin; New York. [ADS] (Cited on page 38.)

Kohl, J.L. and Cranmer, S.R. (Eds.), 1999, Coronal Holes and Solar Wind Acceleration, Proceedings of the SOHO-7 Workshop, held at Asticou Inn in Northeast Harbor, Maine, USA from 28 September-1 October 1998, Kluwer Academic Publishers, Dordrecht; Boston. [Google Books]. Reprinted from Space Sci. Rev., vol. 87, nos. 1-2, 1999 (Cited on page 10.)

Koutchmy, S., Baudin, F., Bocchialini, K., Daniel, J.-Y., Delaboudinière, J.-P., Golub, L., Lamy, P. and Adjabshirizadeh, A., 2004, "The August 11th, 1999 CME", Astron. Astrophys., 420, 709-718. [DOI], [ADS] (Cited on page 11.)

Krieger, A.S., Timothy, A.F. and Roelof, E.C., 1973, "A Coronal Hole and Its Identification as the Source of a High Velocity Solar Wind Stream", Solar Phys., 29, 505-525. [DOI], [ADS] (Cited on page 10.)

Krucker, S. and Lin, R.P., 2002, "Relative Timing and Spectra of Solar Flare Hard X-ray Sources", Solar Phys., 210, 229-243. [ADS] (Cited on page 27.)

Krucker, S., Larson, D.E., Lin, R.P. and Thompson, B.J., 1999, "On the Origin of Impulsive Electron Events Observed at 1 AU", Astrophys. J., 519, 864-875. [DOI], [ADS] (Cited on page 37.)

Kunow, H., Wibberenz, G., Green, G. and Müller-Mellin, R., 1991, "Energetic particles in the inner solar system", in Physics of the Inner Heliosphere, Vol. II: Particles, Waves and Turbulence, (Eds.) Schwenn, R., Marsch, E., vol. 20 of Physics and Chemistry in Space, p. 243, Springer, Berlin; New York (Cited on pages 32 and 36.)

Laitinen, T., Klein, K.-L., Kocharov, L., Torsti, J., Trottet, G., Bothmer, V., Kaiser, M.L., Rank, G. and Reiner, M.J., 2000, "Solar energetic particle event and radio bursts associated with the 1996 July 9 flare and coronal mass ejection", Astron. Astrophys., 360, 729-741. [ADS] (Cited on page 37.)

Lanzerotti, L.J., 2001, "The Solar Sources of Geoeffective Structures", in Space Weather, (Ed.) Song, P. et al., vol. 125 of Geophysical Monograph, pp. 11-22, American Geophysical Union, Washington, DC (Cited on page 5.) 
Lean, J., 1991, "Variations in the Sun's radiative output", Rev. Geophys., 29, 505-535. [DOI], [ADS] (Cited on page 30.)

Lin, R.P., 1985, "Energetic solar electrons in the interplanetary medium", Solar Phys., 100, 537561. [DOI], [ADS] (Cited on page 36.)

Lin, R.P., 2005, "Relationship of solar flare accelerated particles to solar energetic particles (SEPs) observed in the interplanetary medium", Adv. Space Res., 35, 1857-1863. [DOI], [ADS] (Cited on page 27.)

Lin, R.P., Mewaldt, R.A. and van Hollebeke, M.A.I., 1982, "The energy spectrum of 20 keV-20 MeV electrons accelerated in large solar flares", Astrophys. J., 253, 949-962. [DOI], [ADS] (Cited on page 37.$)$

Lin, R.P., Krucker, S., Hurford, G.J., Smith, D.M., Hudson, H.S., Holman, G.D., Schwartz, R.A., Dennis, B.R., Share, G.H., Murphy, R.J., Emslie, A.G., Johns-Krull, C. and Vilmer, N., 2003, "RHESSI Observations of Particle Acceleration and Energy Release in an Intense Solar GammaRay Line Flare", Astrophys. J. Lett., 595, L69-L76. [DOI], [ADS] (Cited on pages 23 and 24.)

Lindsay, G.M., Russell, C.T. and Luhmann, J.G., 1999, "Predictability of Dst index based upon solar wind conditions monitored inside 1 AU", J. Geophys. Res., 104, 10,335-10,344. [DOI], [ADS] (Cited on page 52.)

Low, B.C., 2001, "Coronal mass ejections, magnetic flux ropes, and solar magnetism", J. Geophys. Res., 106, 25,141-25,164. [DOI], [ADS] (Cited on page 39.)

MacQueen, R.M., 1980, "Coronal transients: a summary", Philos. Trans. R. Soc. London, Ser. A, 297, 605-620. [ADS] (Cited on page 39.)

Maia, D.J.F. and Pick, M., 2004, "Revisiting the Origin of Impulsive Electron Events: Coronal Magnetic Restructuring", Astrophys. J., 609, 1082-1097. [DOI], [ADS] (Cited on page 37.)

Mann, G. and Klassen, A., 2002, "Shock accelerated electron beams in the solar corona", in Solar Variability: From Core to Outer Frontiers, (Ed.) Wilson, A., Proceedings of the 10th European Solar Physics Meeting, Prague, Czech Republic, 9-14 September 2002, vol. SP-506 of ESA Conference Proceedings, pp. 245-248, ESA, Noordwijk. [ADS] (Cited on page 28.)

Mann, G. and Klassen, A., 2005, "Electron beams generated by shock waves in the solar corona", Astron. Astrophys., 441, 319-326. [DOI], [ADS] (Cited on pages 28, 29, and 36.)

Mann, G., Klassen, A., Estel, C. and Thompson, B.J., 1999, "Coronal Transient Waves and Coronal Shock Waves", in Plasma Dynamics and Diagnostics in the Solar Transition Region and Corona, (Eds.) Vial, J.-C., Kaldeich-Schü, B., Proceedings of the 8th SOHO Workshop, 22 - 25 June 1999, Paris, France, vol. SP-446 of ESA Conference Proceedings, p. 477, ESA Publications Division, Noordwijk. [ADS] (Cited on page 37.)

Mann, G., Klassen, A., Aurass, H. and Classen, H.-T., 2003, "Formation and development of shock waves in the solar corona and the near-Sun interplanetary space", Astron. Astrophys., 400, 329-336. [DOI], [ADS] (Cited on pages 30 and 37.)

Marsch, E., 1991, "MHD turbulence in the solar wind", in Physics of the Inner Heliosphere, Vol. II: Particles, Waves and Turbulence, (Eds.) Schwenn, R., Marsch, E., vol. 20 of Physics and Chemistry in Space, pp. 159-241, Springer, Berlin; New York (Cited on page 17.)

Living Reviews in Solar Physics

http://www. livingreviews . org/lrsp-2006-2 
Marsch, E., 2006, "Kinetic Physics of the Solar Corona and Solar Wind", Living Rev. Solar Phys., 3, lrsp-2006-1. URL (accessed 5 March 2006): http://www.livingreviews.org/lrsp-2006-1 (Cited on page 10.)

Marsch, E., Axford, W.I. and McKenzie, J.F., 2003, "Solar wind", in Dynamic Sun, (Ed.) Dwivedi, B.N., pp. 374-402, Cambridge University Press, Cambridge. [ADS], [Google Books] (Cited on page 10.)

Martin, S.F., 2003, "Signs of helicity in solar prominences and related features", Adv. Space Res., 32, 1883-1893. [DOI], [ADS] (Cited on page 47.)

Marubashi, K., 1986, "Structure of the interplanetary magnetic clouds and their solar origins", Adv. Space Res., 6, 335-338. [DOI], [ADS] (Cited on page 47.)

McAllister, A.H., Martin, S.F., Crooker, N.U., Lepping, R.P. and Fitzenreiter, R.J., 2001, "A test of real-time prediction of magnetic cloud topology and geomagnetic storm occurrence from solar signatures", J. Geophys. Res., 106, 29,185-29,194. [DOI], [ADS] (Cited on page 48.)

McComas, D.J., Gosling, J.T., Winterhalter, D. and Smith, E.J., 1988, "Interplanetary magnetic field draping about fast coronal mass ejecta in the outer heliosphere", J. Geophys. Res., 93, 2519-2526. [DOI], [ADS] (Cited on page 47.)

McKibben, R.B., Simpson, J.A., Zhang, M., Bame, S. and Balogh, A., 1995, "ULYSSES Out-ofEcliptic Observations of '27-day' Variations in High Energy Cosmic Ray Intensity", Space Sci. Rev., 72, 403. [DOI], [ADS] (Cited on page 17.)

Meadows, A.J., 1970, Early solar physics, Selected Readings in Physics, Pergamon Press, Oxford (Cited on page 21.)

Mewaldt, R.A., Cohen, C.M.S., Labrador, A.W., Leske, R.A., Mason, G.M., Desai, M.I., Looper, M.D., Mazur, J.E., Selesnick, R.S. and Haggerty, D.K., 2005, "Proton, helium, and electron spectra during the large solar particle events of October-November 2003", J. Geophys. Res., 110, A09S18. [DOI], [ADS] (Cited on pages 32 and 33.)

Michałek, G., Gopalswamy, N. and Yashiro, S., 2003, "A New Method for Estimating Widths, Velocities, and Source Location of Halo Coronal Mass Ejections", Astrophys. J., 584, 472-478. [DOI], [ADS] (Cited on page 50.)

Mikić, Z. and Linker, A.J., 1997, "The initiation of coronal mass ejections by magnetic shear", in Coronal Mass Ejections, (Eds.) Crooker, N., Joselyn, J.A., Feynman, J., vol. 99 of Geophysical Monograph, pp. 57-64, American Geophysical Union, Washington, DC (Cited on page 47.)

Miroshnichenko, L.I., Klein, K.-L., Trottet, G., Lantos, P., Vashenyuk, E.V., Balabin, Y.V. and Gvozdevsky, B.B., 2005, "Relativistic nucleon and electron production in the 2003 October 28 solar event", J. Geophys. Res., 110, A09S08. [DOI], [ADS] (Cited on page 38.)

Montgomery, M.D., Asbridge, J.R., Bame, S.J. and Feldman, W.C., 1974, "Solar wind electron temperature depressions following some interplanetary shock waves: Evidence for magnetic merging?", J. Geophys. Res., 79, 3103. [DOI] (Cited on page 47.)

Moon, Y.-J., Choe, G.S., Wang, H., Park, Y.D., Gopalswamy, N., Yang, G. and Yashiro, S., 2002, "A Statistical Study of Two Classes of Coronal Mass Ejections", Astrophys. J., 581, 694-702. [DOI], [ADS] (Cited on page 40.) 
Moore, R.L., Sterling, A.C., Hudson, H.S. and Lemen, J.R., 2001, "Onset of the Magnetic Explosion in Solar Flares and Coronal Mass Ejections", Astrophys. J., 552, 833-848. [DOI], [ADS] (Cited on page 47.)

Moreton, G.E., 1960, "H $\alpha$ Observations of Flare-Initiated Disturbances with Velocities 1000 km/sec", Astron. J., 65, 494-495. [DOI], [ADS] (Cited on page 44.)

Moses, D., Droege, W., Meyer, P. and Evenson, P., 1989, "Characteristics of energetic solar flare electron spectra", Astrophys. J., 346, 523-530. [DOI], [ADS] (Cited on page 37.)

Mulligan, T., Russell, C.T. and Luhmann, J.G., 1998, "Solar cycle evolution of the structure of magnetic clouds in the inner heliosphere", Geophys. Res. Lett., 25, 2959-2962. [DOI], [ADS] (Cited on page 48.)

Nelson, G.J. and Melrose, D.B., 1985, "Type II bursts", in Solar Radiophysics: Studies of Emission from the Sun at Metre Wavelengths, (Eds.) McLean, D.J., Labrum, N.R., pp. 333-359, Cambridge University Press, Cambridge; New York. [ADS] (Cited on page 27.)

Osherovich, V. and Burlaga, L.F., 1997, "Magnetic clouds", in Coronal Mass Ejections, (Eds.) Crooker, N., Joselyn, J.A., Feynman, J., vol. 99 of Geophysical Monograph, pp. 157-168, American Geophysical Union, Washington, DC (Cited on page 47.)

Palmer, I.D., Allum, F.R. and Singer, S., 1978, "Bidirectional anisotropies in solar cosmic ray events - Evidence for magnetic bottles", J. Geophys. Res., 83, 75-90. [DOI], [ADS] (Cited on page 47.)

Parker, E.N., 1958, "Dynamics of the Interplanetary Gas and Magnetic Fields", Astrophys. J., 128, 664-676. [DOI], [ADS] (Cited on page 12.)

Pick, M., Klein, K.-L. and Trottet, G., 1990, "Meter-decimeter and microwave radio observations of solar flares", Astrophys. J. Suppl. Ser., 73, 165-175. [DOI], [ADS] (Cited on page 23.)

Posner, A., Zurbuchen, T.H., Schwadron, N.A., Fisk, L.A., Gloeckler, G., Linker, J.A., Mikić, Z. and Riley, P., 2001, "Nature of the boundary between open and closed magnetic field line regions at the Sun revealed by composition data and numerical models", J. Geophys. Res., 106, 15,869-15,880. [DOI], [ADS] (Cited on page 17.)

Potter, D.W., Lin, R.P. and Anderson, K.A., 1980, "Impulsive 2-10 keV solar electron events not associated with flares", Astrophys. J. Lett., 236, L97-L100. [DOI], [ADS] (Cited on page 36.)

Priest, E.R., 1988, "The initiation of solar coronal mass ejections by magnetic nonequilibrium", Astrophys. J., 328, 848-855. [DOI], [ADS] (Cited on page 47.)

Pulkkinen, T., 2007, "Space Weather: Terrestrial Perspective", Living Rev. Solar Phys., 4, lrsp2007-1. URL (accessed 27 May 2010):

http://www.livingreviews.org/lrsp-2007-1 (Cited on pages 9, 31, and 52.)

Ramaty, R. and Mandzhavidze, N. (Eds.), 2000, High Energy Solar Physics Workshop - Anticipating HESSI, Proceedings of the workshop held at the University of Maryland, October 18-20, 1999, vol. 206 of ASP Conference Series, Astronomical Society of the Pacific, San Francisco. [ADS] (Cited on page 23.)

Ramaty, R., Murphy, R.J., Kozlovsky, B. and Lingenfelter, R.E., 1983, "Implications of highenergy neutron observations from solar flares", Astrophys. J. Lett., 273, L41-L45. [DOI], [ADS] (Cited on page 37.) 
Ramaty, R., Mandzhavidze, N. and Hua, X. (Eds.), 1996, High Energy Solar Physics, Proceedings of the conference held in Greenbelt, Maryland, August 1995, vol. 374 of AIP Conference Proceedings, American Institute of Physics, Woodbury, NY (Cited on page 21.)

Ramaty, R., Mandzhavidze, N. and Murdin, P., 2002, "Solar Flares: Gamma Rays", in Encyclopedia of Astronomy and Astrophysics, (Ed.) Murdin, P., p. 2292, Institute of Physics Publishing, Bristol. [ADS] (Cited on page 24.)

Reames, D.V., 1999, "Particle acceleration at the Sun and in the heliosphere", Space Sci. Rev., 90, 413-491. [DOI], [ADS] (Cited on pages 31, 32, 35, and 36.)

Reames, D.V., 2001, "SEPs: Space Weather Hazard in Interplanetary Space", in Space Weather, (Ed.) Song, P. et al., vol. 125 of Geophysical Monograph, pp. 101-107, American Geophysical Union, Washington, DC (Cited on page 32.)

Reames, D.V., 2002, "Solar Wind: Energetic Particles", in Encyclopedia of Astronomy and Astrophysics, (Ed.) Murdin, P., p. 2312, Institute of Physics Publishing, Bristol. [ADS] (Cited on page 32.)

Reames, D.V., 2004, "Solar energetic particle variations", Adv. Space Res., 34, 381-390. [DOI], [ADS] (Cited on pages 32 and 34.)

Reames, D.V. and Ng, C.K., 2004, "Heavy-Element Abundances in Solar Energetic Particle Events", Astrophys. J., 610, 510-522. [DOI], [ADS] (Cited on page 36.)

Reames, D.V., Barbier, L.M. and Ng, C.K., 1996, "The Spatial Distribution of Particles Accelerated by Coronal Mass Ejection-driven Shocks", Astrophys. J., 466, 473. [DOI], [ADS] (Cited on pages 34 and 35.)

Reeves, G.D., Cayton, T.E., Gary, S.P. and Belian, R.D., 1992, "The great solar energetic particle events of 1989 observed from geosynchronous orbit", J. Geophys. Res., 97, 6219-6226. [DOI], [ADS] (Cited on page 32.)

Reiner, M.J., Fainberg, J. and Stone, R.G., 1995, "Large-Scale Interplanetary Magnetic Field Configuration Revealed by Solar Radio Bursts", Science, 270, 461-464. [DOI], [ADS] (Cited on page 27.)

Reiner, M.J., Kaiser, M.L., Fainberg, J., Bougeret, J.-L. and Stone, R.G., 1998, "On the origin of radio emissions associated with the January 6-11, 1997, CME", Geophys. Res. Lett., 25, 2493-2496. [DOI], [ADS] (Cited on pages 29 and 30.)

Reiner, M.J., Kaiser, M.L. and Bougeret, J.-L., 2001, "Radio signatures of the origin and propagation of coronal mass ejections through the solar corona and interplanetary medium", J. Geophys. Res., 106, 29,989-30,000. [DOI], [ADS] (Cited on pages 29 and 44.)

Reiner, M.J., Kaiser, M.L. and Bougeret, J.-L., 2003, "On the Deceleration of CMEs in the Corona and Interplanetary Medium deduced from Radio and White-Light Observations", in Solar Wind Ten, (Eds.) Velli, M., Bruno, R., Malara, F., Proceedings of the Tenth International Solar Wind Conference, Pisa, Italy, 17 - 21 June 2002, vol. 679 of AIP Conference Proceedings, pp. 152-155, American Institute of Physics, Melville, NY. [ADS] (Cited on page 50.)

Reiner, M.J., Jackson, B.V., Webb, D.F., Mizuno, D.R., Kaiser, M.L. and Bougeret, J.-L., 2005, "Coronal mass ejection kinematics deduced from white light (Solar Mass Ejection Imager) and radio (Wind/WAVES) observations", J. Geophys. Res., 110, 9. [DOI], [ADS] (Cited on page 30.) 
Richardson, I.G. and Cane, H.V., 1995, "Regions of abnormally low proton temperature in the solar wind (1965-1991) and their association with ejecta", J. Geophys. Res., 100, 23,397-23,412. [DOI], [ADS] (Cited on page 47.)

Richardson, R.S., 1944, "Solar Flares Versus Bright Chromospheric Eruptions: A Question of Terminology", Publ. Astron. Soc. Pac., 56, 156. [DOI], [ADS] (Cited on page 21.)

Rieger, E. and Rank, G., 2001, "The Sun as a Gamma-Ray Source", in The Universe in Gamma Rays, (Ed.) Schönfelder, V., pp. 91-126, Springer, Berlin; New York. [ADS], [Google Books] (Cited on page 24.)

Riley, P. and Crooker, N.U., 2004, "Kinematic Treatment of Coronal Mass Ejection Evolution in the Solar Wind", Astrophys. J., 600, 1035-1042. [DOI], [ADS] (Cited on page 52.)

Robbrecht, E., Berghmans, D. and Van der Linden, R.A.M., 2009, "Automated LASCO CME Catalog for Solar Cycle 23: Are CMEs Scale Invariant?", Astrophys. J., 691, 1222-1234. [DOI], [ADS], [arXiv:0810.1252] (Cited on page 40.)

Roberts, J.A., 1959, "Some aspects of type II bursts", in Paris Symposium on Radio Astronomy, (Ed.) Bracewell, R.N., IAU Symposium no. 9 and URSI Symposium no. 1, held from 30 July to 6 August 1958, pp. 194-200, Stanford University Press, Stanford, CA. [ADS] (Cited on page 28.)

Rodriguez, L., Woch, J., Krupp, N., Fränz, M., von Steiger, R., Forsyth, R.J., Reisenfeld, D.B. and Glaßmeier, K.-H., 2004, "A statistical study of oxygen freezing-in temperature and energetic particles inside magnetic clouds observed by Ulysses", J. Geophys. Res., 109, A01108. [DOI], [ADS] (Cited on page 47.)

Rohen, G., von Savigny, C., Sinnhuber, M., Llewellyn, E.J., Kaiser, J.W., Jackman, C.H., Kallenrode, M.-B., Schröter, J., Eichmann, K.-U., Bovensmann, H. and Burrows, J.P., 2005, "Ozone depletion during the solar proton events of October/November 2003 as seen by SCIAMACHY", J. Geophys. Res., 110, A09S39. [DOI], [ADS] (Cited on page 38.)

Rosenbauer, H., Schwenn, R., Marsch, E., Meyer, B., Miggenrieder, H., Montgomery, M.D., Mühlhäuser, K.H., Pilipp, W., Voges, W. and Zink, S.M., 1977, "A survey on initial results of the HELIOS plasma experiment", J. Geophys., 42(6), 561-580. [ADS] (Cited on page 12.)

Rosenberg, R.L. and Coleman Jr, P.J., 1980, "Solar cycle-dependent north-south field configurations observed in solar wind interaction regions", J. Geophys. Res., 85, 3021-3032. [DOI], [ADS] (Cited on page 17.)

Russell, C.T., 1974, "On the heliographic latitude dependence of the interplanetary magnetic field as deduced from the 22-year cycle of geomagnetic activity", Geophys. Res. Lett., 1, 11-12. [DOI]. Online version (accessed 5 March 2006):

http://www-ssc.igpp.ucla.edu/personnel/russell/papers/68/ (Cited on page 17.)

Russell, C.T., 2001, "In Defense of the Term ICME", Eos Trans. AGU, 82, 433-435. [DOI]. Online version (accessed 16 June 2010):

http://www.exp-astro.phys.ethz.ch/astro1/Users/benz/papers/benz/divII/WG_ Nomenclature.htm (Cited on page 47.)

Rust, D.M., 2003, "The helicial flux rope structure of solar filaments", Adv. Space Res., 32, 18951903. [DOI], [ADS] (Cited on page 47.)

Rust, D.M. and Kumar, A., 1996, "Evidence for Helically Kinked Magnetic Flux Ropes in Solar Eruptions", Astrophys. J. Lett., 464, L199-L202. [DOI], [ADS] (Cited on page 47.) 
Scholer, M., Mann, G., Chalov, S., Desai, M.I., Fisk, L.A., Jokipii, J.R., Kallenbach, R., Keppler, E., Kóta, J., Kunow, H., Lee, M.A., Sanderson, T.R. and Simnett, G.M., 1999, "Origin, Injection, and Acceleration of CIR Particles: Theory Report of Working Group 7", Space Sci. Rev., 89, 369-399. [DOI], [ADS] (Cited on page 17.)

Schulz, M., 1973, "Interplanetary Sector Structure and the Heliomagnetic Equator", Astrophys. Space Sci., 24, 371. [DOI], [ADS] (Cited on page 14.)

Schwenn, R., 1981, "Solar wind and its interaction with the magnetosphere - Measured parameters", Adv. Space Res., 1, 3-17. [DOI], [ADS] (Cited on page 18.)

Schwenn, R., 1983, "Direct correlations between coronal transients and interplanetary disturbances", Space Sci. Rev., 34, 85-99. [DOI], [ADS] (Cited on page 30.)

Schwenn, R., 1986, "Relationship of coronal transients to interplanetary shocks 3D aspects", Space Sci. Rev., 44, 139-168. [DOI], [ADS] (Cited on pages 39 and 44.)

Schwenn, R., 1990, "Large-Scale Structure of the Interplanetary Medium", in Physics of the Inner Heliosphere, Vol. I: Large-Scale Phenomena, (Eds.) Schwenn, R., Marsch, E., vol. 20 of Physics and Chemistry in Space, pp. 99-181, Springer, Berlin; New York. [ADS] (Cited on pages 12, 13, 15,16 , and 18.)

Schwenn, R., 1996, "An Essay on Terminology, Myths, and Known Facts: Solar Transient - Flare - CME - Driver Gas - Piston - BDE - Magnetic Cloud - Shock Wave - Geomagnetic Storm", Astrophys. Space Sci., 243, 187-193. [DOI], [ADS] (Cited on page 39.)

Schwenn, R. and Marsch, E. (Eds.), 1990, Physics of the Inner Heliosphere, Vol. I: Large-Scale Phenomena, vol. 20 of Physics and Chemistry in Space, Springer, Berlin; New York. [ADS] (Cited on page 10.)

Schwenn, R. and Marsch, E., 1991, Physics of the Inner Heliosphere, Vol. II: Particles, Waves and Turbulence, vol. 21 of Physics and Chemistry in Space, Springer, Berlin; New York (Cited on page 10.)

Schwenn, R., Montgomery, M.D., Rosenbauer, H., Miggenrieder, H., Mühlhäuser, K.H., Bame, S.J., Feldman, W.C. and Hansen, R.T., 1978, "Direct observation of the latitudinal extent of a high-speed stream in the solar wind", J. Geophys. Res., 83, 1011-1017. [DOI], [ADS] (Cited on page 12.)

Schwenn, R., Rosenbauer, H. and Mühlhäuser, K.-H., 1980, "Singly-ionized helium in the driver gas of an interplanetary shock wave", Geophys. Res. Lett., 7, 201-204. [DOI], [ADS] (Cited on page 47.)

Schwenn, R., Inhester, B., Plunkett, S.P., Epple, A., Podlipnik, B., Bedford, D.K., Eyles, C.J., Simnett, G.M., Tappin, S.J., Bout, M.V., Lamy, P.L., Llebaria, A., Brueckner, G.E., Dere, K.P., Howard, R.A., Koomen, M.J., Korendyke, C.M., Michels, D.J., Moses, J.D., Moulton, N.E., Paswaters, S.E., Socker, D.G., St Cyr, O.C. and Wang, D., 1997, "First View of the Extended Green-Line Emission Corona At Solar Activity Minimum Using the Lasco-C1 Coronagraph on SOHO", Solar Phys., 175, 667-684. [DOI], [ADS] (Cited on pages 14 and 15.)

Schwenn, R., dal Lago, A., Huttunen, E. and Gonzalez, W.D., 2005, "The association of coronal mass ejections with their effects near the Earth", Ann. Geophys., 23, 1033-1059. [ADS] (Cited on pages $7,44,49,50$, and 51.$)$ 
Sheeley Jr, N.R. and Harvey, J.W., 1981, "Coronal holes, solar wind streams, and geomagnetic disturbances during 1978 and 1979", Solar Phys., 70, 237-249. [ADS] (Cited on pages 18 and 20.)

Sheeley Jr, N.R. and Wang, Y.-M., 2002, "Characteristics of Coronal Inflows", Astrophys. J., 579, 874-887. [DOI], [ADS] (Cited on page 44.)

Sheeley Jr, N.R., Howard, R.A., Koomen, M.J., Michels, D.J., Schwenn, R., Mühlhäuser, K.H. and Rosenbauer, H., 1983, "Associations between coronal mass ejections and interplanetary shocks", in Solar Wind Five, (Ed.) Neugebauer, M., Proceedings of a conference held in Woodstock, Vermont, November 1-5, 1982, vol. 2280 of NASA Conference Publication, pp. 693-702, NASA, Washington, DC. [ADS] (Cited on page 30.)

Sheeley Jr, N.R., Howard, R.A., Michels, D.J., Robinson, R.D., Koomen, M.J. and Stewart, R.T., 1984, "Associations between coronal mass ejections and metric type II bursts", Astrophys. J., 279, 839-847. [ADS] (Cited on page 29.)

Sheeley Jr, N.R., Howard, R.A., Michels, D.J., Koomen, M.J., Schwenn, R., Mühlhäuser, K.H. and Rosenbauer, H., 1985, "Coronal mass ejections and interplanetary shocks", J. Geophys. Res., 90, 163-175. [ADS] (Cited on pages 30 and 49.)

Sheeley Jr, N.R., Wang, Y.-M., Hawley, S.H., Brueckner, G.E., Dere, K.P., Howard, R.A., Koomen, M.J., Korendyke, C.M., Michels, D.J., Paswaters, S.E., Socker, D.G., St Cyr, O.C., Wang, D., Lamy, P.L., Llebaria, A., Schwenn, R., Simnett, G.M., Plunkett, S. and Biesecker, D.A., 1997, "Measurements of Flow Speeds in the Corona between 2 and $30 R_{\odot}$ ", Astrophys. J., 484, 472478. [DOI], [ADS] (Cited on page 11.)

Sheeley Jr, N.R., Walters, J.H., Wang, Y.-M. and Howard, R.A., 1999, "Continuous tracking of coronal outflows: Two kinds of coronal mass ejections", J. Geophys. Res., 104, 24,739-24,768. [ADS] (Cited on page 40.)

Simnett, G.M., 1974, "Relativistic Electron Events in Interplanetary Space", Space Sci. Rev., 16, 257. [DOI], [ADS] (Cited on page 37.)

Simnett, G.M., 2003, "Energetic Particles and Coronal Mass Ejections: a Case Study From ace and Ulysses", Solar Phys., 213, 387-412. [DOI], [ADS] (Cited on page 36.)

Singer, H.J., Heckman, G.R. and Hirman, J.W., 2001, "Space weather forecasting: a grand challenge", in Space Weather, (Ed.) Song, P. et al., vol. 125 of Geophysical Monograph, pp. 23-29, American Geophysical Union, Washington, DC (Cited on page 7.)

Siscoe, G., 2000, "The space-weather enterprise: past, present, and future", J. Atmos. Sol.-Terr. Phys., 62, 1223-1232. [DOI], [ADS] (Cited on page 5.)

Siscoe, G.L. and Finley, L.T., 1969, "Meridional (North-South) Motions of the Solar Wind", Solar Phys., 9, 452. [ADS] (Cited on page 17.)

Snyder, C.W., Neugebauer, M. and Rao, U.R., 1963, "The Solar Wind Velocity and Its Correlation with Cosmic-Ray Variations and with Solar and Geomagnetic Activity", J. Geophys. Res., 68, 6361. [ADS] (Cited on page 18.)

Song, P., Singer, H.J. and Siscoe, H.L. (Eds.), 2001, Space Weather, vol. 125 of Geophysical Monograph, American Geophysical Union, Washington, DC (Cited on page 5.)

Living Reviews in Solar Physics

http: //www. livingreviews.org/lrsp-2006-2 
Srivastava, N. and Schwenn, R., 2000, "The origin of the solar wind: an overview", in The Outer Heliosphere: Beyond the Planets, (Eds.) Scherer, K., Fichtner, H., Marsch, E., Based on the spring school "Die äußere Heliosphäre - Jenseits der Planeten, in Bad Honnef, Germany, 12-16 April 1999, pp. 12-40, Copernicus, Katlenburg-Lindau (Cited on page 10.)

Srivastava, N. and Venkatakrishnan, P., 2002, "Relationship between CME Speed and Geomagnetic Storm Intensity", Geophys. Res. Lett., 29, 1. [DOI], [ADS] (Cited on page 50.)

Srivastava, N., Schwenn, R., Inhester, B., Stenborg, G. and Podlipnik, B., 1999a, "Measurements of Flow Speeds and Acceleration in Gradually Evolving Solar Mass Ejections as Observed by LASCO", in Solar Wind Nine, (Eds.) Suess, S.T., Gary, G.A., Nerney, S.F., Proceedings of the Ninth International Solar Wind Conference, Nantucket, Massachusetts, October, 1998, vol. 471 of AIP Conference Proceedings, p. 115, American Institute of Physics, Woodbury, NY. [ADS] (Cited on page 40.)

Srivastava, N., Schwenn, R., Inhester, B., Stenborg, G. and Podlipnik, B., 1999b, "Acceleration Profile of the Slow Solar Wind as Inferred from Gradual Mass Ejections Observed by LASCO", Space Sci. Rev., 87, 303-306. [DOI], [ADS] (Cited on page 40.)

Srivastava, N., Schwenn, R., Inhester, B., Martin, S.F. and Hanaoka, Y., 2000, "Factors Related to the Origin of a Gradual Coronal Mass Ejection Associated with an Eruptive Prominence on 1998 June 21-22", Astrophys. J., 534, 468-481. [DOI], [ADS] (Cited on page 48.)

St Cyr, O.C., Plunkett, S.P., Michels, D.J., Paswaters, S.E., Koomen, M.J., Simnett, G.M., Thompson, B.J., Gurman, J.B., Schwenn, R., Webb, D.F., Hildner, E. and Lamy, P.L., 2000, "Properties of coronal mass ejections: SOHO LASCO observations from January 1996 to June 1998", J. Geophys. Res., 105(A8), 18,169-18,186. [DOI], [ADS] (Cited on pages 39, 40, 44, and 50.)

Švestka, Z., 1976, Solar Flares, vol. 8 of Geophysics and Astrophysics Monographs, Reidel, Dordrecht; Boston; U.S.A. (Cited on page 21.)

Švestka, Z., 1981, "Flare observations", in Solar Flare Magnetohydrodynamics, (Ed.) Priest, E.R., vol. 1 of Fluid Mechanics of Astrophysics and Geophysics, pp. 47-137, Gordon and Breach, New York. [ADS], [Google Books] (Cited on pages 21 and 24.)

Švestka, Z., 2001, "Varieties of Coronal Mass Ejections and Their Relation to Flares", Space Sci. Rev., 95, 135-146. [DOI], [ADS] (Cited on pages 31 and 40.)

Thompson, B.J., 2000, "Moreton Waves", in Encyclopedia of Astronomy and Astrophysics, (Ed.) Murdin, P., p. 2575, Institute of Physics Publishing, Bristol. [ADS] (Cited on page 44.)

Thompson, B.J., Plunkett, S.P., Gurman, J.B., Newmark, J.S., St Cyr, O.C. and Michels, D.J., 1998, "SOHO/EIT observations of an Earth-directed coronal mass ejection on May 12, 1997", Geophys. Res. Lett., 25, 2465-2468. [DOI], [ADS] (Cited on page 44.)

Tripathi, D., Bothmer, V., Solanki, S.K., Schwenn, R., Mierla, M. and Stenborg, G., 2005, "SoHO/EIT Observation of a Coronal Inflow", in Coronal and Stellar Mass Ejections, (Eds.) Dere, K., Wang, J., Yan, Y., Proceedings of the IAU Symposium 226, held 13-17 September 2004, Beijing, China, pp. 133-134, Cambridge University Press, Cambridge; New York. [ADS] (Cited on page 44.)

Tsurutani, B.T. and Gonzalez, W.D., 1987, "The cause of high-intensity long-duration continuous AE activity (HILDCAAs): Interplanetary Alfvén wave trains", Planet. Space Sci., 35, 405-412. [DOI], [ADS] (Cited on page 18.) 
Tsurutani, B.T., Smith, E.J., Gonzalez, W.D., Tang, F. and Akasofu, S.I., 1988, "Origin of interplanetary southward magnetic fields responsible for major magnetic storms near solar maximum (1978-1979)", J. Geophys. Res., 93, 8519-8531. [DOI], [ADS] (Cited on pages 10, 47, and 48.)

Tsurutani, B.T., Gonzalez, W.D., Lakhina, G.S. and Alex, S., 2003, "The extreme magnetic storm of 1-2 September 1859", J. Geophys. Res., 108, 1. [DOI], [ADS] (Cited on page 21.)

Tsurutani, B.T., Gonzalez, W.D., Guarnieri, F.L., Kamide, Y., Zhou, X. and Arballo, J.K., 2004a, "Are high-intensity long-duration continuous AE activity (HILDCAA) events substorm expansion events?", J. Atmos. Sol.-Terr. Phys., 66, 167-176. [DOI], [ADS] (Cited on page 18.)

Tsurutani, B.T., Gonzalez, W.D., Zhou, X.-Y., Lepping, R.P. and Bothmer, V., 2004b, "Properties of slow magnetic clouds", J. Atmos. Sol.-Terr. Phys., 66, 147-151. [DOI], [ADS] (Cited on page 48.)

Tsurutani, B.T., Judge, D.L., Guarnieri, F.L., Gangopadhyay, P., Jones, A.R., Nuttall, J., Zambon, G.A., Didkovsky, L., Mannucci, A.J., Iijima, B., Meier, R.R., Immel, T.J., Woods, T.N., Prasad, S., Floyd, L., Huba, J., Solomon, S.C., Straus, P. and Viereck, R., 2005, "The October 28, 2003 extreme EUV solar flare and resultant extreme ionospheric effects: Comparison to other Halloween events and the Bastille Day event", Geophys. Res. Lett., 32, L03S09. [DOI], [ADS] (Cited on page 21.)

Tu, C.-Y. and Marsch, E., 1995, "MHD structures, waves and turbulence in the solar wind: Observations and theories", Space Sci. Rev., 73(1/2), 1-210. [DOI], [ADS] (Cited on page 17.)

Turner, R., 2001, "What we must know about solar particle events to reduces the risk to astronauts", in Space Weather, (Ed.) Song, P. et al., vol. 125 of Geophysical Monograph, pp. 39-44, American Geophysical Union, Washington, DC (Cited on page 32.)

Tylka, A.J., 2001, "New insights on solar energetic particles from Wind and ACE", J. Geophys. Res., 106(A11), 25,333-25,352. [DOI], [ADS] (Cited on page 32.)

Veselovsky, I.S., Panasyuk, M.I., Avdyushin, S.I., Bazilevskaya, G.A., Belov, A.V., Bogachev, S.A., Bogod, V.M., Bogomolov, A.V., Bothmer, V., Boyarchuk, K.A., Vashenyuk, E.V., Vlasov, V.I., Gnezdilov, A.A., Gorgutsa, R.V., Grechnev, V.V., Denisov, Y.I., Dmitriev, A.V., Dryer, M., Yermolaev, Y.I., Eroshenko, E.A., Zherebtsov, G.A., Zhitnik, I.A., Zhukov, A.N., Zastenker, G.N., Zelenyi, L.M., Zeldovich, M.A., Ivanov-Kholodnyi, G.S., Ignat'ev, A.P., Ishkov, V.N., Kolomiytsev, O.P., Krasheninnikov, I.A., Kudela, K., Kuzhevsky, B.M., Kuzin, S.V., Kuznetsov, V.D., Kuznetsov, S.N., Kurt, V.G., Lazutin, L.L., Leshchenko, L.N., Litvak, M.L., Logachev, Y.I., Lawrence, G., Markeev, A.K., Makhmutov, V.S., Mitrofanov, A.V., Mitrofanov, I.G., Morozov, O.V., Myagkova, I.N., Nusinov, A.A., Oparin, S.N., Panasenco, O.A., Pertsov, A.A., Petrukovich, A.A., Podorol'sky, A.N., Romashets, E.P., Svertilov, S.I., Svidsky, P.M., Svirzhevskaya, A.K., Svirzhevsky, N.S., Slemzin, V.A., Smith, Z., Sobel'man, I.I., Sobolev, D.E., Stozhkov, Y.I., Suvorova, A.V., Sukhodrev, N.K., Tindo, I.P., Tokhchukova, S.K., Fomichev, V.V., Chashey, I.V., Chertok, I.M., Shishov, V.I., Yushkov, B.Y., Yakovchouk, O.S. and Yanke, V.G., 2004, "Solar and Heliospheric Phenomena in October-November 2003: Causes and Effects", Cosmic Res., 42, 435-488. [ADS] (Cited on page 7.)

Vourlidas, A., Buzasi, D., Howard, R.A. and Esfandiari, E., 2002, "Mass and energy properties of LASCO CMEs", in Solar Variability: From Core to Outer Frontiers, (Ed.) Wilson, A., Proceedings of the 10th European Solar Physics Meeting, Prague, Czech Republic, 9-14 September 2002, vol. SP-506 of ESA Conference Proceedings, pp. 91-94, ESA, Noordwijk. [ADS] (Cited on page 40.)

Living Reviews in Solar Physics

http://www.livingreviews.org/lrsp-2006-2 
Vršnak, B., Magdalenić, J. and Zlobec, P., 2004, "Band-splitting of coronal and interplanetary type II bursts. III. Physical conditions in the upper corona and interplanetary space", Astron. Astrophys., 413, 753-763. [DOI], [ADS] (Cited on page 37.)

Wagner, W.J., 1984, "Coronal Mass Ejections", Annu. Rev. Astron. Astrophys., 22, 267-289. [DOI], [ADS] (Cited on page 39.)

Wagner, W.J. and MacQueen, R.M., 1983, "The excitation of type II radio bursts in the corona", Astron. Astrophys., 120, 136-138. [ADS] (Cited on page 29.)

Wang, Y.-M., Sheeley Jr, N.R., Howard, R.A., St Cyr, O.C. and Simnett, G.M., 1999, "Coronagraph observations of inflows during high solar activity", Geophys. Res. Lett., 26, 1203-1206. [DOI], [ADS] (Cited on page 44.)

Warmuth, A. and Mann, G., 2005, "A model of the Alfvén speed in the solar corona", Astron. Astrophys., 435, 1123-1135. [DOI], [ADS] (Cited on page 37.)

Webb, D.F. and Howard, R.A., 1994, "The solar cycle variation of coronal mass ejections and the solar wind mass flux", J. Geophys. Res., 99(A3), 4201-4220. [DOI], [ADS] (Cited on page 50.)

Webb, D.F., Cliver, E.W., Gopalswamy, N., Hudson, H.S. and St Cyr, O.C., 1998, "The solar origin of the January 1997 coronal mass ejection, magnetic cloud and geomagnetic storm", Geophys. Res. Lett., 25, 2469-2472. [DOI], [ADS] (Cited on page 50.)

Webb, D.F., Crooker, N.U., Plunkett, S.P. and St Cyr, O.C., 2001, "SEPs: Space Weather Hazard in Interplanetary Space", in Space Weather, (Ed.) Song, P. et al., vol. 125 of Geophysical Monograph, pp. 123-141, American Geophysical Union, Washington, DC (Cited on page 39.)

Wei, F., Liu, R., Fan, Q. and Feng, X., 2003, "Identification of the magnetic cloud boundary layers", J. Geophys. Res., 108(A6), 1263. [DOI], [ADS] (Cited on page 47.)

Wild, J.P., 1950, "Observations of the Spectrum of High-Intensity Solar Radiation at Metre Wavelengths. III. Isolated Bursts", Aust. J. Sci. Res. A, 3, 541. [ADS] (Cited on page 25.)

Wilson, J.W., Clowdsley, M.S., Cucinotta, F.A., Tripathi, R.K., Nealy, J.E. and de Angelis, G., 2004, "Deep space environments for human exploration", Adv. Space Res., 34, 1281-1287. [DOI], [ADS] (Cited on page 7.)

Woods, T.N., Eparvier, F.G., Fontenla, J., Harder, J., Kopp, G., McClintock, W.E., Rottman, G., Smiley, B. and Snow, M., 2004, "Solar irradiance variability during the October 2003 solar storm period", Geophys. Res. Lett., 31, L10802. [DOI], [ADS] (Cited on page 7.)

Yashiro, S., Gopalswamy, N., Michalek, G., St Cyr, O.C., Plunkett, S.P., Rich, N.B. and Howard, R.A., 2004, "A catalog of white light coronal mass ejections observed by the SOHO spacecraft", J. Geophys. Res., 109, 7105. [DOI], [ADS] (Cited on pages 40 and 43.)

Yurchyshyn, V., Wang, H. and Abramenko, V., 2004, "Correlation between speeds of coronal mass ejections and the intensity of geomagnetic storms", Space Weather, 2, S02001. [DOI], [ADS] (Cited on page 50.)

Yurchyshyn, V.B., Wang, H., Goode, P.R. and Deng, Y., 2001, "Orientation of the Magnetic Fields in Interplanetary Flux Ropes and Solar Filaments", Astrophys. J., 563, 381-388. [DOI], [ADS] (Cited on page 48.)

Zhang, J., Dere, K. and Howard, R.A., 2001, "Relationship Between Coronal Mass Ejections and Flares", Eos Trans. AGU, 82, 47. [ADS] (Cited on page 44.) 
Zhang, J., Dere, K.P., Howard, R.A. and Vourlidas, A., 2004, "A Study of the Kinematic Evolution of Coronal Mass Ejections", Astrophys. J., 604, 420-432. [DOI], [ADS] (Cited on page 44.)

Zhao, X.P. and Hoeksema, J.T., 1997, "Is the geoeffectiveness of the 6 January 1997 CME predictable from solar observations?", Geophys. Res. Lett., 24, 2965-2968. [DOI], [ADS] (Cited on page 50.)

Zhao, X.P., Hoeksema, J.T. and Marubashi, K., 2001, "Magnetic cloud $B_{s}$ events and their dependence on cloud parameters", J. Geophys. Res., 106, 15643-15656. [DOI], [ADS] (Cited on page 48.)

Zirker, J.B. (Ed.), 1977, Coronal Holes and High-Speed Wind Streams, A Monograph from Skylab Solar Workshop I, Colorado Associated University Press, Boulder (Cited on page 10.) 